\title{
Lapita to Post-Lapita transition: Insights from the chemical analysis of pottery from the sites of Teouma, Mangaasi, Vao and Chachara, Vanuatu
}

\author{
Mathieu Leclerc
}

\begin{abstract}
Lapita and Post-Lapita ceramic collections from four archaeological sites scattered across two of the main islands of Vanuatu were characterised using LA-ICP-MS. Results from the analysis of 112 ceramic samples show that the decorated ceramics from Lapita sites are generally more compositionally variable than the later assemblages. Not only do the early sites contain more exotic samples, but the early locally made decorated vessels also display a wider compositional range. This is interpreted as revealing behaviours of potters initially settling into new territories before developing recurrent habits in terms of pottery manufacture. The decrease in variability of the technological styles encountered between Lapita and Post-Lapita occupations suggest that important social modifications occurred: increasingly sedentary populations, changes in the social structure and political economy, and the collapse of the symbolic Lapita belief system and its ceremonial practices.
\end{abstract}

\section{Introduction}

Lapita pottery is generally characterised by greater decorative, morphological and technological variability compared to pottery associated with subsequent occupations. In terms of decoration, there is a gradual transformation from complex and intricate dentate-stamped motifs to coarser and more open decorations, as seen for example in the Arawes (Summerhayes 2000a) and Mussau (Kirch et al. 1991:151) in the Bismarcks. A similar trend has also been observed regarding the range of vessel forms that decreases over time. While a variety of flat dishes, cylinder stands and other carinated vessels have been recovered from Lapita deposits, Post-Lapita occupations display collections largely dominated by globular vessels (Bedford 2006b; Kirch 2000; Summerhayes $2000 \mathrm{~b})$. The range of raw materials used to manufacture ceramics and their compositional diversity decreases over time, as observable for example at Watom (Anson 1999; Green and Anson 1991) and Mussau (Hunt 1989:209). 
The data set presented here results from a broad program of chemical characterisation of Lapita and Post-Lapita ceramics from Vanuatu (Leclerc 2016). The results from the analysis by laser ablation inductively coupled plasma mass spectrometry (LA-ICP-MS) of ceramic samples come from four archaeological sites (Teouma, Mangaasi, Vao and Chachara) scattered across two of the main islands of Vanuatu (Efate and Malakula) (Figure 17.1). Given the composition of the collections and the well-established chronology of the sites in question, the results provide an opportunity to verify if this general trend of simplification of assemblages over time also occurred in Vanuatu, as has been suggested by Bedford (2006b) and Dickinson et al. (2013). The data set presented in this chapter, along with its implications in terms of social organisation and behaviour, complement earlier contributions and allows us to gain further insights into the sociocultural changes between Lapita and Post-Lapita groups. The focus here is to identify any diachronic changes in pottery technological styles. The decorated Lapita pottery assemblage will be compared to the Post-Lapita collection from each island in order to understand how the selection processes of raw materials changed through time. This allows verification of whether synchronised modification of decorative and technological aspects of pottery production occurred at the end of the Lapita period.
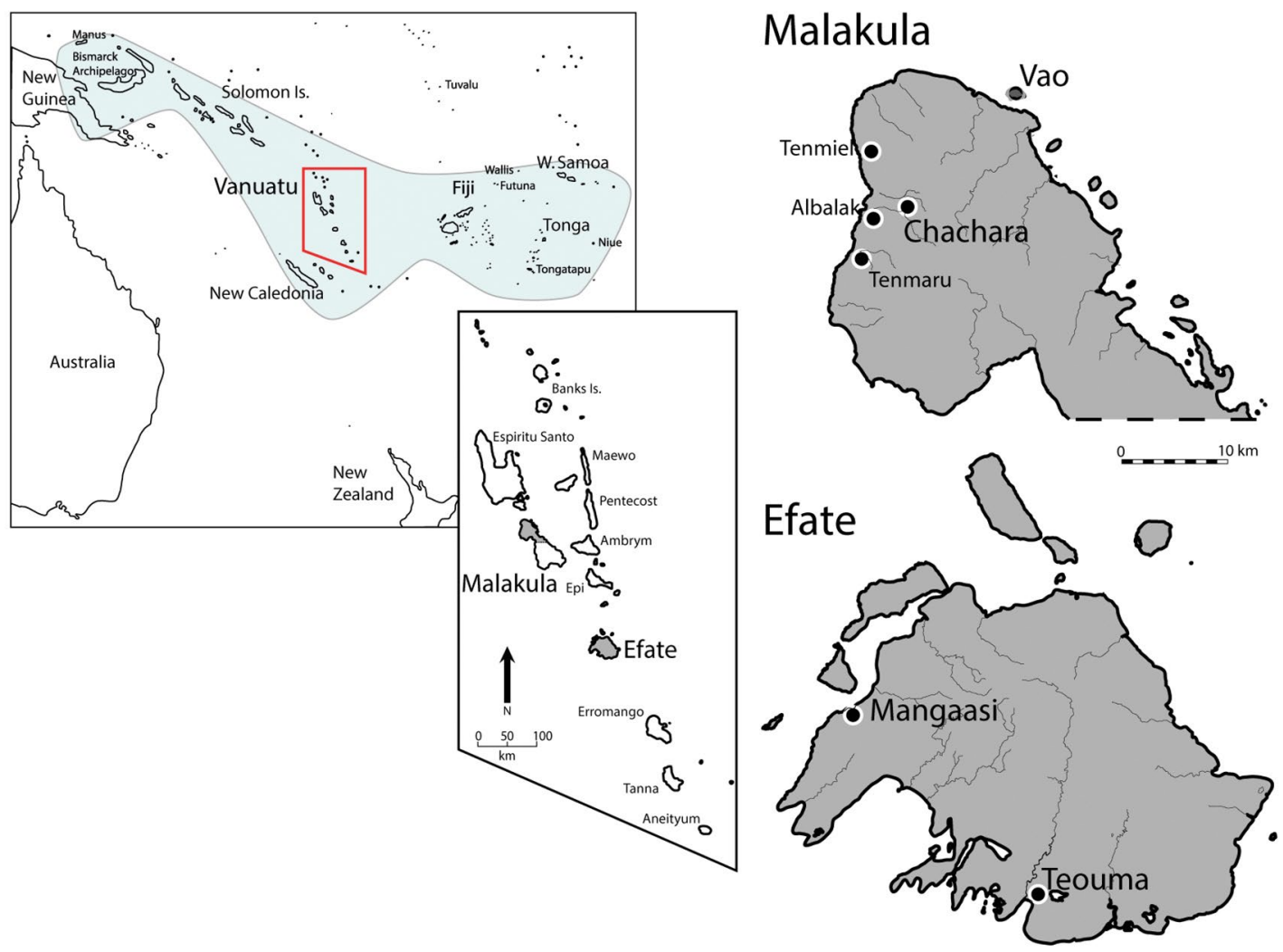

Figure 17.1. Localisation of Vanuatu and of the islands of Efate and Malakula in particular, with relevant archaeological sites identified.

Source: Illustration by author. 


\section{Technological style}

During the course of pottery manufacturing, as is the case for any object produced, many technological choices have to be taken by the potter in order to reach their goal and produce a ceramic vessel that is satisfying in every aspect (performance, appearance, economic and symbolic role, etc.). These behaviours are adopted and chosen among a vast array of equifinal possibilities based on choices that are meaningful socially, economically and ideologically (Lechtman 1977; Rye 1976; Sillar and Tite 2000). Technology can thus be considered as 'the materialisation of social thought' (Dobres and Hoffman 1994:221). If we consider 'style' as the manifest impression of cultural patterning (Lechtman 1977:4), or in other words as the part of formal variability in material culture that is culturally significant, active and conveying information (Conkey 1978; Wobst 1977:321), then technology has a style of its own. This technological style comprises the stylistic elements embedded into its technological features and is the expression, on the level of technological behaviour, of underlying cultural values, patterns, codes, standards and rules (Hegmon 1998:268; Lechtman 1977:13; Lemonnier 1993). The investigation of the technological style of an object thereby allows the interpretation of its traits in terms of behaviours. Assuming the tenet that style has an active messaging role, change in technological style suggests that important changes are happening elsewhere in society as well (Conkey 1978; Peacock 1970:375; Wobst 1977).

\section{Social organisation and Lapita pottery}

In order to interpret satisfactorily the data presented here, it is necessary to briefly review the main hypotheses relating to the nature of the Lapita Cultural Complex, and most importantly the significance given to the dentate-stamped vessels. It is crucial to have an idea of the role played by Lapita pots, and what they represented for the people using them, to understand the significance of any changes that occurred through time in terms of decorative and, most relevant for this study, technological features.

The recurrent set of archaeological attributes associated with the first recorded human presence in Remote Oceania is gathered under the encompassing concept named the Lapita Cultural Complex (Green 1991, 2000; Kirch 1997; Nunn and Petchey 2013; Sand 2010; Sand and Bedford 2010; Sand et al. 2011; Sheppard 2011; Sheppard et al. 2015; Specht et al. 2014). The full geographic extent of Lapita occupation is found across both Near and Remote Oceania, stretching from the south coast of New Guinea in the west (Skelly et al. 2014) to Tonga and Samoa in the east (Kirch 1997). The most distinctive element of the Lapita Cultural Complex is its decorated pottery, characterised by fine dentate-stamped designs and representing the earliest evidence of pottery-making in the region (Green 1991, 2000).

It was previously thought that the Lapita exchange network was an essential component of the strategy of expansion and acted as a lifeline linking the new colonies to their homeland communities by maintaining long-distance exchange routes between remote localities (Green 1987:246; Green and Kirch 1997:28-30; Kirch 1988, 1991; Sheppard 1993). However, Lapita pottery assemblages from Vanuatu (Dickinson et al. 2013; Leclerc 2016), the Arawes (Summerhayes 2000a), New Caledonia (Chiu 2003a:176; Galipaud 1990), Watom (Green and Anson 1991) and Tonga (Dickinson 2006a; Dickinson et al. 1996) have since revealed that most Lapita pots were manufactured and/or exchanged locally rather than travelling long distances. Consequently, there has probably never been a single integrated 'Lapita exchange network' that spanned the entire geographic range over which Lapita sites are distributed. 
Considering that very few long-distance exchanges occurred, it is surprising that the recurrent presence of decorative motifs, organised following common culturally encoded rules (Kirch 2000:102; Mead 1975; Sand 2007; Siorat 1990), is found over a region covering $4000 \mathrm{~km}$, albeit with some regional variation (Chiu 2007; Sand 2007; Spriggs 1990, 2002). This demonstrates that ideas rather than objects were being exchanged (Earle and Spriggs 2015). This implies that the pots held social roles and that the decorations conveyed a message comprehensible to the people who were manufacturing and using them (Ambrose 1997:530). The recent analysis of organic residue absorbed in Lapita pottery from Teouma (Vanuatu) also supports this idea that dentate-stamped pots held a significance for associated communities (Leclerc et al. 2018).

The main consensus is that dentate-stamped Lapita vessels had a function related to the ceremonial sphere of activities (Kirch 2000:102-106). Variations on the theme have been suggested by many: it was proposed that Lapita could represent 'culture elements in the material paraphernalia of some kind of cult, dance complex or social ritual' (Terrell and Welsch 1997:568). Similarly, Best (2002:99-100) suggested that decorated Lapita pots could be paraphernalia associated with religious activities and a manifestation of the concentration and consolidation of power within an emerging social and religious complex. Spriggs (2003:205) had the pots as symbols holding an important role in the 'ritual performance' of 'a new ethnic identity ... forged around a new prestige language (now labelled Proto-Oceanic Austronesian), which was spoken by people of originally disparate geographical and genetic origins'. Chiu (2005:6; 2007:245, 257-260) put forward the idea that dentate-stamped pots were symbols representing social identities within the hierarchical structure of ethnically mixed Lapita 'House societies'. More generally, they have been seen as 'representations of ancestors ... functioning within a ritual system or cult of ancestors, as well as constituting objects of reciprocal exchange among kinship groups' (Kirch 2000:104-105). All in all, it is generally accepted that:

whatever the reason, it would appear that it [the Lapita design system] materialized something fundamental to Lapita society that needed to be reproduced by small colonizing populations [and that] the pots were actively involved in some form of ongoing system. (Sheppard 2011:804)

As Lapita dispersal progressed, wider networks gradually disintegrated and localised exchange networks developed (Bedford and Clark 2001; Kirch 1997:70). A regional fragmentation process developed and the shared Lapita system, whatever it constituted, gradually lost its relevance. Ongoing social transformation spanning a few generations was occurring and dentate-stamped pottery was significantly modified before eventually ceasing to be produced (Best 2002; Summerhayes 2000a:232). Other types of decoration such as incision, impression and applied relief became more dominant in assemblages Post-Lapita.

\section{Archaeological sites-Efate}

\section{Teouma}

The Lapita site at Teouma is located at about $8 \mathrm{~m}$ above current sea level at some $800 \mathrm{~m}$ from the south coast of Efate and Teouma Bay. Archaeological deposits of Lapita age were well preserved, buried beneath up to $80 \mathrm{~cm}$ of black tephra-rich sediment (Bedford et al. 2010:141). Initial site use was as a cemetery and an adjacent contemporaneous settlement (Bedford et al. 2010:143145). The use of the site as a cemetery was relatively short-term, as suggested by contextual and chronological markers (Bedford et al. 2010:145; Valentin et al. 2014). The earliest signs of activity are dated to c. 2970 cal. BP with regular use of the Lapita cemetery from 2940 to 2710 cal. BP (Petchey et al. 2014; Petchey et al. 2015). These dates, along with the presence 
of West New Britain obsidian (Constantine et al. 2015; Reepmeyer et al. 2010), a range of extinct fauna (White et al. 2010; Worthy et al. 2015) and the diversity of pottery vessels and motifs indicate that it is a colonising site on Efate (Bedford et al. 2010; cf. Sheppard 1993).

The Teouma ceramic collection is remarkable in its variability in terms of forms and motifs, especially in the cemetery area (Bedford and Spriggs 2007; Bedford et al. 2006; Bedford et al. 2007; Bedford et al. 2010:145-147). The dentate-stamped ceramic collection is dominated by various forms of carinated vessels, but also comprises flat dishes, cylinder stands and other globular and outcurving vessels. Dentate stamping dominates the assemblage, although recent analysis has revealed that incised vessels represent a contemporaneous and more important component of decoration than previously thought (Spriggs and Bedford 2013). A wide array of geometric, curvilinear and anthropomorphic decorative motifs have been applied on Teouma vessels (Bedford et al. 2010:147; Bedford et al. 2007), but plain pots are also included in the Lapita assemblage. A midden layer representing immediately Post-Lapita occupation and including pottery displaying Arapus (c. 2800 cal. BP) and Early Erueti (c. 2800-2500 cal. BP) styles sits above the cemetery layer (Bedford et al. 2010:145). The plurality of Lapita forms and decorative techniques disappears through the midden layer in favour of a hegemonic globular outcurving rim vessel (Bedford et al. 2010:147).

In terms of temper, the petrographic analysis of the Teouma dentate-stamped collection revealed that five local variants of a 'standard Efate temper', differentiated one from another by different proportions of their main minerals (i.e. plagioclase, clinopyroxene, opaque iron oxides, volcanic rock fragments and calcareous grains), represented the vast majority of the analysed collection (100 pots, 89 per cent). The variable concentrations of heavy and light minerals were interpreted to reflect various degrees of reworking, indicating their origin from stream or beach. The remaining part of the assemblage (12 pots, 11 per cent) contained sands exotic to Efate. The exotic nature of these samples was demonstrated by the presence of diagnostic minerals in their temper (e.g. hornblende, quartz, glaucophane), revealing their origins from further north in Vanuatu and in some cases from New Caledonia (Dickinson et al. 2013).

\section{Mangaasi}

The Mangaasi site, along with the contiguous Arapus site, is located on the north-west coast of Efate facing the offshore islands of Lelepa and Retoka. Their excavation yielded a large quantity of plain and decorated sherds, the latter displaying decoration of incised and applied bands (Bedford 2006b; Bedford and Spriggs 2000; Garanger 1971; Spriggs and Bedford 2001). The presence of tephra-rich layers and a clear vertical and horizontal stratigraphy helped establish the chronology of the site (occupied between $2800 \mathrm{BP}$ and approximately $1200 \mathrm{BP}$ ) and contributed to the understanding of the Post-Lapita ceramic sequence for the region. Successive ceramic styles identified and defined included Arapus (from c. 2800 BP), Erueti (from c. 2800$2700 \mathrm{cal} . \mathrm{BP}$ ) and Mangaasi (from c. $2200 \mathrm{cal}$. BP), each characterised by specific decorative and morphological traits (Bedford 2006b).

Overall, the Mangaasi ceramic collection is characterised by great homogeneity in terms of temper and fabrics: 'the ceramics recovered from Mangaasi ... show no significant change in their mineral composition over time' (Bedford 2006b:109). The petrographic examination of sherds from the Garanger excavation at Mangaasi and the surrounding offshore islets of Lelepa, Mele and Retoka revealed that their tempers were composed mainly of plagioclase feldspar grains with subordinate rock fragments composed of brown volcanic glass fragments and minor amounts of clinopyroxene and opaque iron oxides (Dickinson 1995:4-5). This description corresponds with Efate stream sands derived from pumice (Dickinson 1995:5). Two additional Erueti-style sherds recovered during more recent excavations in a layer well below the depth of excavation 
reached by Garanger displayed the same combination of minerals corresponding with the typical Efate temper type (Dickinson 2006b). In contrast to Teouma, the later sherds from Efate were produced locally, which was also supported by the recovery of 26 wasters from the Mangaasi site during the 1996-1999 excavations (Bedford 2006b:108).

\section{Archaeological sites-Malakula}

\section{Vao}

The Lapita site on Vao Island is located on the sheltered west side of the island on an uplifted back beach terrace facing the mainland of Malakula (Bedford et al. 2011:28). Lapita sites were recorded on four of the small islands off the north-east coast of Malakula and excavations on Vao revealed a well-preserved Lapita midden with material sealed beneath a heavily compacted layer made of locally imported worn branch coral, pebbles and tephra-laden soil (Bedford 2007:188; Bedford et al. 2011:28). The initial Lapita settlements on the small islands seem to have lasted from 2900 to $2600 \mathrm{BP}$ (Bedford et al. 2011:34) and the initial settlement of Vao occurred slightly earlier than on the other islands (Bedford et al. 2011; Bedford and Galipaud 2010:127). The range of vessel forms represented at Vao includes carinated vessels, shallow and deeper incurving bowls and a single flat-bottomed dish (see Bedford this volume). Globular plainware vessels with outcurving rims were also found in association, and several incised sherds have been recorded (Bedford 2007:189, 2003:154). Interestingly, the assemblage also revealed evidence showing that red and white painting had been applied on dentate-decorated Lapita sherds after firing (Bedford 2006a). Three Lapita and four Post-Lapita burials were also recorded, none of them included any grave goods (Bedford et al. 2011:31-32). The sherds recovered from the more recent upper layers differ from those associated with the Lapita occupation and display characteristics similar to the recent pottery found ubiquitously on the surface of Malakula.

In terms of temper, examination of 20 of these Post-Lapita sherds revealed volcanic tempers composed principally of felsitic volcanic rock fragments together with plagioclase, clinopyroxene, hornblende and opaque iron oxides. Such a volcanic detritus set corresponds with the Lower to Middle Miocene bedrock assemblage of Malakula (Dickinson 1995:4). A further set of 22 Lapita sherds from Vao was also examined by Dickinson (2003). With the exception of one sample, every sherd had a mineralogical content corresponding to a 'local' origin from Malakula or nearby Santo. The vast majority displayed texturally varied, albeit generically related, non-placer lithic-rich volcanic sand tempers that almost certainly derive from inland stream sources on Malakula. Occasionally the tempers comprised calcareous grains. The occurrence of terrigenous tempers on Vao suggests that the raw materials used and/or the vessels produced were imported from mainland Malakula or further afield since these kinds of deposits cannot be found on Vao Island itself.

The only definitively exotic sample from the Vao Lapita collection differs from the rest of the group by a very high content of volcanic glass fragments probably from reworked volcanic ash of stream origin. While large quantities of glass fragments are typically associated with Efate and Shepherds tempers, the relative occurrence is so high in this case that its origin is most likely the Paama-Lopevi-Epi cluster of islands to the south-east of Malakula (Dickinson 2003:3). Interestingly, this exotic sherd does not appear distinctive macroscopically nor in terms of decoration (Dickinson 2003:5). 


\section{Chachara}

Lastly, the excavation of the Chachara site, located about $2.3 \mathrm{~km}$ inland from the north-west coast of Malakula, revealed a layer of concentrated midden material comprising pottery, shellfish, bone and a shell adze (Bedford 2006b:66). The ceramic assemblage is homogenous, both in vessel form and in fabric, and displayed characteristics corresponding to the late period ceramics found across much of Malakula, which consist of two variants of coil-made tubular unrestricted vessels ('bullet-shaped' pots) differentiated by their respective surface treatment and decoration. 'The homogenous nature of the ceramics at the site suggest a relatively short-term period of occupation dating to sometime around 654-489 BP' (Bedford 2006b:67).

Ten stylistically varied late-style sherds from various sites on Malakula, including three from Chachara, were analysed petrographically by Dickinson (2006c). The texture and composition of the tempers differ from one sherd to another, but they all contain stream sands derived from Miocene volcanic bedrock units from the island interior (Mitchell 1966, 1971). Different proportions of pyroxene and hornblende content as well as variation in internal structure of volcanic rock fragments differentiate the tempers one from another. As opposed to earlier Lapita sherds, none of them contain calcareous grains. Even with these slight variations between types, the overall similarity of the temper sands suggests that similar temper sands were available in many widely distanced locales of the island. One unusual surface sherd from Tenmaru, however, revealed a temper displaying characteristics matching Santo temper (Dickinson 2006d).

\section{Method}

\section{Sampling}

A total of 112 ceramic samples recovered from these archaeological sites were analysed (Figure 17.2 shows a subset of the samples ${ }^{1}$ ). For sites on Efate, 26 dentate-stamped sherdsrepresenting 23 different vessels from Teouma-and 33 samples from Mangaasi-4 wasters and 29 sherds representative of the variability encountered in terms of decorative style (10 Erueti, 6 Late Erueti, 9 Mangaasi and 4 Late Mangaasi)—were analysed. From Vao, 31 samples were analysed, 28 dentate-stamped, two incised (va40 and va44) and one with applied relief decoration (va43). One of the incised samples was recovered from the Lapita layer (va40) while the other incised (va44) and the applied relief (va43) samples are associated with horizons that are more recent. Lastly, 22 samples from various Post-Lapita sites across the north-west coast of Malakula (17 from Chachara, three from Tenmiel, and a single piece each from Tenmaru and Albalak) were also analysed.

The selected samples had to be generally well-dated and displaying decoration and/or vessel forms related to a phase of their regional sequence. The selection of decorated large-sized sherds was thus prioritised since it provided the opportunity to relate the compositional results with the controlled chronological and geographical context of the regional ceramic sequences. Selected sherds had also to be large enough to produce samples of sufficient size ( 2 by $2 \mathrm{~cm}$ ) to be deemed representative of the internal compositional variability of the sherd-vessel. An effort was also made to select samples representative of each dominant paste recipe identified petrographically, in order to cover the entire range of variability encountered.

1 The complete set of pictures and contextual details for the samples are in Leclerc (2016). 

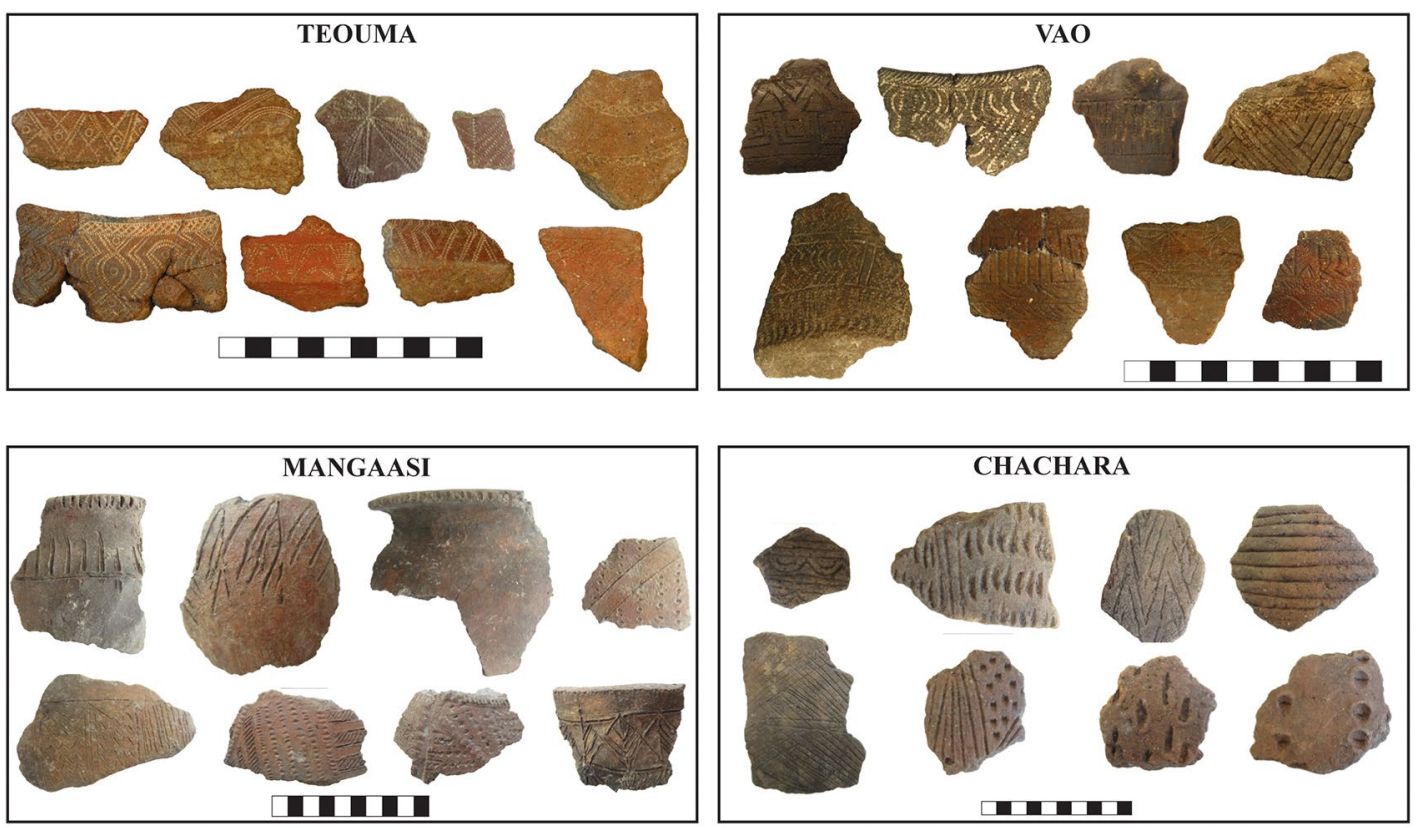

Figure 17.2. Subset of the sherd samples analysed.

Source: Illustration by author.

\section{LA-ICP-MS analysis}

Sample preparation and analytical procedures are described at length elsewhere (Leclerc 2016; Leclerc et al. 2019) so only the major points are emphasised here. Samples were analysed using a LA-ICP-MS instrument hosted at the Research School of Earth Sciences at The Australian National University. Prior to the analysis, the external surfaces were abraded to remove any potential surface contaminants and additional clay applied as a slip. The samples were then powdered in an agate mortar and pressed into pellets.

It was decided to analyse homogenised samples rather than separate the clay matrix from the inclusions because it is more suitable to address the research questions presented. It is true that analysing separately the clay matrix and the inclusions can ease the comparison of ceramic samples with clay deposits in certain circumstances (e.g. Ambrose 1992; Gaffney et al. 2015; Summerhayes 1997, 2000a). In this instance, however, the aim was not so much to identify the provenance of the raw materials but rather to describe as accurately as possible the components and the variability within the assemblages. Even if the former intention had been the case, the documented compositional variability of most natural clay deposits in Vanuatu would be prone to lead to mistaken associations between the clay fraction of a sherd and specific procurement areas (Leclerc 2016). In addition, the process of separating the components disentangles the constituents carrying information associated with natural and behavioural parameters. If the investigation undertaken aims to understand the decisions that resulted in the production of the object, as is the case here, then it appears preferable to consider the pottery as a behavioural unit rather than limiting the analysis to some selected aspects based on current analytical mindsets. To separate clays from inclusions results in the analysis of something that did not really exist in the past or at least in the potter's mind. Lastly, it can be difficult to analyse exclusively the clay matrix of some sherds depending on their fabric. To differentiate between manually added inclusions from those naturally present in the clay deposits can become a relatively subjective process that depends mostly on the competencies of the analyst, the representativeness of the area of the sample investigated and assumptions about the activities of the potter. Under these conditions, it is not guaranteed that the clay fraction of a sample will be comparable with clay 
deposits, making such separation of little use. In the context of Vanuatu at least, it is therefore argued that it is preferable to undertake compositional bulk analysis in order to understand the cultural motivations behind the technological decisions.

A set of five reference materials (SRM610, SRM612, SRM679, ANU2000 and NORC) was analysed twice during each analytical run. Out of the 45 isotopes analysed, a set of 40 yielded data of sufficient quality (Table 17.1). Counts obtained from the spectrometer were converted into concentrations using the method presented first in Gratuze (1999) and later detailed and used in many projects involving LA-ICP-MS analysis of pottery (Golitko et al. 2012; Gratuze et al. 2001; Neff 2003; Speakman and Neff 2005).

Principal component analysis (PCA) was applied to the data in order to extract patterns using GenStat 14.2 and JMP 12 (Harding and Payne 2011; SAS Institute Inc. 2015). The way PCA was undertaken was aimed at producing interpretable components that could be related to specific elements (Leclerc 2016:90-95). Five principal components were thus extracted from 18 relevant elements, each of them reproducing significantly the variance for a specific set of elements (Table 17.2). An alternative PCA excluding the elements prone to post-depositional alteration (particularly calcium, potassium and sodium (Buxeda i Garrigós et al. 2002; Picon 1991)) was undertaken to guarantee that the conclusions were not caused by differential weathering. The very similar results obtained by the two PCA confirm that the variability observed does not result from weathering processes.

Table 17.1. List of isotopes targeted during the LA-ICP-MS analysis with the 18 elements involved in the PCA highlighted.

\begin{tabular}{|l|l|l|l|l|l|l|l|}
\hline${ }^{7} \mathrm{Li}$ & ${ }^{11} \mathrm{~B}$ & ${ }^{23} \mathrm{Na}$ & ${ }^{24} \mathrm{Mg}$ & ${ }^{27} \mathrm{Al}$ & ${ }^{29} \mathrm{Si}$ & ${ }^{39} \mathrm{~K}$ & ${ }^{43} \mathrm{Ca}$ \\
\hline${ }^{47} \mathrm{Ti}$ & ${ }^{51} \mathrm{~V}$ & ${ }^{55} \mathrm{Mn}$ & ${ }^{57} \mathrm{Fe}$ & ${ }^{59} \mathrm{Co}$ & ${ }^{60} \mathrm{Ni}$ & ${ }^{65} \mathrm{Cu}$ & ${ }^{66} \mathrm{Zn}$ \\
\hline${ }^{71} \mathrm{Ga}$ & ${ }^{72} \mathrm{Ge}$ & ${ }^{75} \mathrm{As}$ & ${ }^{85} \mathrm{Rb}$ & ${ }^{88} \mathrm{~S} r$ & ${ }^{89} \mathrm{Y}$ & ${ }^{90} \mathrm{Zr}$ & ${ }^{93} \mathrm{Nb}$ \\
\hline${ }^{95} \mathrm{Mo}$ & ${ }^{118} \mathrm{~S}$ & ${ }^{133} \mathrm{CS}$ & ${ }^{137} \mathrm{Ba}$ & ${ }^{139} \mathrm{La}$ & ${ }^{140} \mathrm{Ce}$ & ${ }^{146} \mathrm{Nd}$ & ${ }^{147} \mathrm{Sm}$ \\
\hline${ }^{157} \mathrm{Gd}$ & ${ }^{163} \mathrm{Dy}$ & ${ }^{166} \mathrm{Er}$ & ${ }^{172} \mathrm{Yb}$ & ${ }^{178} \mathrm{Hf}$ & ${ }^{208} \mathrm{~Pb}$ & ${ }^{232} \mathrm{Th}$ & ${ }^{238} \mathrm{U}$ \\
\hline
\end{tabular}

Source: Author's summary.

Table 17.2. Set of elements significantly loading on each principal component extracted.

\begin{tabular}{|l|c|c|}
\hline Component & Variables loading significantly on the component & Proportion of total variance explained (total) \\
\hline 1 & $\mathrm{Na}, \mathrm{K}, \mathrm{Rb}$ & $28.3 \%(28.3 \%)$ \\
\hline 2 & $\mathrm{Nb}, \mathrm{Zr}, \mathrm{Sn}, \mathrm{Hf}, \mathrm{Pb}, \mathrm{Th}$ & $17.0 \%(45.4 \%)$ \\
\hline 3 & $\mathrm{Ti}, \mathrm{V}, \mathrm{Fe}, \mathrm{Co}$ & $15.9 \%(61.3 \%)$ \\
\hline 4 & $\mathrm{Y}, \mathrm{Nd}$ (REY) & $12.6 \%(73.8 \%)$ \\
\hline 5 & $\mathrm{Al}, \mathrm{Ca}, \mathrm{Sr}$ & $12.4 \%(86.2 \%)$ \\
\hline
\end{tabular}

Source: Author's summary.

\section{Results}

For the vast majority of elements, the variability encountered within the Teouma decorated ceramic collection was much more significant than the variability of Mangaasi ceramics, as illustrated by Figure 17.3 and Table 17.3 (see Table 17.4 for complete results). In fact, the Teouma assemblage had the highest variability of all the sites included in this study. These results correspond with petrographic data, suggesting that the people who lived at Teouma had a range of pottery vessels manufactured using a wide variety of tempers. Even though the detailed comparison of LA-ICP-MS data and petrographic temper types for each site is beyond 
the scope of this chapter, ${ }^{2}$ it is worth noting two things. First, the compositional data show that samples with the same petrographic temper types, and thus with similar proportions of the same minerals, are compositionally variable (see the distribution of the samples with unplacered and opaque-rich tempers in Figure 17.3 for example). The calcareous tempered vessels of Teouma also show great compositional dissimilarity particularly for PC2, PC4 and PC5, which indicates that their differential compositions do not originate principally from their different proportions of calcareous grains (Figure 17.3). This suggests that the variability originates from the clay matrix. Second, both petrographic and compositional data sets generally correspond in terms of distribution and groupings, as would be expected given the documented influence of inclusions on bulk chemical compositions of homogenised ceramic samples (Arnold et al. 1999; Chiu 2003b; Summerhayes 1997:115, 2000a).

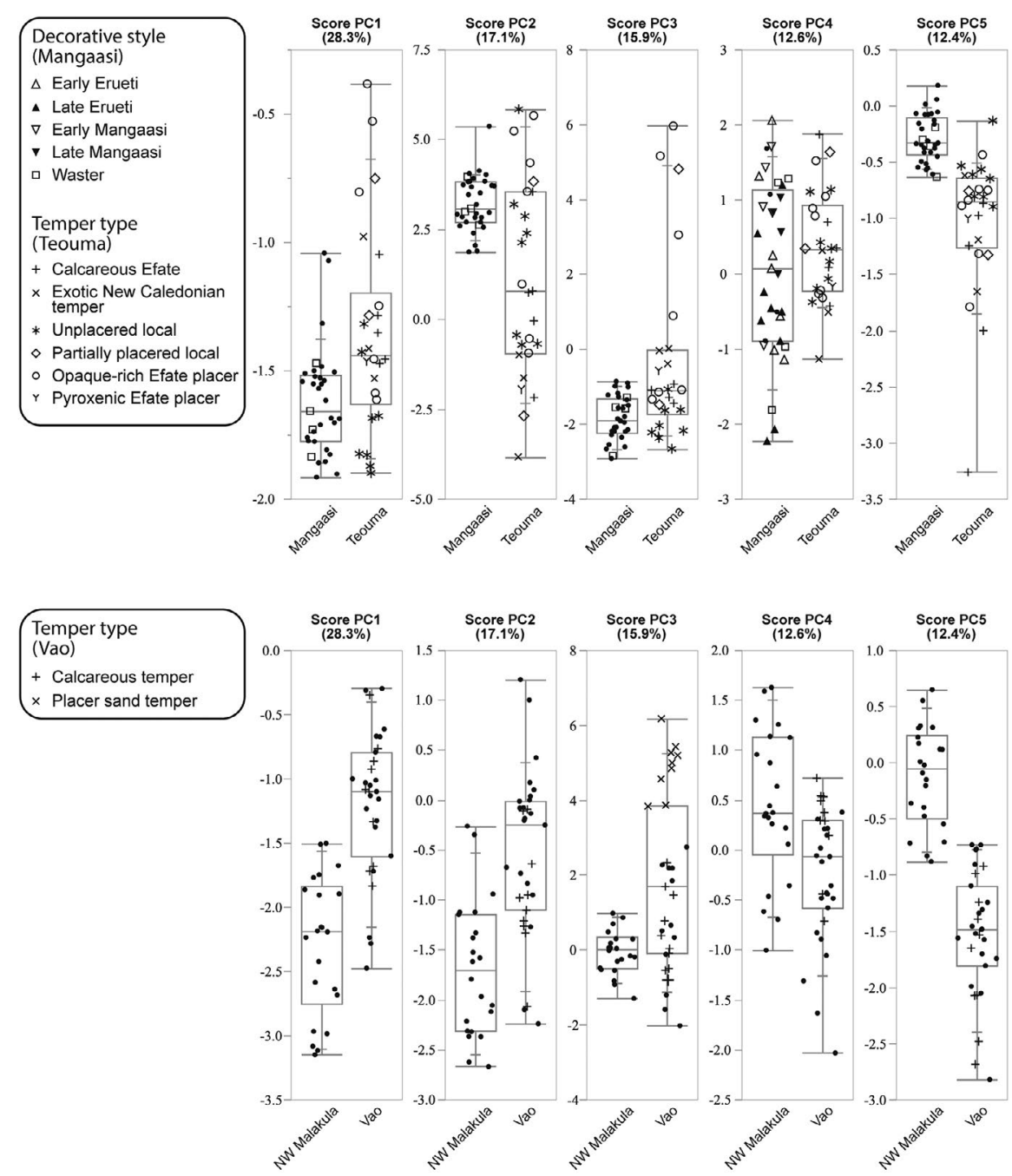

Figure 17.3. Boxplots illustrating the distribution of the principal component scores for the sites on Efate (top) and Malakula (bottom).

The various decorative types encountered at Mangaasi, the temper types identified at Teouma (Dickinson et al. 2013) and two types identified at Vao (calcareous and placer sand) are indicated.

source: Illustration by author.

2 The aim here was to demonstrate the general decrease of variability between decorated Lapita and Post-Lapita pottery assemblages and present insights about the implications of this simplification in terms of behaviour and cultural organisation. The relationship between LA-ICP-MS data and petrography will be addressed in detail in upcoming publications. 
The variability of Mangaasi ceramic samples is, in contrast, highly limited, which is in line with petrographic and macroscopic observations that highlighted the homogeneous character of the collection. Considering that the analysed samples from Mangaasi represent various stages of a robust ceramic sequence spanning about 1700 years, this homogeneity is notable and suggests that the raw material procurement pattern was steadily maintained throughout the occupation. The wasters recovered from Mangaasi also group closely with the tightly distributed group of ceramics (Figure 17.3), which suggests that the vessels not only share compositional similarity but were also all manufactured locally. Regarding the sites located on Malakula, the significant variation in PC2 and PC3 scores of the Vao samples is noticeable from Figure 17.3. In fact, the range of scores for the third component at Vao is the largest of all the sites involved in this project. The distribution of these scores corresponds fairly well with the variants of temper encountered and segregates the non-placer tempers quite efficiently.

As suggested by Figure 17.3 and confirmed by the values for rare earth elements and yttrium (or REY) in Table 17.3, only PC4 scores show comparable dispersion of Lapita and Post-Lapita collections. The comparable ranges of REY values between the homogenous assemblage of Mangaasi, where the same locally available sources of raw material were used continually, and the Teouma ceramics, characterised by great variability in terms of temper type and chemical composition, suggest that the data reflects the natural variability in REY of the raw materials. It also indicates that the variability in REY in clays from a restricted location is as important as the variability encountered in a multitude of raw materials. This echoes the conclusions of recent research documenting the behaviour of rare earth elements in soils that demonstrate that the rare earth element content can vary significantly in relation to pedological layers, the occurrence of specific minerals being the major repository for these elements, and various other factors such as erosion and leaching (Aide and Aide 2012).

Table 17.3. Coefficients of variation (mean-standard deviation) obtained from the various ceramic collections.

\begin{tabular}{|l|c|c|c|c|}
\hline \multicolumn{2}{|c|}{$\begin{array}{c}\text { Mangaasi } \\
\text { (Post-Lapita) }\end{array}$} & $\begin{array}{c}\text { Teouma } \\
\text { (Lapita) }\end{array}$ & $\begin{array}{c}\text { NW Malakula } \\
\text { (Post-Lapita) }\end{array}$ & $\begin{array}{c}\text { Vao } \\
\text { (Lapita) }\end{array}$ \\
\hline Li & 28.9 & 45.2 & 43.8 & 43.8 \\
\hline B & 23.6 & 27.6 & 28.4 & 38.3 \\
\hline Na & 24.8 & 49.8 & 25.5 & 60.7 \\
\hline Mg & 13.8 & 56.2 & 23.8 & 55.4 \\
\hline Al & 6.8 & 15.2 & 5.9 & 16.0 \\
\hline Si & 3.7 & 13.1 & 2.9 & 12.6 \\
\hline K & 16.8 & 37.8 & 51.8 & 54.8 \\
\hline C a & 12.6 & 84.4 & 42.6 & 76.0 \\
\hline Ti & 8.3 & 85.4 & 9.2 & 48.3 \\
\hline V & 25.1 & 83.8 & 23.7 & 60.8 \\
\hline Mn & 53.7 & 97.4 & 29.0 & 57.5 \\
\hline Fe & 13.1 & 59.5 & 12.8 & 45.3 \\
\hline C 0 & 31.6 & 57.9 & 29.2 & 42.8 \\
\hline Ni & 31.4 & 73.2 & 27.9 & 86.9 \\
\hline C u & 17.7 & 30.5 & 26.3 & 19.7 \\
\hline Zn & 16.5 & 88.5 & 16.1 & 50.1 \\
\hline Ga & 9.0 & 25.0 & 10.0 & 20.0 \\
\hline Ge & 24.6 & 55.3 & 29.8 & 60.5 \\
\hline As & 24.2 & 46.0 & 101.2 & 31.1 \\
\hline Rb & 20.2 & 22.9 & 44.3 & 65.0 \\
\hline
\end{tabular}




\begin{tabular}{|l|c|c|c|c|}
\hline \multicolumn{2}{|c|}{$\begin{array}{c}\text { Mangaasi } \\
\text { (Post-Lapita) }\end{array}$} & $\begin{array}{c}\text { Teouma } \\
\text { (Lapita) }\end{array}$ & $\begin{array}{c}\text { NW Malakula } \\
\text { (Post-Lapita) }\end{array}$ & $\begin{array}{c}\text { Vao } \\
\text { (Lapita) }\end{array}$ \\
\hline Sr & 14.1 & 75.2 & 40.7 & 81.4 \\
\hline Y & 58.1 & 27.9 & 39.8 & 31.5 \\
\hline Zr & 17.6 & 56.0 & 10.8 & 18.1 \\
\hline Nb & 18.8 & 53.6 & 184.8 & 78.6 \\
\hline Mo & 30.7 & 60.6 & 13.1 & 32.4 \\
\hline Sn & 11.5 & 43.2 & 70.6 & 34.1 \\
\hline C s & 29.5 & 54.6 & 28.4 & 34.5 \\
\hline Ba & 17.8 & 25.3 & 46.1 & 26.2 \\
\hline La & 43.1 & 52.6 & 35.1 & 27.5 \\
\hline C e & 35.6 & 53.3 & 46.7 & 26.3 \\
\hline Nd & 46.1 & 41.5 & 46.0 & 27.8 \\
\hline Sm & 46.0 & 35.7 & 44.2 & 31.5 \\
\hline Gd & 51.1 & 31.5 & 40.4 & 30.2 \\
\hline Dy & 50.1 & 25.3 & 37.6 & 30.1 \\
\hline Er & 51.6 & 23.8 & 35.0 & 29.4 \\
\hline Yb & 47.7 & 22.6 & 11.8 & 18.7 \\
\hline Hf & 16.2 & 53.9 & 18.6 & 24.2 \\
\hline Pb & 15.2 & 61.1 & 41.7 & 28.4 \\
\hline Th & 18.6 & 79.4 & 174.2 & 86.8 \\
\hline U & 31.1 & 46.1 & & \\
\hline
\end{tabular}

The higher values when comparing Lapita and Post-Lapita collections are highlighted in grey. The Lapita assemblages of Teouma and Vao are generally more variable than Mangaasi and north-west (NW) Malakula, to the exception of $Y$ and the rare earth elements (La, Ce, Nd, Sm, Gd, Dy, Er and Yb).

Source: Author's summary.

\section{Discussion}

Overall, the results reveal that there is a strong tendency for the decorated ceramic collections of the oldest sites to show more variability in terms of chemical composition than more recent sites. This confirms that a greater number of technological styles are represented in Lapita collections, which corresponds to what has been observed previously elsewhere in Oceania in regard to decoration, temper type and vessel forms (e.g. Hunt 1989; Kirch 2000:113; Summerhayes 2000a). The most striking example of this decrease of variability comes from the island of Efate, where the strong variability of the Teouma decorated ceramic assemblage is in strong contrast to the highly homogeneous Mangaasi collection. This is significant considering that the sites represent an almost continuous occupation sequence and that Mangaasi was occupied for a longer period of time. Even when considering that the analysed vessels displaying fine dentatestamped decorations were found in association with burials deposited during the earliest phase of occupation at Teouma, the time gap separating the set of ceramics from Teouma and Mangaasi is at most 140 years. What could explain the loss of this variability in such a relatively short period of time? On Malakula, the gap in time separating the ceramics from Vao (c. 2900 BP (Bedford 2007:189)) and those recovered from the north-west coast (dating back to at most c. 550 BP (Bedford 2006b:67)) is more significant, but the trend for increasing homogeneity in terms of chemical composition through time is also noticeable. 
The nature of the sites must be recognised as a potential contributor to the differences in assemblages between Lapita and immediately Post-Lapita. For example, the Teouma cemetery site has a specialised function while Mangaasi is a more typical settlement site. However, since this gradual homogenisation appears to be a general regional pattern, it is argued that the nature of the site is not the main factor causing this simplification. So what could have motivated this transition from a significant variability of technological styles in decorated Lapita ceramic collections to a more homogeneous ceramic production?

Summerhayes (2000a:234-235) has argued that the technological variability of Lapita assemblages marks the high mobility and the adaptable technology of the potters. In Vanuatu, the great heterogeneity of the results from decorated Lapita collections seems to confirm that Lapita communities were relatively mobile compared to immediately Post-Lapita groups. Not a great deal of movement across the land would, however, be required to produce such heterogeneous compositional profiles given the very important natural variability of the clays (Leclerc 2016). The behaviour of a potter moving about and semi-opportunistically collecting various types of clays as part of other activities (such as searching for food) would be sufficient to result in variable profiles comparable to the results from Lapita collections. This mobility was probably on a relatively small scale, even if rare exotic vessels (those from New Caledonia recovered from Teouma, for example) show that the early Lapita groups that settled in Vanuatu had occasional long-range contacts with other archipelagos. Overall, however, the number of exotic samples at Teouma and Vao is simply not high enough to explain fully the overall variability, even at Teouma where the proportion of imported vessels is relatively high. Compositional analysis shows that the raw materials used in Lapita times were generally less consistent than later, even among samples sharing similar temper types as defined by petrography. The compositional variability observed in Lapita assemblages does not originate from a geographically wider catchment area, but rather from a multiplicity of local/regional technological styles.

Therefore, the idea that the mobility of Lapita communities resulting from their subsistence activities within newly colonised territories had an effect on the variability of raw materials used to produce pottery seems tenable. However, additional explanatory avenues related to social organisation can also be addressed based on the data gathered.

For example, it is conceivable, as has been previously suggested (Ambrose 2007; Summerhayes and Allen 2007), that the compositional variability results from 'systematic' technological experimentation undertaken by potters newly arrived and unfamiliar with the locally available raw materials. The first stage of colonisation would have involved a phase of getting acquainted with the surroundings. In this new landscape, potters possibly had to experiment to determine which raw materials were adequate and better suited for pottery production. The subsequently acquired knowledge would have gradually led to the identification of preferred materials that became increasingly used, resulting ultimately in the homogeneity of the more recent ceramic assemblages. If this scenario provides a plausible explanation, the fact that homogeneous raw materials started to be used approximately at the same time that dentate-stamped decorations and multiple vessel forms stopped being produced suggests that utilitarian considerations related to the potters' familiarisation with local raw materials were not the only factors in play.

The variability observed suggests that Lapita potters did not have any specific prerequisites to guide their raw material selection and that no specific clay was used preferentially. The immediate surroundings of the sites were more commonly exploited, but the overall procurement pattern suggests that opportunistic gathering of clays and sands was adequate. The fact that many different local raw materials have been used to manufacture the majority of decorated Lapita pots indicates that it would have been difficult to control production, considering that many alternative suitable raw materials could be accessed. Consequently, the significant variability of Lapita technological 
styles reveals that there was not any apparent political control or imposed limitations over access to the raw materials used to produce pottery. These conditions also undermine the idea that the compositional variability reflects various specialised potters or workshops. Instead, it indicates quite clearly that the production of Lapita pottery was not specialised, since craft specialisation is usually associated with a certain kind of restriction on the resources and a limited variability of products (Costin and Hagstrum 1995; Rice 1991). This is in line with other studies of Lapita assemblages (Chiu 2003a; Clark 2007; Summerhayes 2000b) and the general argument that the Lapita political economy was open and competitive, which made it difficult to establish conditions suitable for emergent hierarchical relations (Earle and Spriggs 2015).

Overall, the selection of raw material did not seem to be of significant concern for the Lapita potters. As long as they could produce a usable vessel, it did not seem to matter where the clay was from or whether the temper was calcareous, placered, rich in iron oxides or exotic. It is therefore difficult to conceive that the technological aspects of the dentate-stamped pottery contributed to the prestigious character of the vessels. This suggests that prestige was more likely to have been associated with the skills required to manufacture the vessels rather than from the vessels themselves. This recalls Chiu's similar views in regard to the decorative Lapita face motifs: 'The image of a motif may have differed, but the message it contained remained the same' (Chiu 2007:259). In our case, the raw materials differed but useful dentate-stamped pottery kept being produced.

The manufacture of dentate-stamped ceramics must have also represented an important social investment considering the variety of dentate-stamped motifs, their intricacy and the large size of some of the vessels (Ambrose 2007; Clark 2007). Consequently, the underlying social organisation must have been adequately adapted to support this type of production. Whether only women potters were involved in the process (Marshall 1985) or every member of the community participated, the episodes of pottery production must have represented a stress on the community and appropriate social measures must have been in place to ensure that basic subsistence tasks were still undertaken in parallel. The important shift observed between Lapita and Post-Lapita indicates that the social structure was also probably modified conjointly with the settlement pattern. Such social transformation undoubtedly contributed to the observed increase in technological efficiency and the cessation of lengthy dentate-stamped decoration. In these new conditions, perhaps the people who used to be associated with pottery production had additional time-consuming tasks — related to horticulture and pig-rearing for example-that prevented them investing as much time as before on pottery production.

Lastly, the sharp contrast between the homogeneity of immediately Post-Lapita pottery and the heterogeneity of decorated Lapita vessels should definitely not be seen as 'laziness' or growing disinterest in the technological aspects of pottery production. In fact, it likely shows the opposite. The rupture is so abrupt that it must illustrate a strategy to differentiate subsequent cultural production from the former Lapita political, economic and ideological structures. The knowledge about using various clays and temper types was not instantly forgotten. Yet, the production quickly became homogenised. Such sudden change is suggestive of the deliberate rejection of both the message and its linked medium (Siorat 1990). The immediately Post-Lapita homogeneity in terms of pottery technological styles suggests not only that the Lapita system did not mean anything to these communities anymore but also that they wanted to distance themselves from it. 


\section{Technological simplification as part of a general process}

According to van der Leeuw et al. (1991), dominant technological styles are maintained if the 'unquestioned assumptions' related to the ways pottery should be manufactured and used by the society in which the potters operate remain the same. In consequence, the fact that pottery technological styles were transformed supports the argument that substantial sociocultural transformation occurred around the transition between Lapita and Post-Lapita. Furthermore, the fact that traditional ways of manufacturing pottery were modified at the same time as other important aspects of society strengthens the argument: the increasing regionalisation of pottery decorations (Bedford and Clark 2001); the changes in distribution patterns for Banks Island obsidian (Reepmeyer 2008; Reepmeyer et al. 2011); the divergence in languages between the north and the south of Vanuatu (Tryon 1996); and modifications in dietary intake and interindividual diversity in burial rites (Bedford et al. 2011; Valentin et al. 2016; Valentin et al. 2014) all point toward significant changes between Lapita and immediately Post-Lapita periods.

Considering that pottery manufacture, funerary rites and dietary habits are generally conservative practices (Arnold 1992:159; Valentin et al. 2014), it is suggested that these changes most probably affected every aspect of the society: economic, symbolic, religious and political. Whether these changes resulted from adaptive responses to changing climatic conditions or the arrival of new populations as suggested by Valentin et al. (2016) is an interesting question. Generally, the fact that a similar process of simplification of decorated Lapita pottery assemblages has been recorded from the Arawes (Summerhayes 2000a), the Mussau group (Hunt 1989; Kirch 2000:113) and now in Vanuatu attests that there was some kind of continuing communication between the communities and that a general regionalisation process was occurring. Over time, populations increased and the inhabitants of particular islands and island groups settled in by modifying the environment and by becoming more adept at procuring sufficient resources and finding suitable marriage partners locally. The eventual focus on local interactions and the development of local social communities ultimately led to the breakdown of the original Lapita entity. Eventually, the desire to invest significant amounts of time and energy in an obsolete system defining their old identity was not so enticing anymore as these settlements thrived and became self-sustainable. 


\begin{tabular}{|c|c|c|c|c|c|c|c|c|c|c|c|c|c|c|c|c|c|c|c|c|c|c|c|c|c|c|}
\hline & & & & & & & & & & & & & & & & & & & & & & & & & \\
\hline & & 工 & 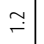 & 낟 & & & & & 过 & & $\simeq$ & & $8=$ & $\stackrel{m}{=}$ & & 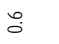 & & & & & & & $\stackrel{\sim}{2}:$ & $=7$ & & \\
\hline a & & 望 & 䇇 & & & 9 & & $\approx$ & 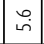 & $\stackrel{\alpha}{m}$ & $\underset{\forall}{*}$ & & in & 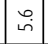 & $\mid \begin{array}{l}0 \\
0\end{array}$ & 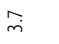 & & & & & & & $\therefore$ & $\stackrel{\alpha}{2}$ & & \\
\hline t5 & & $\approx$ & & & $\bar{i}$ & & & 28 & $\bar{N}$ & $\stackrel{\infty}{\sim}$ & $\begin{array}{lll}n_{2}\end{array}$ & & i & $\stackrel{i}{*}$ & \begin{tabular}{|l|l|}
$\infty$ & \\
\hdashline
\end{tabular} & & & & & & & & $\bar{m}$ & 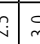 & & \\
\hline & & & & & & & & \pm & \begin{tabular}{|l|l|} 
I \\
\end{tabular} & & & & $\bar{\pi}$ & & & & & & & & & & 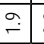 & & & \\
\hline & & $\overline{\mathrm{i}}$ & & 87 & $=$ & $\begin{array}{l}\overrightarrow{0} \\
0.1\end{array}$ & 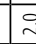 & \pm & \begin{tabular}{|l|}
\multirow{2}{*}{} \\
\end{tabular} & $\cong$ & $\begin{array}{l}25 \\
25\end{array}$ & & 2 & i & \begin{tabular}{|l|} 
\\
\end{tabular} & & & & & & & $\stackrel{1}{\simeq}$ & 9 & 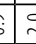 & & \\
\hline & & mi & & $\Rightarrow 5$ & 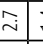 & \pm & $\sqrt{n}$ & $\stackrel{m}{2}$ & F & $\stackrel{\infty}{\stackrel{\infty}{\sim}}$ & $\begin{array}{lll}3 & 3 \\
\end{array}$ & & $\left.\right|_{m} ^{+}$ & $\overline{\mathrm{n}}$ & \begin{tabular}{|l|l|}
2 \\
\end{tabular} & 2 & & & & & \pm & $\begin{array}{ll} \\
\stackrel{2}{2}\end{array}$ & $\begin{array}{ll}\vec{m} \\
\end{array}$ & 2 & & 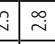 \\
\hline & & $\bar{m}$ & $\begin{array}{l} \\
\end{array}$ & & $\stackrel{2}{\sim}:$ & \pm 3 & ${ }_{m}^{\circ}$ & $\overline{\bar{N}}$ & $\bar{q}$ & 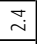 & 5 & & $\bar{m}$ & 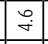 & $\underline{m}$ & 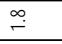 & & & & & & $\stackrel{2}{+}$ & iे & & & \\
\hline 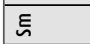 & & $m$ & & & $\bar{\sim}$ & 27 & $\sqrt{m}$ & 2 & $\bar{m}$ & $\dot{I}$ & 7 & & $m$ & जी & \begin{tabular}{|l|l|} 
\\
\end{tabular} & 9 & & & & & & $\bar{i}$ & $\vec{i}$ & & & \\
\hline$z$ & & $\stackrel{\vec{\beth}}{\beth}$ & 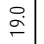 & 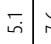 & $\stackrel{\circ}{\sim}$ & go & $\exists$ & $\infty$ & $\stackrel{\stackrel{\sim}{\beth}}{\cong}$ & $\infty_{\infty}^{+}$ & $\underline{2}$ & & 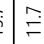 & $\stackrel{I}{\Xi}$ & $\mid$\begin{tabular}{|}
$\infty$ \\
$\stackrel{\sim}{\sim}$
\end{tabular} & 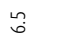 & & & & & $\bar{\sigma}$ & 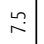 & $\stackrel{.}{\circ}$ & o & ${ }_{\infty}^{\infty}$ & 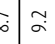 \\
\hline ๘ & & 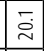 & 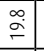 & $\begin{array}{ll}0 \\
\circ\end{array}$ & $\stackrel{0}{ \pm}$ & $\therefore:$ & 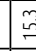 & 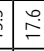 & \begin{tabular}{|l|}
0 \\
0
\end{tabular} & $\bar{\Xi}$ & $\underset{\Xi}{ \pm}$ & & $\stackrel{\infty}{\simeq}$ & $\stackrel{i}{i}$ & \begin{tabular}{|l|}
$\infty$ \\
$\infty$ \\
\end{tabular} & 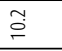 & & & & & $\stackrel{\circ}{\circ}$ & & $\overline{ \pm}$ & & & 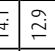 \\
\hline $\mathbb{E}$ & f & $\bar{\infty}$ & 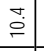 & 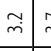 & $\vec{m}$ & $\bar{m}$ & 구 & 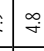 & कु & f & 27 & & $\stackrel{2}{\sim}$ & $\Xi$ & \begin{tabular}{|l|} 
\\
\end{tabular} & $q$ & & & & & F & 它 & 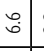 & & & \\
\hline ๓ & $\mid \begin{array}{l}\infty \\
\substack{\sigma \\
\sigma}\end{array}$ & 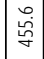 & 紫 & & 䓌 & $\begin{array}{l}\infty \\
\vdots \\
\vdots \\
\vdots\end{array}$ & ${ }_{7}^{\infty}$ & 瓷 & $\mid \begin{array}{l}0 \\
0 \\
\tilde{n}\end{array}$ & 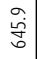 & $\stackrel{\vec{\exists}}{\vec{g}}$ & & 总 & 紊 & $\tilde{\Xi}$ & $\stackrel{\mathbb{E}}{\Xi}$ & & & & m & ธิ่ & $\begin{array}{l}\bar{\alpha} \\
\stackrel{\alpha}{\infty}\end{array}$ & 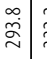 & & & 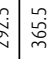 \\
\hline 气 & 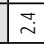 & \begin{tabular}{|l|l} 
\\
0 \\
0
\end{tabular} & of & $8:$ & तi & 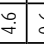 & b & $\stackrel{m}{2}$ & $\cong$ & \begin{tabular}{|l|l|}
$\infty$ \\
$\stackrel{-}{-1}$
\end{tabular} & 5 & & $\bar{F}$ & a & $\bar{\pi}$ & $a$ & & & & & & g & \begin{tabular}{|l|l|} 
\\
$\square$
\end{tabular} & 7 & & \\
\hline Бక & 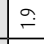 & 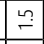 & $\begin{array}{l} \\
\end{array}$ & $m$ & \pm & $=7$ & 7 & \pm & $\stackrel{4}{\square}$ & \pm & $\approx$ & & 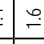 & i & $\cong$ & 5 & & & & & & $i$ & $\equiv$ & 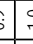 & & $=7$ \\
\hline$\stackrel{\varrho}{\Sigma}$ & 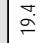 & ga & $\begin{array}{l}\infty \\
\stackrel{\infty}{\infty} \mid\end{array}$ & $\stackrel{-}{-1}$ & 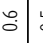 & 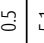 & : & $=$ & $\hat{o}$ & $\stackrel{\circ}{-}$ & $\stackrel{\Xi}{\square}$ & 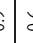 & 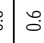 & p. & $\stackrel{\circ}{-}$ & $c$ & & & & & $\stackrel{?}{\longrightarrow}$ & $\simeq$ & $\stackrel{-}{-}$ & 3 & & $=4$ \\
\hline$\hat{z}$ & 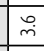 & $\bar{m}$ & $m$ & $\begin{array}{lll} & 7 \\
\end{array}$ & $\therefore$ & $\overline{\bar{i}}$ & I & 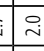 & $\stackrel{i}{\circ}$ & $\stackrel{\sim}{\sim}$ & $\begin{array}{ll} \\
\end{array}$ & & 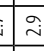 & $\hat{m}$ & \begin{tabular}{|l|l|}
\multicolumn{2}{|c|}{} \\
\end{tabular} & 5 & & & & & & \begin{tabular}{|l|}
$\infty$ \\
$\dot{\sim}$ \\
\end{tabular} & $\vec{m}$ & 2 & & 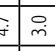 \\
\hline$\approx$ & 㐫 & $\begin{array}{l}\circ \\
\end{array}$ & '. & $\frac{m}{5}$ & है: & aे & D & 吾 & $\begin{array}{l}f \\
\end{array}$ & $m$ & 吕 & & గi & $\bar{\infty}$ & \begin{tabular}{|l|} 
\\
\end{tabular} & a & 놎 & & & & & $\bar{I}$ & $\stackrel{0}{\circ}$ & & & $\stackrel{a}{a}$ \\
\hline 7 & $\stackrel{\infty}{\sim}$ & 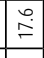 & ָ̊. & $?$ & $\stackrel{\text { İ }}{\stackrel{2}{2}}$ & 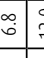 & 它 & $\stackrel{\circ}{\circ}$ & \begin{tabular}{|l|} 
\\
\multirow{\sim}{*}{} \\
\end{tabular} & $\begin{array}{l} \pm \\
\dot{I} \\
\end{array}$ & 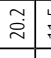 & & 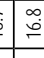 & $\underset{\sim}{\stackrel{\sim}{\sim}}$ & \begin{tabular}{|l|}
5 \\
\end{tabular} & 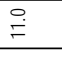 & $\underline{0}$ & 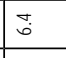 & 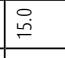 & $f$ & o. & 2 & 声 & $\exists$ & & 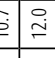 \\
\hline$=$ & 它 & 亲 & ఖ్ల & 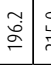 & 总 & 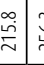 & $\frac{D}{7}$ & 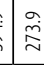 & 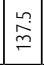 & E & 望 & & : & 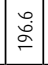 & 守 & $\widetilde{\approx}$ & $\stackrel{\infty}{\infty}$ & & & & $\frac{2}{2}$ & & : & & & $\begin{array}{l}0 \\
n \\
n\end{array}$ \\
\hline ๙ิ & $\vec{m}$ & $\overrightarrow{\dot{\sigma}}$ & 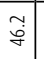 & \begin{tabular}{l|l} 
\\
\end{tabular} & $f$ & $\begin{array}{l}\infty \\
\bar{\sigma}\end{array}$ & $\underset{\approx}{\approx}$ & $\mathscr{2}$ & \begin{tabular}{l|} 
\\
\\
\end{tabular} & 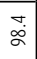 & $\overrightarrow{\dot{m}}$ & & $\stackrel{\vec{m}}{\vec{m}}$ & 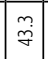 & $\mid \begin{array}{l}\mid \\
\vdots \\
0\end{array}$ & $\lesssim$ & & & & & 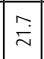 & $\stackrel{\circ}{\stackrel{\circ}{\rightleftarrows}}$ & $\stackrel{\sim}{\sim}$ & & & $\stackrel{n}{n}$ \\
\hline$z$ & 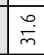 & 吕 & 㐘 & $\stackrel{\natural}{7}$ & 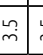 & ली: & 9 & a & \begin{tabular}{|l|l} 
\\
\end{tabular} & $\bar{\sigma}$ & 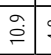 & & $m_{m}^{m}$ & $\begin{array}{l} \\
\end{array}$ & \begin{tabular}{|l|}
$\infty$ \\
\\
\end{tabular} & 5 & & & & & $\overbrace{\infty}^{\infty}$ & $\begin{array}{ll} \\
\end{array}$ & 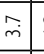 & $y$ & & 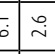 \\
\hline 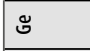 & $\stackrel{\infty}{\sim}$ & $\approx$ & \begin{tabular}{l|l|l|} 
\\
$\infty$
\end{tabular} & 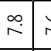 & 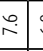 & $\begin{array}{lll}\infty & 3 \\
\infty & 3\end{array}$ & 5 & 7 & $\bar{F}$ & $\stackrel{\infty}{m}$ & +2 & & 9 & \begin{tabular}{|l|l|}
+ \\
\end{tabular} & \begin{tabular}{|l|} 
\\
\\
\end{tabular} & 9 & & & & & $\stackrel{+}{m}$ & 它 & $\stackrel{\sim}{ \pm}$ & & & 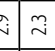 \\
\hline 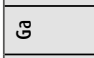 & $\approx$ & \begin{tabular}{|l|l}
$\infty$ \\
$\mathbb{d}$ \\
\end{tabular} & 方 & $\tilde{z}$ & $\tilde{\sim})$ & $\sqrt{3}$ & $\stackrel{\infty}{\stackrel{\infty}{\sim}}$ & 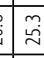 & $\begin{array}{l}\vec{i} \\
\end{array}$ & $\mathcal{I}$ & $\stackrel{a}{\dot{\sim}}$ & & 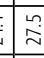 & 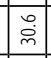 & 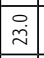 & 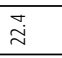 & $\stackrel{m}{m}$ & $\approx$ & & $\stackrel{i}{i}$ & 号 & $\overrightarrow{\mathrm{m}}$ & $\begin{array}{ll}\overrightarrow{2} \\
\stackrel{2}{2}\end{array}$ & & & $\bar{i}$ \\
\hline$\approx$ & 莞 & \begin{tabular}{|l|l}
\multirow{2}{*}{} \\
\end{tabular} & 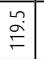 & $\begin{array}{l}5 \\
\dot{0}\end{array}$ & 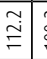 & 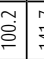 & 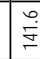 & 豆 & 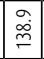 & 离 & $\overrightarrow{\dot{I}}$ & & 雍 & 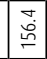 & 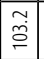 & o & $\stackrel{\square}{a}$ & $\stackrel{\bar{\Xi}}{\underline{\Xi}}$ & a & \begin{tabular}{|l}
$\mid \widetilde{a}$ \\
$\simeq$
\end{tabular} & 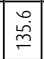 & 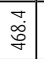 & $\bar{q}$ & & & $\stackrel{a}{0}$ \\
\hline उ & $\stackrel{\infty}{\rightleftharpoons}$ & $\stackrel{\substack{\infty \\
=}}{\stackrel{2}{*}}$ & ò & $\stackrel{\infty}{\underset{\sim}{\beth}}$ & : & 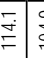 & 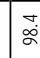 & 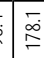 & $\equiv$ & $\begin{array}{ll}\infty \\
\infty \\
\infty\end{array}$ & 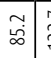 & & 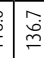 & 总 & 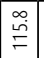 & 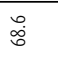 & ت্ & $\stackrel{m}{\Delta}^{m}$ & 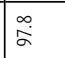 & 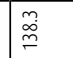 & 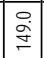 & 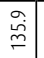 & : & $\stackrel{\sim}{\approx}$ & & $\frac{2}{0}$ \\
\hline $\bar{z}$ & 色 & $\stackrel{\neg}{\stackrel{\sim}{\sim}}$ & $m^{m}$ & $\stackrel{i}{i}$ & \begin{tabular}{l|} 
\\
$\triangle$ \\
$\triangle$
\end{tabular} & $\stackrel{\circ}{\circ}$ & 胥 & $\stackrel{m}{\sim}$ & $\lesssim$ & $\stackrel{\sim}{\sim}$ & 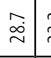 & & $\stackrel{a}{i}$ & $\begin{array}{l}\dot{m} \\
\dot{m}\end{array}$ & \begin{tabular}{|c|c}
$\infty$ \\
0 \\
$m$
\end{tabular} & $\bar{\infty}_{\text {je }}$ & 0 & $\mid$ & வீ: & log & 总 & 宁 & 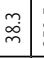 & & & 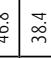 \\
\hline 8 & 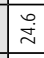 & \begin{tabular}{|l|}
$m$ \\
$m$ \\
$m$
\end{tabular} & $\begin{array}{l} \\
\stackrel{0}{*}\end{array}$ & จิ & 常 & $\stackrel{n}{a}$ & $\infty$ & 品 & 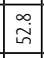 & 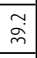 & fos & & 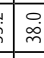 & 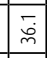 & \begin{tabular}{|l|}
$\tilde{g}$ \\
\end{tabular} & $\stackrel{m}{i}$ & $\simeq$ & 过 & 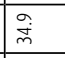 & ||$^{f}$ & 름 & $\overline{\vec{y}}$ & ঐ̊. & $\lambda^{\infty}$ & & 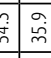 \\
\hline 这 & 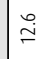 & $\stackrel{\rho}{\stackrel{\rho}{\rho}}$ & $\stackrel{\leftrightarrow}{=}$ & $\stackrel{g}{=}$ & $\hat{=}$ & = & $\check{\check{I}}$ & $\exists$ & 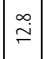 & $\stackrel{\circ}{=}$ & $\stackrel{\square}{=}$ & & $\stackrel{\circ}{\stackrel{\rho}{9}}$ & $\stackrel{\sim}{\simeq}$ & $\exists$ & $\tilde{\infty}$ & a & í & 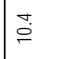 & $\approx$ & 官 & $\stackrel{\vec{m}}{\vec{m}}$ & $\tilde{\cong}$ & 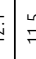 & & $y$ \\
\hline 을 & $\overline{0}$ & $\overline{0}$ & & 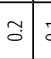 & -1 & 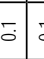 & 5 & $\tilde{a}$ & 5 & - & $\dot{0}$ & & s. & 5 & $\tilde{s}$ & $\tilde{\sigma}$ & & & & & 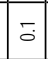 & $\tilde{s}$ & $\overline{0}$ & & & 3 \\
\hline$>$ & $\mid$ & 离 & 苟 & $\frac{\hat{6}}{m}$ & 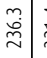 & 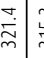 & J & 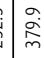 & 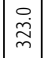 & 岀 & $\begin{array}{l}0 \\
\stackrel{0}{\sim} \\
\sim\end{array}$ & & 过 & $\mid \begin{array}{l}\overrightarrow{2} \\
\overrightarrow{2}\end{array}$ & $\frac{m}{m}$ & 旁 & & 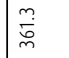 & & 总 & $\left|\begin{array}{c}0 \\
\dot{y}\end{array}\right|$ & 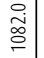 & $\begin{array}{l}\stackrel{0}{\infty} \\
\stackrel{m}{m}\end{array}$ & & & $\frac{0}{10}$ \\
\hline : & $\cong$ & $\stackrel{\circ}{=}$ & $=$ & $\simeq 7$ & $=$ & $\bar{F}=$ & 19 & $=$ & $m$ & $F$ & $\begin{array}{c}\text { o. } \\
\end{array}$ & & 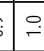 & $=$ & $=$ & o & $\stackrel{s}{-}$ & 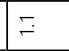 & $=$ & $\stackrel{\leftrightarrow}{-}$ & $\stackrel{.}{\circ}$ & 望 & 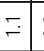 & 7 & & $\stackrel{\infty}{r}$ \\
\hline 8 & $\bar{\sim}$ & $\stackrel{\sim}{\sim}$ & 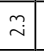 & $\hat{0}$ & \pm & $\stackrel{:}{=}$ & $\stackrel{m}{m}$ & 28 & a & $\stackrel{m}{\square}$ & $\stackrel{\vec{m}}{\mathrm{~m}}=$ & & $=$ & $\stackrel{0}{\circ}$ & \begin{tabular}{|l|} 
\\
\end{tabular} & $\stackrel{m}{i}$ & & $\div$ & & $\stackrel{4}{4}$ & 7 & 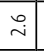 & $\begin{array}{ll}n \\
n\end{array}$ & 3 & & $\begin{array}{ll}7 \\
\end{array}$ \\
\hline 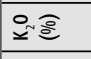 & $\stackrel{\infty}{\circ}$ & $\stackrel{m}{=}$ & 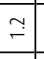 & $\stackrel{\vec{m}}{\mathrm{~m}}:$ & $=$ & $\overline{\bar{i}}:$ & 9 & $\stackrel{2}{2}$ & $\stackrel{2}{\sim}$ & 尔 & $\because$ & & a & a & \begin{tabular}{|l|l|} 
\\
\end{tabular} & $\stackrel{\square}{\square}$ & & ì & & $\stackrel{2}{-}$ & \begin{tabular}{|l|l|} 
\\
- \\
\end{tabular} & $\begin{array}{ll}2 \\
5 \\
0\end{array}$ & $\stackrel{m}{-2}$ & & & $=\overline{7}$ \\
\hline ํㅡㄴ & L & 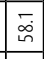 & & 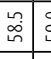 & 品: & 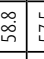 & 6 & $\sqrt{n}$ & \begin{tabular}{|l|}
$\dot{i}$ \\
in
\end{tabular} & 鬲 & \begin{tabular}{|c|c|}
$\infty$ & \\
$\infty$ & \\
\end{tabular} & & ( & 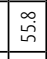 & 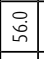 & $\bar{\sigma}$ & & & & & 悹 & $\begin{array}{l} \\
\end{array}$ & 它: & & & 5 \\
\hline$\frac{0}{4} \bar{\varrho}$ & $\mathbb{z}$ & $\stackrel{\sim}{\sim}$ & $\stackrel{\sim}{\sim}$ & i் & 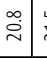 & 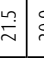 & $b_{1}^{\circ}$ & $\stackrel{2}{g}$ & $\begin{array}{l}\infty \\
\stackrel{\infty}{\sim} \\
\end{array}$ & $\bar{\sigma}$ & $a$ & & $\stackrel{\circ}{\bar{\lambda}}$ & 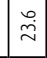 & $\hat{\sigma}$ & $\stackrel{\circ}{\circ}$ & $=$ & 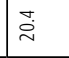 & & + & $\Xi$ & 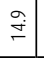 & $\begin{array}{l}\infty \\
\stackrel{\infty}{-} \\
-\end{array}$ & & & $\stackrel{2}{2}$ \\
\hline 高高 & 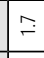 & $\stackrel{i}{i}$ & $\stackrel{\circ}{\circ}$ & $\stackrel{2}{2}$ & $\approx$ & $=$ & $\stackrel{n}{\sim}$ & $m$ & 放 & i & \begin{tabular}{|c|c|}
\multirow{\sim}{*}{} \\
\end{tabular} & & 4 & N & $\bar{N}$ & $\stackrel{\text { i }}{\text { ind }}$ & $=$ & $\bar{i}$ & & & $m$ & $\approx$ & $\begin{array}{ll}4 \\
\end{array}$ & 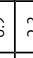 & & 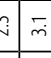 \\
\hline$\frac{0}{2}$ & $\stackrel{\rho}{\sigma}$ & $\stackrel{2}{\tau}$ & & $\stackrel{\sim}{\sim}$ & $\stackrel{2}{\sim}$ & $\stackrel{2}{q}$ & $\infty$ & $\infty$ & & $\bar{i}$ & \begin{tabular}{|l|}
$\div$ \\
\end{tabular} & & $=$ & $\stackrel{0}{-1}$ & $\approx$ & $\bar{\pi}$ & $\stackrel{\text { m }}{\text { in }}$ & ঐे & $\Rightarrow$ & $\stackrel{\infty}{-}$ & of & . & \pm & & & a \\
\hline$\infty$ & 要 & ฮี & $\checkmark$ & \begin{tabular}{l|l}
0 \\
0
\end{tabular} & $\stackrel{\circ}{\circ}$ & 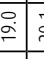 & 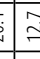 & 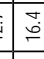 & \begin{tabular}{|l|} 
\\
\end{tabular} & $\stackrel{\sim}{\sim}$ & \begin{tabular}{|l|}
$\circ$ \\
$\propto$ \\
\end{tabular} & & 政 & $\overline{\dot{\rho}}$ & $\begin{array}{l}\infty \\
\dot{\sim} \\
\end{array}$ & 悹 & 5 & $\hat{\sigma}$ & & & $\begin{array}{l}\infty \\
\mathbb{z} \\
\end{array}$ & 金 & 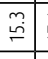 & f & & $=$ \\
\hline$=$ & $\sim$ & I & $\begin{array}{l} \\
\end{array}$ & 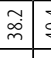 & $\begin{array}{l}\vec{a}+5 \\
\dot{\sigma}\end{array}$ & $\bar{\Sigma}=$ & 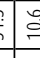 & $\underline{m}$ & \begin{tabular}{|l|l|} 
\\
$\infty$ \\
$\infty$
\end{tabular} & $\bar{I}$ & \begin{tabular}{|l|} 
\\
$\dot{\sim}$
\end{tabular} & & $\bar{d}$ & $\frac{\partial}{m}$ & \begin{tabular}{|l|l|}
\multicolumn{2}{|c|}{} \\
\end{tabular} & $\infty_{\infty}^{\infty}$ & & & & & \begin{tabular}{|l|} 
म \\
\end{tabular} & (5) & $\Xi$ & $\exists$ & & 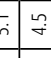 \\
\hline " & & & & & & & & & & & & & & & & & & & & & 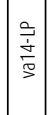 & & & & & \\
\hline & & & & & & & & & & & & & & & & & & 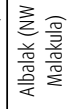 & & 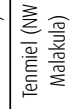 & & & & & & \\
\hline
\end{tabular}

\section{terra australis 52}




\begin{tabular}{|c|c|c|c|c|c|c|c|c|c|c|c|c|c|c|c|c|c|c|c|c|c|c|c|c|c|c|c|c|c|c|}
\hline & & & & & & & & & & & & & & & & & & & & & & & & & & & & & & \\
\hline & & & & & & & & & & & & & & & 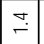 & & & & $\tau$ & & & & & $\approx$ & & 0 & & 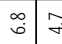 & & \\
\hline & & & & & & & & & $\stackrel{\infty}{\rightarrow}$ & & & & & & 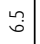 & if & & & $=7$ & & \begin{tabular}{|l|l|l}
4 \\
$\infty$
\end{tabular} & $\vec{\sim}$ & & 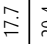 & & & $=$ & & & \\
\hline & & & & & & & & & & & & & & & $\overrightarrow{\mathrm{i}}$ & & $\underset{\sim}{\infty}$ & & 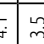 & & g & \begin{tabular}{|l|} 
品 \\
\end{tabular} & 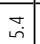 & $v^{q}$ & & & \begin{tabular}{|l|l}
$\infty$ \\
|
\end{tabular} & \begin{tabular}{l|l} 
\\
\llcorner
\end{tabular} & & \\
\hline & & & & & & & & & & & & & & & & & & & & & & & & & & & & & & \\
\hline & & & & & $F$ & & & & & & & & & $\stackrel{i}{i}$ & $\tilde{\mathrm{I}}$ & i & & & & & $\cong$ & T. & & & & & & $\stackrel{\circ}{\longrightarrow}$ & & \\
\hline & & : & & $\underset{\sim}{\infty}$ & $\bar{i}$ & 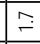 & $\tilde{m}$ & $\bar{i}$ & $\overline{\mathrm{i}}$ & & 5 & & & $\tilde{m}$ & $\begin{array}{l}\infty \\
\stackrel{\infty}{m}\end{array}$ & $\bar{m} \mid$ & वे? & 77 & $=\pi$ & J & $\stackrel{i}{i}$ & $F$ & \begin{tabular}{|l|l|} 
\\
\end{tabular} & \pm & & 5 & $F$ & \begin{tabular}{|l|}
$\stackrel{\infty}{\sim}$ \\
\end{tabular} & & \\
\hline & & & & & $\approx$ & & & $\stackrel{i}{i}$ & 9 & & & & & \begin{tabular}{|l|l} 
\\
$m$ \\
\end{tabular} & 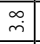 & $\overrightarrow{\mathrm{i}}$ & & & $=\pi$ & 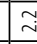 & $\stackrel{i}{i}$ & - & $\omega^{\circ}$ & 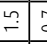 & & & & & & \\
\hline & & & & & 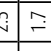 & & & $\stackrel{i}{i}$ & 官 & & & & & $\stackrel{\mathrm{m}}{\mathrm{m}}$ & 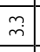 & 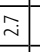 & & $\pi$ & & & $\stackrel{i}{\sim}$ & \begin{tabular}{|l} 
\\
\end{tabular} & & & & & & $\stackrel{2}{n}$ & & \\
\hline & & $\stackrel{\circ}{\because}$ & & $\infty$ & in & \% & $\stackrel{\circ}{\circ}$ & $m$ & $\cong$ & & 赵 & $\infty$ & & 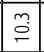 & 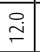 & $\approx$ & $\approx$ & $: 7$ & $f \stackrel{4}{9}$ & $\underset{F}{\stackrel{F}{F}}$ & 里 & 亲 & 灾 & $\begin{array}{l}\infty \\
\infty\end{array}$ & 7 & $\approx 2$ & 2 & $\begin{array}{lll}m & 7 \\
& \end{array}$ & & \\
\hline & & 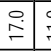 & & & $=\frac{\infty}{2}$ & $\stackrel{m}{\stackrel{m}{=}}$ & & $\stackrel{m}{=}$ & $\stackrel{0}{\circ}$ & & $\frac{2}{2}=$ & $\cong$ & & $\vec{I}$ & \begin{tabular}{|l|l} 
\\
\\
\end{tabular} & $\exists$ & & & 0 & & $\stackrel{\stackrel{\rho}{m}}{\circ}$ & \begin{tabular}{|l|} 
\\
\end{tabular} & ن. & : & & $\hat{m}$ & \begin{tabular}{|l|l} 
\\
$\stackrel{\sigma}{q}$
\end{tabular} & \begin{tabular}{l|l}
$m$ \\
$\dot{q}$
\end{tabular} & & \\
\hline & & & & 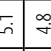 & : & F & 吕 & gे & 它 & & & น & & \begin{tabular}{|l|}
5 \\
5
\end{tabular} & \begin{tabular}{|l|} 
\\
\end{tabular} & 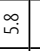 & 它 & \begin{tabular}{l|l}
$\cos ^{\circ}$ & \multicolumn{1}{|c|}{} \\
\end{tabular} & $\therefore$ & $\bar{E}$ & 周 & 2 & $\stackrel{m}{ \pm}$ & $\begin{array}{ll}\infty \\
\infty\end{array}$ & 5 & 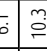 & \begin{tabular}{|l|l|} 
\\
\end{tabular} & $\stackrel{7}{\Xi} \underset{m}{\Xi}$ & & \\
\hline$\approx$ & 릴 & $\begin{array}{l}0 \\
\dot{m}\end{array}$ & & & 安 & $\underset{\sim}{\stackrel{N}{N}}$ & & 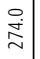 & 苂 & & 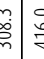 & $\bar{c}$ & & $\left|\begin{array}{c}\bar{\infty} \\
\bar{m}\end{array}\right|$ & & $\stackrel{ \pm}{\approx}$ & & & $\left(\begin{array}{l}\infty \\
0\end{array}\right.$ & & 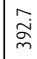 & 总 & 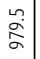 & ఏ్ర & & & & $\begin{array}{l}\frac{f}{2} \\
\frac{1}{2} \\
\vdots \\
0\end{array}$ & & \\
\hline & & $\stackrel{8}{\circ}$ & & & $=\stackrel{\infty}{\sim}$ & 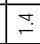 & के & $\begin{array}{ll}{ }^{\prime} \\
\end{array}$ & 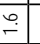 & & $a$ & & & $\begin{array}{ll} \\
0\end{array}$ & 媖 & | & : & \begin{tabular}{l|l}
$\infty$ \\
$\infty$
\end{tabular} & $=7$ & $\stackrel{2}{\square}$ & $\stackrel{5}{\mp}$ & $\infty$ & i) & $=9$ & 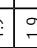 & $=9$ & 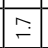 & जी & & \\
\hline & & 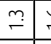 & & & \pm & 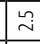 & \pm & $\stackrel{9}{g}$ & 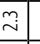 & & $=0$ & & & $\cong$ & $\bar{F}$ & $\begin{array}{l} \\
\end{array}$ & $=1$ & $=5$ & $=7$ & 9 & $\stackrel{\infty}{\stackrel{\infty}{\sim}}$ & I & $\vec{m}$ & 97 & 5 & $\bar{i}$ & $\bar{\lambda}$ & $\bar{m}$ & & \\
\hline & & 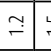 & $\stackrel{2}{\square}$ & 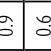 & J & $F$ & \begin{tabular}{|l|}
2 \\
\end{tabular} & $\stackrel{m}{=}$ & - & & 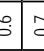 & $\underset{-}{+}$ & & \begin{tabular}{|l|l|} 
\\
\end{tabular} & $\hat{0}$ & \begin{tabular}{l|l} 
\\
\end{tabular} & $\stackrel{2}{9}$ & \begin{tabular}{l|l}
$\infty$ \\
0
\end{tabular} & $=2$ & ga & $\mp$ & $\approx$ & $=$ & 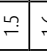 & 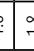 & 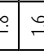 & $\stackrel{m}{2}$ & 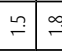 & & \\
\hline & & बे: & $\bar{g}$ & & $m$ & 字 & $\stackrel{P}{+}$ & $\vec{m}$ & 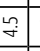 & & 15 & & & \begin{tabular}{|l|}
\multirow{2}{*}{} \\
\end{tabular} & $\vec{i}$ & $\stackrel{\sim}{\sim}$ & m: & mas & $m$ & & \begin{tabular}{|l} 
\\
\end{tabular} & \begin{tabular}{|l} 
\\
\end{tabular} & की & 字: & & $f_{j}^{\infty}$ & & 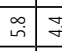 & & \\
\hline & & $i$ & & $\frac{2 n}{2}$ & $=2 \frac{2}{2}$ & बें & \begin{tabular}{|l}
$u$ \\
$\tilde{o}$ \\
0
\end{tabular} & $\begin{array}{l}a \\
\dot{a}\end{array}$ & 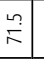 & & $=$ & \pm & & \begin{tabular}{|l|} 
\\
\\
\end{tabular} & 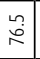 & 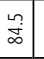 & 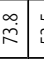 & $\tilde{n}$ & 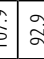 & 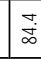 & In & 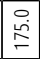 & 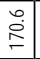 & 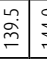 & 5 & & $\bar{E}$ & 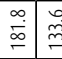 & & \\
\hline & & & & 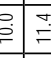 & 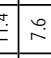 & a & 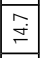 & $\begin{array}{l}\infty \\
\infty\end{array}$ & \begin{tabular}{|c|}
$\infty$ \\
$\infty$
\end{tabular} & & 5 & $\left.\right|_{\infty} ^{\infty}$ & & $\bar{\square}$ & \begin{tabular}{|l|l|} 
\\
\end{tabular} & $\underset{z}{ \pm}$ & \pm & & $\stackrel{I}{I}$ & & 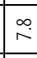 & \begin{tabular}{|l|l}
\multirow{+}{*}{} \\
\end{tabular} & $\stackrel{m}{d}$ & $\overline{5}$ & & Si & F & $\widehat{5}$ & & \\
\hline$=$ & $\dot{\gamma}^{\prime}$ & $\begin{array}{l}\stackrel{\tilde{\Xi}}{\Xi} \\
\Xi\end{array}$ & & & $\begin{array}{c}0 \\
0 \\
0\end{array}$ & 总 & & 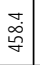 & 妾 & & : & 8 & & $\mid \begin{array}{l}0 \\
0 \\
i=n \\
i n n\end{array}$ & 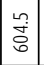 & 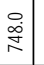 & & $\frac{1}{2}$ & $\stackrel{8}{\circ}$ & & \begin{tabular}{|l} 
离 \\
a
\end{tabular} & $\mid \begin{array}{l}0 \\
0 \\
0 \\
\end{array}$ & & 言要 & & & & & & \\
\hline & & 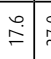 & 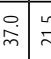 & & $\begin{array}{l}\dot{v} \\
\dot{v}\end{array}$ & $\ddot{\sim}$ & \begin{tabular}{l}
0 \\
\hdashline \\
\hdashline \\
\end{tabular} & $\stackrel{+}{\dot{\sim}}$ & 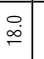 & & 5 & $\stackrel{\infty}{=}$ & & 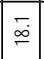 & $\vec{i}$ & 定 & ֻٕن & $\begin{array}{l} \pm \\
\pm\end{array}$ & $\dot{0} \bar{m}$ & & 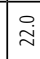 & 水 & $\begin{array}{l}0 \\
:\end{array}$ & $\stackrel{\infty}{\sim} \underset{\sim}{\sim}$ & & & & $\begin{array}{l}8 \\
8\end{array}$ & & \\
\hline & & & & g & In & $\stackrel{\circ}{\circ}$ & 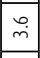 & 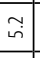 & 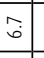 & & 15 & & & \begin{tabular}{|l|}
\multirow{8}{*}{} \\
\end{tabular} & \begin{tabular}{|l|} 
\\
$\infty$ \\
\end{tabular} & $\overline{0}$ & in: & \begin{tabular}{l|l} 
\\
\end{tabular} & \begin{tabular}{l|l}
$\infty$ \\
0 \\
0
\end{tabular} & m & $\bar{\infty}$ & $\begin{array}{l}\circ \\
\end{array}$ & 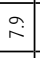 & : & & $\begin{array}{l}0 \\
0\end{array}$ & \begin{tabular}{|r}
5 \\
\end{tabular} & 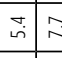 & & \\
\hline & & & & & $\bar{n} \bar{m}$ & $\begin{array}{ll}\infty \\
\infty \\
\end{array}$ & & 8 & $\bar{\nabla}$ & & 67 & $\stackrel{ \pm}{\sim}$ & & $\bar{m}$ & \begin{tabular}{|l|}
$\infty$ \\
$\stackrel{2}{-}$ \\
\end{tabular} & 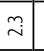 & $\stackrel{\sim}{m}$ & {$\left[\begin{array}{c|c}a \\
2\end{array}\right.$} & $\begin{array}{ll}0_{0} \\
\end{array}$ & & a & $\bar{N}$ & $\stackrel{+}{\circ}$ & $\stackrel{5}{m}$ & & $\pi$ & 足 & 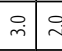 & i & \\
\hline & & 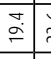 & & $\overline{\mathrm{Z}}$ & $\stackrel{2}{8}$ & 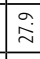 & $\stackrel{+}{\stackrel{2}{\sim}}$ & 足 & 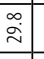 & & +5 & 2. & & \begin{tabular}{|l|l}
\multirow{2}{*}{} \\
\end{tabular} & 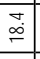 & 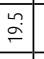 & 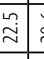 & $\stackrel{8}{0}$ & 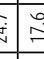 & 9 & 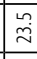 & \begin{tabular}{|c|}
5 \\
5
\end{tabular} & $\begin{array}{l}\vec{\lambda} \\
\end{array}$ & $\stackrel{2}{\grave{2}}$ & 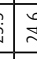 & & \begin{tabular}{|l|l}
\multirow{2}{*}{} \\
\end{tabular} & \begin{tabular}{|l|l} 
\\
$\vdots$
\end{tabular} & 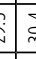 & \\
\hline 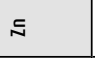 & & & 熍 & $\stackrel{0}{0}$ & 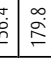 & స్ల్ల & \begin{tabular}{|l}
2 \\
$\dot{m}$ \\
\end{tabular} & 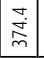 & $\begin{array}{l}\overrightarrow{\mathrm{v}} \\
\vec{y}\end{array}$ & & $\frac{1}{2}$ & 吕 & 过 & \begin{tabular}{|l|}
\multirow{2}{0}{} \\
\end{tabular} & 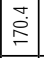 & 怘 & 品 & 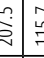 & $\hat{\varepsilon}=\begin{array}{l}2 \\
a\end{array}$ & $\mid \begin{array}{l}a \\
\beth\end{array}$ & $\mid \stackrel{\circ}{\stackrel{i}{i}}$ & $\begin{array}{l}\infty \\
\dot{\alpha} \\
\end{array}$ & 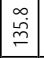 & 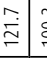 & $\begin{array}{l}\infty \\
\vdots \\
0\end{array}$ & & 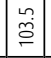 & 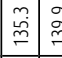 & 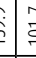 & \\
\hline & & $\stackrel{\ddot{z}}{\rightleftarrows}$ & & $\frac{1}{2}$ & $\underline{1}$ & 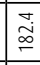 & : & 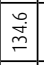 & 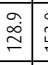 & & 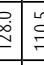 & $\alpha$ & $\frac{2}{2}$ & \begin{tabular}{|l|l} 
\\
\\
0
\end{tabular} & \begin{tabular}{|l}
0 \\
\end{tabular} & 吉 & 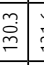 & 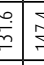 & $\bar{\infty}$ & 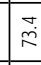 & 宝 & 产 & 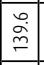 & 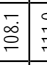 & $\underline{\xi}$ & $\stackrel{7}{E}$ & $\approx$ & \begin{tabular}{c|c}
0 \\
0 \\
\hdashline
\end{tabular} & 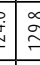 & \\
\hline $\bar{z}$ & & $\frac{F}{F}$ & & & $\dot{h}$ & $\bar{y}$ & 吕 & 总 & 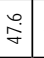 & $\stackrel{\Im}{\mathcal{J}}$ & 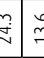 & ga & & \begin{tabular}{|l|l|} 
\\
f \\
\end{tabular} & \begin{tabular}{|l|r}
$\vec{f}$ \\
\end{tabular} & 离 & $\stackrel{8}{\vec{F}}$ & 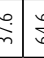 & $\begin{array}{l}\underbrace{\circ}_{0} \\
0\end{array}$ & & $\overline{\text { s. }}$ & $\stackrel{g}{\check{E}}$ & $\bar{\Xi}$ & $\stackrel{\square}{\Xi}$ & ఓ. & 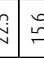 & $\bar{\sigma}$ & 2 & & \\
\hline 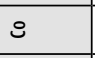 & & $\frac{m}{\sim}$ & 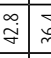 & & 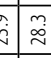 & Fे & $\ddot{z}$ & \begin{tabular}{|l|} 
\\
\\
\\
\end{tabular} & $\vec{\Delta}$ & & $\pi$ & $\Delta$ & 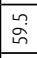 & \begin{tabular}{|l|l|}
0 \\
$d$
\end{tabular} & $\vec{i}$ & $\stackrel{m}{d}$ & $\vec{m}$ & 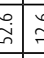 & $\stackrel{8}{i}$ & \begin{tabular}{|l}
0 \\
\end{tabular} & 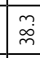 & \begin{tabular}{|l|l|}
\multicolumn{1}{|c|}{} \\
\end{tabular} & 吕 & 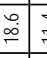 & 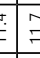 & 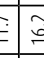 & $\begin{array}{ll}m \\
m\end{array}$ & $\stackrel{2}{2}$ & 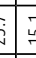 & \\
\hline on: & 年 & $\stackrel{f}{ \pm}$ & 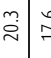 & 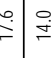 & 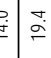 & $\ddot{\sim}$ & 占 & $\stackrel{\tilde{m}}{m}$ & 品 & $\stackrel{\alpha}{7}$ & $\begin{array}{l}\infty_{0} \\
\end{array}$ & $m_{m}^{\circ}$ & & 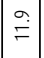 & $\overline{\dot{m}}$ & $\stackrel{\Xi}{=}$ & 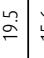 & 6 & \begin{tabular}{l}
$: \infty$ \\
\hdashline
\end{tabular} & & 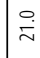 & $\infty$ & $\stackrel{+}{\circ}$ & $\bar{s}$ & & & $\approx$ & $\stackrel{\infty}{\infty}$ & 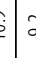 & \\
\hline & & & & & $\overline{5}$ & $\approx$ & 3 & $\begin{array}{l}m \\
0\end{array}$ & $\begin{array}{l}n \\
0\end{array}$ & & 5 & $\approx$ & & 5 & 5 & $\overline{0}$ & & 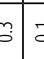 & $5:$ & & $\approx$ & - & $\tilde{o}$ & $\overline{0}$ & & & $\overline{0}$ & 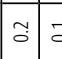 & & \\
\hline & ำ & 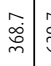 & 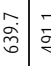 & & & $\frac{\bar{m}}{\bar{\infty}}$ & 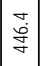 & 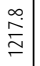 & 容 & & 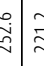 & 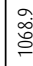 & 量 & $\mid \begin{array}{l}2 \\
0 \\
0 \\
n\end{array}$ & $\left|\begin{array}{c}n \\
0 \\
m \\
m\end{array}\right|$ & 鼠 & 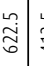 & 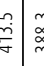 & $:$\begin{tabular}{l}
7 \\
\hdashline \\
\hdashline
\end{tabular} & & 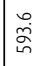 & $\frac{m}{0}$ & $\mid \begin{array}{c}0 \\
\stackrel{0}{0} \\
-0\end{array}$ & 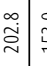 & 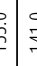 & e & 兽 & $\begin{array}{l}\frac{\pi}{\circ} \\
=\end{array}$ & & \\
\hline 0 & $\underset{\sim}{*}$ & 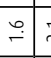 & $\overline{\mathrm{v}}$ & $\simeq$ & $\geq \stackrel{2}{=}$ & $\approx$ & $\approx$ & $\stackrel{\vec{m}}{\dot{m}}$ & $\stackrel{n}{m}$ & 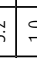 & $=$ & i & $\stackrel{\infty}{m}$ & $\simeq$ & $\stackrel{m}{=}$ & $\stackrel{1}{\circ}$ & 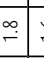 & $6=$ & $=$ & & $\stackrel{\circ}{\sim}$ & \begin{tabular}{|l}
$\infty$ \\
\end{tabular} & $\stackrel{-1}{-1}$ & $\stackrel{-}{\circ}$ & & 5 & $\begin{array}{l}\infty \\
0 \\
\end{array}$ & $\stackrel{-}{-}$ & $\because$ & \\
\hline & & $\stackrel{\mathscr{\Omega}}{\simeq}$ & $\widetilde{\sigma}$ & & $\begin{array}{lll}n \\
\end{array}$ & $\hat{m}$ & \begin{tabular}{|l|}
\multirow{2}{*}{} \\
\end{tabular} & F & $\bar{\nabla}$ & & 65 & r & $t$ & 5 & 莎 & $\stackrel{\infty}{\sim}$ & inj & $6=$ & 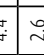 & $\stackrel{\infty}{\sim}$ & $\begin{array}{l}\infty \\
\sigma\end{array}$ & $\approx$ & के & : & & 42 & a & 2 & 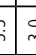 & \\
\hline$=5$ & & $\stackrel{\infty}{\infty}$ & $\stackrel{m}{\sim}$ & $F$ & $=\stackrel{\circ}{-}$ & $\hat{o}$ & $\begin{array}{l}m \\
\end{array}$ & : & $\stackrel{0}{\circ}$ & 5 & $=0$ & 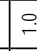 & t? & 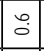 & \begin{tabular}{|l|l} 
\\
\end{tabular} & \pm & $\underset{m}{2}:$ & $=$ & $=$ & $\stackrel{\infty}{-}$ & $\begin{array}{l}\infty \\
0 \\
0\end{array}$ & $\because$ & $\cong$ & $:=$ & 7 & $=0$ & $\stackrel{m}{\sim}$ & $\because 9$ & 7 & \\
\hline $\bar{n}$ & & \begin{tabular}{l|l}
$\vec{a}$ \\
$\dot{a}$
\end{tabular} & \begin{tabular}{|l|l|}
\multirow{g}{*}{$g$} \\
$\dot{q}$
\end{tabular} & & 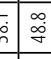 & \begin{tabular}{|l}
\multirow{8}{*}{} \\
\end{tabular} & 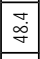 & 买 & \begin{tabular}{|c|c|}
\multirow{2}{*}{} \\
$\stackrel{9}{*}$
\end{tabular} & & 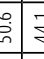 & & & \begin{tabular}{|l|}
$\overrightarrow{5}$ \\
\end{tabular} & 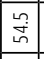 & 窇 & $\begin{array}{l}9 \\
\dot{\sigma}\end{array}$ & th: & $\frac{5}{6}$ & \begin{tabular}{|ll}
$\circ$ \\
\end{tabular} & 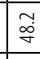 & \begin{tabular}{|l|} 
\\
$\tilde{b}$
\end{tabular} & \begin{tabular}{|l|} 
\\
\\
\\
\end{tabular} & 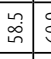 & 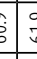 & $\frac{5}{6}$ & \begin{tabular}{|l|}
5 \\
5
\end{tabular} & \begin{tabular}{|l|l|l} 
& 0 \\
$b$ & 0
\end{tabular} & & \\
\hline$\frac{\pi}{\alpha}$ & $\stackrel{m}{\mp}$ & 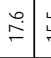 & $\stackrel{n}{2}$ & & $\begin{array}{l}0 \\
\dot{0}\end{array}$ & $\stackrel{\text { I }}{\sim}$ & 吕 & $\grave{\Xi}$ & 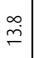 & 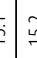 & $f$ & & & 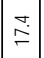 & $\Xi$ & $\stackrel{\square}{\circ}$ & 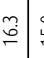 & fil & $\hat{s}$ & & 臽 & $\sim$ & $\tilde{\sim}$ & $\overline{\dot{I}}$ & & & $\approx$ & \begin{tabular}{|l|l}
\multirow{2}{*}{} & \\
\end{tabular} & $\tau$ & \\
\hline 这 & 5 & & 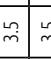 & & & $\stackrel{\sim}{\mathrm{i}}$ & $\begin{array}{l} \\
\end{array}$ & $\overline{\mathrm{i}}$ & $\bar{\sim}$ & & 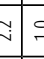 & - & & \begin{tabular}{|l|l|} 
\\
\end{tabular} & \begin{tabular}{|l|l|}
\multirow{2}{*}{} \\
\end{tabular} & $\therefore$ & $\overrightarrow{\mathrm{m}}$ & m. & $y=$ & $F$ & $\stackrel{\infty}{\stackrel{\infty}{-}}$ & 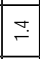 & $\stackrel{m}{-}$ & $=$ & & $=$ & $F$ & $\because=$ & 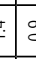 & \\
\hline en & t. & & & & & í & \begin{tabular}{|l|l|} 
\\
\end{tabular} & 资 & 望 & 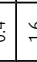 & $=$ & & & $\therefore$ & 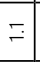 & & 8 & & $\because \approx$ & & : & $\cong$ & $=$ & 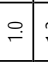 & & & & $\therefore$ & & \\
\hline$\infty$ & 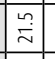 & 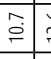 & $\begin{array}{l}\dot{\underline{u}} \\
\end{array}$ & & 它 & $\stackrel{m}{\stackrel{m}{i}}$ & 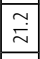 & \pm & $\stackrel{8}{ \pm}$ & & 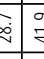 & $\underset{f}{f}$ & & $\stackrel{\text { }}{\circ}$ & כึ) & 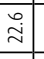 & 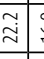 & . & $=\begin{array}{l}\infty \\
\vdots \\
\vdots\end{array}$ & $\stackrel{m}{I}$ & 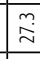 & 苛 & 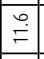 & $\overrightarrow{\mathrm{z}}$ & & & \begin{tabular}{|c|c|}
$\infty$ \\
$\infty$ \\
\hdashline
\end{tabular} & $\left(\begin{array}{l}\stackrel{i}{i}) \\
2\end{array}\right.$ & 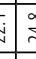 & \\
\hline & f & $\begin{array}{lll} & 5 \\
\end{array}$ & 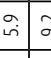 & & $\dot{5}$ & $\underset{\forall}{+}$ & $\begin{array}{l} \\
\end{array}$ & m & $\begin{array}{l}\text { ले } \\
\end{array}$ & & \begin{tabular}{l|l}
$\infty$ & $\bar{n}$ \\
\end{tabular} & & & $\begin{array}{ll}0 \\
\end{array}$ & \begin{tabular}{|l|l|}
\multirow{2}{*}{} \\
\end{tabular} & $\begin{array}{l} \\
\stackrel{\sim}{*}\end{array}$ & $\begin{array}{lll}0 & 1 \\
0 & 1\end{array}$ & & $\bar{a}$ & & 每 & वे & \begin{tabular}{|l|} 
\\
\end{tabular} & $\bar{\square}$ & & & & & & \\
\hline 类 & & & & & & & & & & & & & & & & & & & & & & & & & & & & & & \\
\hline & & & & & & & & & & & & & & & & & & & & & & & & & & & & & & \\
\hline
\end{tabular}




\begin{tabular}{|c|c|c|c|c|c|c|c|c|c|c|c|c|c|c|c|c|c|c|c|c|c|c|c|}
\hline$p$ & 。. & $\underset{z}{\simeq}$ & & & & & & & & & & & & & & & $\Xi$ & ò & $\stackrel{+}{\dot{+}}$ & $\begin{array}{l}\infty \\
0 \\
0\end{array}$ & $\stackrel{8}{i}$ & $\stackrel{\sim}{i}$ & $\begin{array}{l}\hat{a} \\
\end{array}$ \\
\hline$E$ & oิ & $\begin{array}{l}\infty \\
\infty \\
\infty \\
0\end{array}$ & $F$ & 5 & & :. & 泣 & 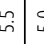 & 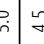 & $\stackrel{1}{\sim}$ & 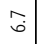 & $\vec{F}:$ & $f$ & ${ }^{\infty}$ & $\bar{\alpha}$ & $\mid \begin{array}{l}\infty \\
0 \\
0\end{array}$ & g & 约 & $\hat{\omega}$ & $\stackrel{n}{n}$ & $\stackrel{n ?}{ }$ & $\underset{F}{F}$ & $\stackrel{\infty}{\infty}$ \\
\hline a & 审 & $\stackrel{\sim}{\stackrel{\sim}{2}}$ & 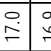 & 6 & $\stackrel{\infty}{\infty}$ & 恣 & $\stackrel{\infty}{\infty}$ & \begin{tabular}{l}
0 \\
\hdashline
\end{tabular} & $=$ & i & $\bar{\Xi}$ & $\stackrel{\infty}{\infty}$ & I & $\underset{d}{d}$ & $\bar{a}$ & 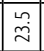 & $\stackrel{n}{\alpha}$ & $\stackrel{\infty}{\underset{\sim}{\sim}}$ & $\overline{\mathbf{i}}$ & $\stackrel{n}{\text { ì }}$ & $\approx$ & गे & $\stackrel{q}{q}$ \\
\hline tz & तn & 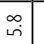 & $\begin{array}{c}\infty \\
m^{\infty}=\end{array}$ & 8 & 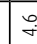 & \begin{tabular}{|l|l|}
$\infty$ \\
$\infty$
\end{tabular} & 9 & $\bar{n}$ & $g$ & 5 & $\begin{array}{l}\infty \\
\substack{\infty \\
n}\end{array}$ & 73 & \begin{tabular}{l|l} 
\\
0
\end{tabular} & in & פ & 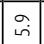 & 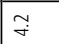 & $\frac{\sigma}{g}$ & 8 & 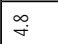 & mi & $\bar{\gamma}$ & 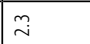 \\
\hline \pm & $\bar{J}$ & ò & \begin{tabular}{l|l}
$m$ & 7
\end{tabular} & $=\sigma^{\infty}$ & & $\stackrel{i}{i}$ & $\bar{i}$ & $\frac{m}{2}=$ & $\infty_{\infty}^{\infty}$ & & הi & $\infty$ & 7 & $m$ & $\stackrel{\infty}{-\infty}$ & $\bar{i}$ & $\tilde{\lambda}$ & $\bar{F}$ & $\stackrel{\circ}{\circ}$ & $\bar{\nu}$ & $\stackrel{n ?}{\square}$ & $\bar{\Sigma}$ & $\stackrel{i}{i}$ \\
\hline 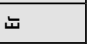 & $\stackrel{i}{i}$ & $\begin{array}{l}\infty \\
\infty \\
\infty\end{array}$ & $\because 9$ & 5 & $\stackrel{\circ}{\square}$ & \begin{tabular}{|c|c|}
2 \\
2
\end{tabular} & $\stackrel{i}{i}$ & $\begin{array}{ccc}0 \\
-5\end{array}$ & $3 a$ & $\digamma$ & $\overline{\mathrm{i}}$ & $=$ & 4 & $\cong$ & $\stackrel{\square}{\circ}$ & $\frac{2}{2}$ & $\bar{i}$ & 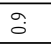 & 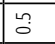 & $\overline{\bar{i}}$ & $\stackrel{\mid J}{I}$ & $\stackrel{i}{i}$ & $\dot{\sigma}$ \\
\hline $\bar{a}$ & m & $\stackrel{m}{m}$ & 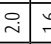 & 67 & $\stackrel{\infty}{-\infty}$ & $\bar{y}$ & $\frac{p}{y}$ & $=7$ & 87 & i & $\begin{array}{l}\mathrm{m}_{\mathrm{m}} \\
\end{array}$ & $\overrightarrow{\mathrm{i}}$ & 27 & I & $\underset{\sim}{\infty}$ & $\tilde{m}$ & $\stackrel{m}{m}$ & $\stackrel{J}{\perp}$ & $\stackrel{8}{\circ}$ & $\begin{array}{l} \\
\dot{m}\end{array}$ & $\overline{\mathbf{I}}$ & 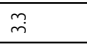 & $\bar{m}$ \\
\hline 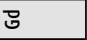 & $\stackrel{+}{+}$ & $\stackrel{m}{m}$ & $\bar{\pi}$ & $6=$ & & $\frac{8}{8}$ & 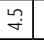 & $=7$ & $=7$ & $\stackrel{\overrightarrow{\mathrm{N}}}{\mathrm{N}}$ & 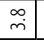 & $\bar{m}:$ & $\begin{array}{c}\dot{0} \\
\end{array}$ & 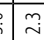 & 2 & $\tilde{m}$ & $\ddot{m}$ & 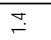 & gे & $\stackrel{\infty}{\infty}$ & $\stackrel{i}{i}$ & $\vec{m}$ & $\stackrel{\infty}{\sim}$ \\
\hline $\bar{\Xi}$ & of & $\mid$ & 4 & 25 & & $\begin{array}{ll}m \\
\omega_{n}\end{array}$ & $\begin{array}{ll}3 \\
n \\
n\end{array}$ & 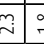 & $=$ & $\bar{m}$ & fo & $\left.\begin{array}{c}\infty \\
\infty\end{array}\right]$ & $\begin{array}{c}8 \\
\end{array}$ & 글 & io & $\bar{f}$ & \begin{tabular}{|l|l}
0 \\
\end{tabular} & 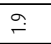 & J & $\bar{f}$ & $\stackrel{i}{i}$ & $\vec{q}$ & 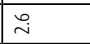 \\
\hline $\bar{z}$ & $\stackrel{\infty}{\infty}$ & 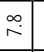 & $\begin{array}{lll} \\
\dot{E}\end{array}$ & 8 & $\stackrel{402}{=}$ & 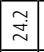 & $\stackrel{n}{\approx}$ & \pm 0 & 9 & \begin{tabular}{|l}
$\stackrel{\circ}{\circ}$ \\
$\stackrel{2}{*}$
\end{tabular} & 品 & $\stackrel{5}{\stackrel{5}{*}}$ & $=\infty$ & $\stackrel{\infty}{ \pm}$ & $\stackrel{\infty}{\infty}$ & \begin{tabular}{|l|}
$\infty$ \\
$\stackrel{\infty}{\infty}$ \\
\end{tabular} & $\hat{i}$ & $\tilde{\infty}$ & ì & $\stackrel{n}{\stackrel{n}{2}}$ & 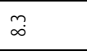 & 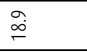 & $\begin{array}{l}\infty \\
\infty \\
\end{array}$ \\
\hline ๘ & $\begin{array}{l}a \\
\dot{b} \\
\dot{d}\end{array}$ & ¿े. & $\bar{\Xi}$ & $\stackrel{\square}{\circ}$ & m & \begin{tabular}{|c|}
$n$ \\
nn
\end{tabular} & $\overline{\sigma o}$ & & $\mathrm{~s}$ & 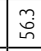 & 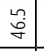 & $\frac{7}{\tilde{q}}$ & 4 & $\begin{array}{l}\infty \\
m\end{array}$ & م. & \begin{tabular}{|l|}
$a$ \\
$\dot{q}$ \\
\end{tabular} & \begin{tabular}{|l} 
\\
\\
n.
\end{tabular} & $\stackrel{0}{\underset{d}{d}}$ & 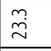 & 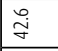 & $\stackrel{亏}{E}$ & 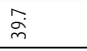 & 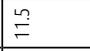 \\
\hline$\Xi$ & $\stackrel{m}{\varrho}$ & $\stackrel{1}{\circ}$ & 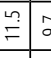 & 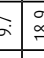 & $\stackrel{m}{g}$ & 至 & $\overline{\bar{i}}$ & 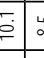 & 65 & 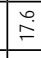 & $\overrightarrow{\dot{\nu}}$ & $\stackrel{5}{2}$ & $\stackrel{n}{2}$ & \pm & $\stackrel{m}{a}$ & \begin{tabular}{|l|l} 
\\
$\stackrel{\infty}{\circ}$ \\
\end{tabular} & $\stackrel{\rho}{\infty}$ & 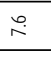 & ఫ़ & 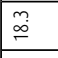 & $\stackrel{2}{i}$ & $\begin{array}{l}\stackrel{\circ}{\circ} \\
\stackrel{\circ}{\circ}\end{array}$ & g \\
\hline ఐ & 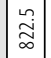 & $\begin{array}{c}\frac{\alpha}{\infty} \\
\frac{\infty}{\infty}\end{array}$ & 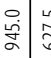 & : & 年 & 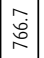 & 怘 & & 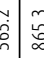 & స్త్ & \begin{tabular}{l}
$\frac{2}{2}$ \\
\hdashline \\
\hdashline
\end{tabular} & 资 & 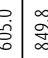 & 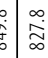 & $\begin{array}{l}\infty \\
\stackrel{\infty}{a}\end{array}$ & 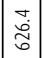 & $\stackrel{\equiv}{\equiv}$ & 过 & 岕 & ณૂี & 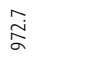 & 客 & 莺 \\
\hline$\approx$ & \begin{tabular}{|c|} 
I \\
\end{tabular} & 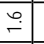 & $\begin{array}{lll}9 & 7\end{array}$ & $=0$ & 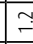 & $\stackrel{-1}{-}$ & $\stackrel{9}{\circ}$ & 77 & 87 & $\stackrel{i}{i}$ & $\stackrel{m}{m}$ & $=7$ & $7 a$ & 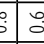 & $\stackrel{9}{\circ}$ & \begin{tabular}{|l} 
\\
\end{tabular} & $\stackrel{\square}{\square}$ & $\vec{i}$ & 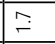 & $\begin{array}{l}\infty \\
\stackrel{\infty}{+}\end{array}$ & $\bar{i}$ & 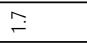 & $\bar{\sigma}$ \\
\hline 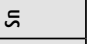 & 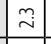 & $\stackrel{\sim}{\sim}$ & î] & $1 \%$ & & I & $\bar{i}$ & $\overline{\mathrm{i}}$ & ע. & \begin{tabular}{|l|} 
\\
\end{tabular} & $\stackrel{\sim}{\sim}$ & $=9$ & 20 & $\begin{array}{l}0.0 \\
\end{array}$ & $\stackrel{m}{m}$ & 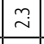 & 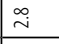 & $\tilde{i}$ & $\underset{i}{i}$ & $\tilde{\Sigma}$ & $\simeq$ & $\bar{i}$ & a \\
\hline$\stackrel{̊}{\Sigma}$ & $\stackrel{ \pm}{\mp}$ & $\stackrel{\sim}{\sim}$ & $\stackrel{\Rightarrow}{=}$ & $=7$ & 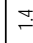 & $\stackrel{\infty}{-}$ & \pm & $=7$ & $=7$ & $\stackrel{\sim}{i}$ & $\stackrel{i}{\sim}$ & $\stackrel{\circ}{\square}:$ & $=7$ & $=7$ & $\bar{\sim}$ & $\underset{\sim}{.}$ & $\bar{i}$ & $\stackrel{\circ}{\leftarrow}$ & $\stackrel{\Xi}{\check{L}}$ & $\approx$ & $\stackrel{\infty}{\infty}$ & I & aे \\
\hline $\bar{z}$ & 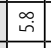 & \begin{tabular}{|c|}
\multirow{2}{*}{} \\
\end{tabular} & \begin{tabular}{l|l}
$\vec{m}$ & 7 \\
\end{tabular} & 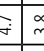 & & \begin{tabular}{|l|}
$\infty$ \\
$\infty$ \\
\end{tabular} & $\begin{array}{l} \\
\end{array}$ & 89 & 87 & ] & $\bar{\sigma}$ & $\frac{7}{7}$ & $\begin{array}{l}0 \\
0\end{array}$ & \begin{tabular}{|l|l} 
\\
\end{tabular} & \begin{tabular}{|l|l} 
\\
$\infty$
\end{tabular} & $\overline{6}$ & m & $\dot{i n}$ & ì & $\bar{f}$ & $\stackrel{\mathrm{i}}{\mathrm{N}}$ & F & \begin{tabular}{|l|l|} 
\\
\end{tabular} \\
\hline$\approx$ & 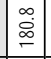 & 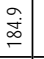 & $\begin{array}{ll}n \\
=\end{array}$ & $\stackrel{0}{\circ}$ & J & $\begin{array}{l}2 \\
\vdots \\
\vdots\end{array}$ & \begin{tabular}{l}
0 \\
0 \\
\hdashline
\end{tabular} & 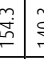 & 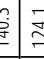 & \begin{tabular}{l} 
岁 \\
\hdashline \\
\hdashline
\end{tabular} & 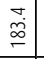 & 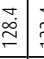 & 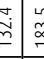 & 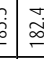 & $\frac{\bar{g}}{d}$ & 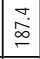 & \begin{tabular}{|l}
$\infty$ \\
$\stackrel{m}{m}$ \\
\end{tabular} & 总 & 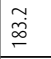 & 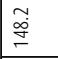 & 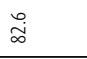 & ஹ̊ & 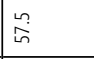 \\
\hline$>$ & \begin{tabular}{|l|l|} 
\\
\end{tabular} & กี & \begin{tabular}{c|c}
$\infty$ & 5 \\
\hdashline & \\
\end{tabular} & 5 & a & ind & 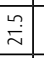 & 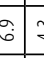 & 9 & 芦 & \begin{tabular}{|l|}
\multirow{2}{*}{} \\
\end{tabular} & 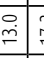 & 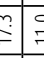 & $\begin{array}{ll}2 \\
2\end{array}$ & $\equiv$ & \begin{tabular}{|l|}
$f$ \\
\end{tabular} & \begin{tabular}{|l|}
$\underline{0}$ \\
\end{tabular} & in & $\begin{aligned} \\
\end{aligned}$ & $\underset{I}{ \pm}$ & $\stackrel{\infty}{\Sigma}$ & 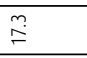 & $\underset{\sim}{\infty}$ \\
\hline $\bar{n}$ & $\begin{array}{l}a \\
\substack{a \\
0}\end{array}$ & స్ & $\begin{array}{lll}\widetilde{Z} \\
0\end{array}$ & 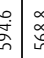 & 我 & $\stackrel{m}{\underline{m}}$ & 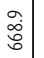 & 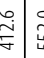 & $v_{n}^{2}$ & 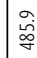 & 瓷 & కేర్ & 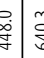 & I & $\stackrel{\infty}{i}$ & 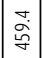 & $\mid \begin{array}{l}n \\
\text { 管 }\end{array}$ & 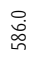 & 啇 & 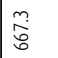 & : & ঞু & 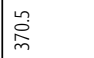 \\
\hline$\stackrel{\imath}{\approx}$ & $\stackrel{a}{\grave{b}}$ & fี & $\stackrel{m}{\dot{q}} \cong$ & $\dot{\sigma}$ & $\int_{m}^{2}$ & \begin{tabular}{|l|}
$\infty$ \\
0 \\
\end{tabular} & 足 & 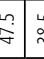 & 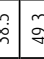 & $\approx$ & $\stackrel{\vec{\gamma}}{ }$ & $\begin{array}{l}\infty \\
\dot{v} \\
\dot{v}\end{array}$ & $\begin{array}{c}\infty \\
\substack{\infty\\
}\end{array}$ & \begin{tabular}{|l}
\multirow{y}{*}{} \\
\end{tabular} & $\begin{array}{l}\alpha \\
\stackrel{\alpha}{\sigma}\end{array}$ & $\bar{g}$ & $\begin{array}{l}m \\
\tilde{m} \\
m\end{array}$ & $\frac{m}{\sigma}$ & $\approx$ & $\overline{i n}$ & $\stackrel{n}{n !}$ & $\check{\mathcal{F}}$ & $\begin{array}{l}\tilde{\infty} \\
\stackrel{m}{m}\end{array}$ \\
\hline$₹$ & if & \begin{tabular}{l|}
+ \\
$\infty$
\end{tabular} & 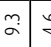 & 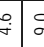 & $\infty_{\infty}^{\infty}$ & $\begin{array}{ll}m \\
\alpha\end{array}$ & $\begin{array}{ll}3 \\
2 \\
2\end{array}$ & 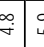 & $8 \infty$ & 恕 & m. & $:$ & $\begin{array}{c}\infty \\
\infty\end{array}$ & $\begin{array}{ll} \\
\vdots\end{array}$ & in & $\bar{\pi}$ & $\bar{\alpha}$ & $\stackrel{\infty}{\infty}$ & J. & $\begin{array}{l}\alpha \\
\infty\end{array}$ & $\stackrel{m}{F}$ & $\begin{array}{l}\alpha \\
\infty\end{array}$ & {$\left[\begin{array}{l}\infty \\
\infty \\
\infty\end{array}\right.$} \\
\hline$\Xi$ & 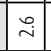 & $\underset{\sim}{J}$ & $\bar{\AA}$ & 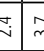 & $\stackrel{\vec{m}}{+}$ & $\underset{\sim}{\infty}$ & $\begin{array}{l}\text { ले } \\
\end{array}$ & 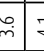 & $\bar{m}$ & $\underset{\sim}{\sim}$ & $\mid \begin{array}{ll}n \\
m\end{array}$ & 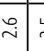 & $\hat{n}=$ & m & $\bar{i}$ & \begin{tabular}{|l|l|}
$m$ \\
$m$
\end{tabular} & m & $\begin{array}{l}\text { J } \\
\end{array}$ & ìm & $\bar{F}$ & $\tilde{N}$ & f & $\tilde{N}$ \\
\hline $\mathbb{S}$ & $\overline{\grave{\lambda}}$ & 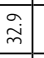 & 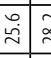 & \begin{tabular}{c|c}
5 \\
$\infty$
\end{tabular} & $\stackrel{\infty}{\beth}$ & ग. & $\stackrel{\infty}{\sim}$ & 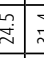 & $\dot{*}$ & $\overline{\text { D }}$ & 客 & 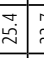 & 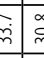 & \begin{tabular}{c|c}
$m$ \\
\end{tabular} & $\underset{\sim}{\mathbb{N}}$ & $\begin{array}{l}0 \\
\end{array}$ & \begin{tabular}{|l|}
$\infty$ \\
\\
\end{tabular} & $\stackrel{a}{a}$ & 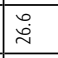 & 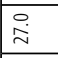 & गे & 渱 & $\stackrel{\vec{\sim}}{\tilde{N}}$ \\
\hline$\Xi$ & 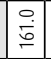 & 余 & $\overrightarrow{\dot{m}}$ & $\bar{c}$ & $\stackrel{\infty}{\dddot{m}}$ & 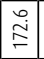 & 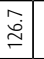 & \begin{tabular}{c}
2 \\
\hdashline \\
\hdashline
\end{tabular} & $\stackrel{b}{b}$ & \begin{tabular}{|l}
$\stackrel{\unlhd}{\Im}$ \\
$\Xi$
\end{tabular} & $\begin{array}{l}\stackrel{n}{m} \\
\underline{m}\end{array}$ & 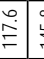 & 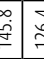 & $\dot{\infty}$ & $\stackrel{\mathbb{Z}}{\underline{\Xi}}$ & \begin{tabular}{l|l} 
\\
$\dot{z}$
\end{tabular} & 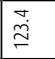 & $\overline{\underline{\sigma}}$ & $\begin{array}{l}\stackrel{\circ}{\dot{g}} \\
\end{array}$ & 总 & 总 & 㽞 & 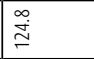 \\
\hline$\Xi$ & $\begin{array}{l}\widehat{a} \\
\underline{\underline{a}}\end{array}$ & 察 & $\bar{O}$ & 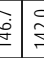 & 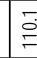 & 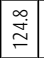 & : & 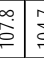 & 8 & 兽 & \begin{tabular}{|c|c|}
$\infty$ \\
$\alpha$ \\
$\alpha$
\end{tabular} & $\bar{\alpha} \bar{\alpha}$ & \begin{tabular}{c}
0 \\
\hdashline \\
\hdashline
\end{tabular} & ${ }_{m}^{m}$ & $\Phi_{\infty}$ & \begin{tabular}{|c|c|} 
\\
$\infty$ \\
$\infty$
\end{tabular} & 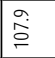 & 芯 & 总 & 尊 & 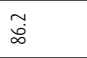 & : & 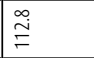 \\
\hline $\bar{z}$ & 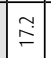 & \begin{tabular}{l|} 
\\
$\stackrel{\infty}{\infty}$ \\
\end{tabular} & $\bar{g}$ & $\tilde{c}$ & 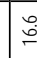 & \begin{tabular}{|l|l|}
\multicolumn{2}{|c|}{} \\
$\alpha$ \\
\end{tabular} & 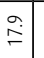 & 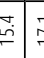 & $=3$ & 望 & \begin{tabular}{|l|l|}
\multicolumn{1}{|c|}{} & \\
$\circ$ &
\end{tabular} & $\stackrel{\infty}{\stackrel{\infty}{=}}$ & $\tilde{n}$ & $=5$ & $\underset{\sim}{\infty}$ & 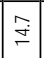 & 总 & 苛 & 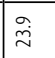 & 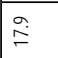 & 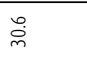 & 粕 & $\stackrel{ \pm}{ \pm}$ \\
\hline$\diamond$ & 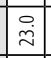 & $\begin{array}{l}\dot{m} \\
\dot{m}\end{array}$ & $\overline{i ்}$ & $\infty$ & 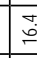 & \begin{tabular}{|l|l|} 
\\
\end{tabular} & 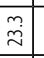 & 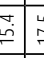 & $=7$ & $\stackrel{4}{d}$ & 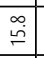 & बें & 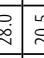 & 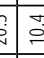 & $\stackrel{m}{m}$ & I & 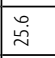 & 㕝 & 品 & $\grave{i}$ & 离 & $\stackrel{\sim}{\mathfrak{Z}}$ & $\stackrel{\infty}{i}$ \\
\hline 远 & $\vec{\infty}$ & $\stackrel{\circ}{\stackrel{\circ}{\leftrightarrows}}$ & $\stackrel{+}{\rightleftarrows}$ & $E$ & $\infty$ & 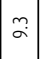 & $\stackrel{\infty}{\infty}$ & م. & $\%$ & $\infty$ & $\stackrel{1}{\therefore}$ & $\infty$ & $=$ & $=2$ & $\infty_{\infty}^{+}$ & $\stackrel{2}{\sim}$ & \& & $\bar{a}$ & $\infty$ & $\bar{\sigma}$ & ब & $\bar{\infty}$ & $\underset{\infty}{\infty}$ \\
\hline 올 & 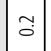 & $\therefore$ & \begin{tabular}{c|c}
$\tilde{O}$ & 2
\end{tabular} & 5 & $\bar{\sigma}$ & $\tilde{b}$ & $\approx$ & $\bar{b}$ & $5=$ & $\tilde{s}$ & $\approx$ & 3 & 5 & $5 \overline{0}$ & $\tilde{\sigma}$ & 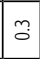 & $\ddot{g}$ & $\overline{0}$ & $\therefore$ & $\tilde{\sigma}$ & $\bar{o}$ & $\tilde{o}$ & '̄ \\
\hline$>$ & 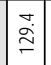 & 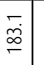 & $\underset{\sim}{\mathscr{a}}$ & & F & 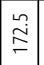 & 离 & $\begin{array}{l}\infty \\
\bar{E} \\
\bar{L}\end{array}$ & 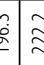 & 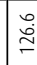 & $\stackrel{m}{\vec{m}}$ & 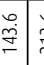 & $\begin{array}{l}\underbrace{\circ}_{0} \\
\end{array}$ & 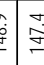 & $\begin{array}{l}\infty \\
\infty \\
\infty\end{array}$ & 童 & $\underset{\tilde{z}}{2}$ & 爱 & ळ. & $\begin{array}{l}\text { 总 } \\
\stackrel{-}{2}\end{array}$ & \begin{tabular}{l}
$\infty$ \\
$\underset{\sim}{\infty}$ \\
\multirow{\sim}{*}{}
\end{tabular} & 总 & 号 \\
\hline 气 & \begin{tabular}{|l|} 
\\
\end{tabular} & $\equiv$ & $\therefore:$ & 5 & a & $\stackrel{\circ}{-}$ & \begin{tabular}{l|l|l|}
$\infty$ \\
$\infty$
\end{tabular} & $\begin{array}{l}\infty \\
0 \\
0\end{array}$ & $=0$ & $\stackrel{\circ}{\sim}$ & oे & $:$ & $=:$ & $5 \div$ & $\div$ & $\stackrel{-}{-}$ & os & 8 & o & $\mp$ & 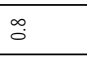 & $\stackrel{\infty}{\circ}$ & 8 \\
\hline : & 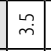 & $\stackrel{\sim}{\sim}$ & 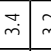 & $\bar{n} 2 \approx$ & $\bar{m}$ & $\tilde{m}$ & $\stackrel{\vec{m}}{m}$ & $\pi$ & $v_{m}$ & $\stackrel{\circ}{\sim}$ & $\stackrel{\vec{m}}{ }$ & $\tilde{m}$ & $\stackrel{\sim}{\sim}$ & 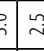 & $\stackrel{\sim}{\sim}$ & $\stackrel{2}{\sim}$ & $\begin{array}{l} \\
\text { m }\end{array}$ & $\tilde{m}$ & i & $\stackrel{i}{i}$ & $\bar{F}$ & $\bar{m}$ & r \\
\hline$\stackrel{8}{ \pm 0}$ & $m$ & $\simeq$ & $8=$ & $=a$ & & $\simeq$ & $\therefore$ & 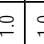 & $=7$ & $F$ & $=$ & \pm 0 & \begin{tabular}{l|l}
$\infty$ \\
0
\end{tabular} & 12 & $=$ & $\stackrel{m}{\sim}$ & $m$ & $\stackrel{\circ}{\circ}$ & $\bar{F}$ & $\bar{F}$ & $\stackrel{8}{\circ}$ & 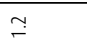 & I8 \\
\hline 욜 & $\begin{array}{c} \\
\\
\end{array}$ & 它 & $\bar{\infty}$ & $\begin{array}{l}0 \\
0\end{array}$ & & 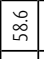 & $\begin{array}{c}1 \\
\vdots\end{array}$ & $\begin{array}{l}0 \\
\end{array}$ & $\overbrace{0}^{\circ}$ & $\stackrel{\infty}{\vdots}$ & \begin{tabular}{|c|}
2 \\
\end{tabular} & 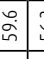 & $\begin{array}{c}0 \\
\end{array}$ & $\begin{array}{c}n \\
0\end{array}$ & م. & $\frac{9}{\bar{b}}$ & \begin{tabular}{|l|}
5 \\
5
\end{tabular} & 怘 & $\frac{m}{b}$ & 永 & 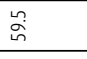 & $\frac{m}{b}$ & 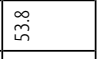 \\
\hline$\frac{0}{4} \bar{a}$ & $\approx$ & 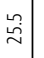 & $\bar{\pi}$ & 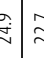 & $g$ & $\stackrel{\infty}{\underset{\sim}{\sim}}$ & $\nexists$ & iे & 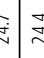 & $\frac{\alpha}{\bar{\tau}}$ & $\stackrel{\Re}{\approx}$ & $\stackrel{\sim}{\sim}$ & $\begin{array}{c}: \\
\sim\end{array}$ & 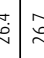 & $\stackrel{\infty}{\infty}$ & $\stackrel{\approx}{\approx}$ & $\underset{\dot{I}}{ \pm}$ & 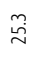 & 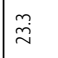 & $\overline{\mathbf{z}}$ & 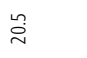 & $\stackrel{\infty}{\sim}$ & $\stackrel{\Xi}{\approx}$ \\
\hline 올 & $\stackrel{m}{-2}$ & $=$ & $\begin{array}{ll}0.7 \\
\circ\end{array}$ & $=7$ & & $\stackrel{.}{-}$ & $\stackrel{0}{\circ}$ & $\begin{array}{l}\infty \\
0\end{array}$ & $=3$ & $\cong$ & $\simeq$ & $\bar{~} \equiv$ & 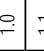 & $=9$ & a & g. & $\stackrel{\circ}{-}$ & $\stackrel{-}{-}$ & ò & $=$ & $\bar{i}$ & $\stackrel{\infty}{\infty}$ & 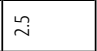 \\
\hline 要这 & $\cong$ & ó & $\cong$ & $=7$ & & $=$ & \pm 1 & 8 & $=9$ & $\stackrel{m}{?}$ & $\simeq$ & $\stackrel{2}{2}:$ & : & $=0$ & o & $\stackrel{.}{\mp}$ & $\tilde{N}$ & go & $\mp$ & $\cong$ & 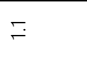 & $\cong$ & $\stackrel{\infty}{-}$ \\
\hline$\infty$ & $\stackrel{\tilde{I}}{I}$ & 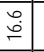 & \begin{tabular}{c|c}
$\circ$ \\
\end{tabular} & 車 & & $\underset{\substack{ \pm \pm}}{ }$ & 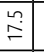 & 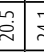 & 59 & 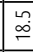 & : & 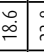 & $\underset{\sim}{\infty})$ & 5 & $\bar{F}_{\dot{F}}$ & $\begin{array}{l}\infty \\
\stackrel{\infty}{\infty}\end{array}$ & $\tilde{Z}$ & di & 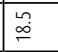 & $\stackrel{\varpi}{\varpi}$ & $\stackrel{\Phi}{\Phi}$ & $\stackrel{\Phi}{\stackrel{\Xi}{g}}$ & $\stackrel{\circ}{\circ}$ \\
\hline$=$ & 官 & $\begin{array}{l}\infty \\
\infty \\
\infty\end{array}$ & ले & 85 & & \begin{tabular}{|l|}
$\infty$ \\
$\vdots$ \\
\end{tabular} & 3 & 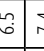 & 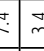 & $\stackrel{m}{r}$ & \begin{tabular}{|l|} 
\\
5 \\
\end{tabular} & $m ?$ & 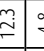 & \begin{tabular}{c|c} 
\\
\end{tabular} & 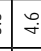 & 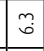 & 吉 & 8 & 占 & g) & $\stackrel{\text { İ }}{\stackrel{2}{*}}$ & $\stackrel{9}{9}$ & $\stackrel{\infty}{\stackrel{\infty}{\infty}}$ \\
\hline 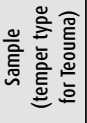 & & & & & & & & & & & & & & & & & 言 & & 离 & 辛 & 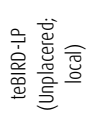 & 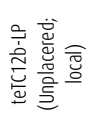 & \\
\hline 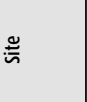 & & & & & & & & & & & & & & & & & & & 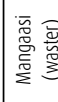 & & & & 总 \\
\hline
\end{tabular}




\begin{tabular}{|c|c|c|c|c|c|c|c|c|c|c|c|c|c|}
\hline$=$ & $F$ & $\mid$\begin{tabular}{|l|}
$\infty$ \\
0
\end{tabular} & $\tilde{v}$ & $\stackrel{\rho}{\tau}$ & $\stackrel{\infty}{=}$ & $\overline{\mathrm{N}}$ & $\stackrel{i}{i}$ & $\hat{0}$ & 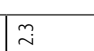 & 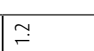 & $\stackrel{2}{\sim}$ & i & : \\
\hline$E$ & $\stackrel{10}{1}$ & $\stackrel{\rho}{\tau}$ & 8 & F & 8 & $\stackrel{\infty}{\infty}$ & F & 10 & 官 & 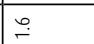 & F & $\infty_{\infty}^{\infty}$ & $\stackrel{8}{1}$ \\
\hline a & 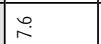 & so & 吕 & \begin{tabular}{l}
$l o g$ \\
\hdashline
\end{tabular} & $\underset{\substack{\infty \\
\sim}}{2}$ & $\vec{m}$ & I & $F$ & {$\left[\begin{array}{l}5 \\
\end{array}\right.$} & $\approx$ & I & $\stackrel{\Omega}{i}$ & $f$ \\
\hline$\underline{x}$ & $\tilde{m}$ & $i$ & $\infty$ & $f$ & or & $\stackrel{n}{\sim}$ & $f$ & $\bar{N}$ & 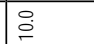 & $\tilde{m}$ & n & in & $\bar{N}$ \\
\hline$\stackrel{\rho}{\geqslant}$ & \begin{tabular}{|l|l|} 
\\
\end{tabular} & $\stackrel{\infty}{\sim}$ & $\bar{i}$ & $\overline{\bar{N}}$ & $\dot{i}$ & $\overline{\mathrm{N}}$ & I & $\stackrel{m}{\rho}$ & $\stackrel{2}{\square}$ & \begin{tabular}{|l|l|} 
\\
\hdashline
\end{tabular} & i & $\stackrel{2}{2}$ & $\dot{i}$ \\
\hline$=$ & 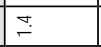 & $\stackrel{\mid}{\circ}$ & $\stackrel{\infty}{-\infty}$ & g & $=$ & \% & $\overline{\mathrm{i}}$ & $\cong$ & $m$ & $\underset{\square}{J}$ & $\tilde{\lambda}$ & $\stackrel{\mid \Xi}{\mp}$ & $\stackrel{i}{i}$ \\
\hline$a$ & $\stackrel{i}{i}$ & $\pi$ & $\stackrel{m}{m}$ & I & 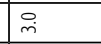 & $\bar{m}$ & 年 & $\bar{i}$ & $\bar{i}$ & $\bar{i}$ & 年 & $\stackrel{n}{2}$ & $\bar{m}$ \\
\hline $\mathscr{S}$ & $\stackrel{\infty}{=}$ & $\stackrel{i}{i}$ & $\bar{m}$ & $\underset{\sim}{\infty}$ & $\vec{i}$ & $m$ & 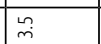 & $\bar{i}$ & $\bar{N}$ & 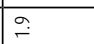 & $F$ & $\left.\right|^{\prime}$ & $\stackrel{\sim}{\sim}$ \\
\hline$\Xi$ & $\stackrel{\rho}{=}$ & 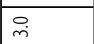 & $\dot{q}$ & $\underset{m}{\mid r}$ & 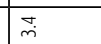 & P & f & $\stackrel{2}{\sim}$ & $\stackrel{2}{\sim}$ & $\stackrel{i}{i}$ & 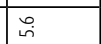 & $\tilde{m}$ & in \\
\hline $\bar{z}$ & $m$ & $\stackrel{\square}{\rightleftarrows}$ & $\Xi$ & 픈 & $\stackrel{p}{q}$ & $\stackrel{I}{I}$ & 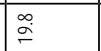 & $\alpha_{\infty}^{\infty}$ & $\stackrel{P}{F}$ & 齐 & $\bar{j}$ & 电 & 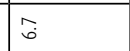 \\
\hline s & $\hat{\dot{\rho}}$ & $\tilde{\tilde{I}}$ & \begin{tabular}{|l|}
$\vec{a}$ \\
$\dot{m}$
\end{tabular} & $\begin{array}{l} \\
\dot{i}\end{array}$ & $\begin{array}{l}\overrightarrow{2} \\
\text { in }\end{array}$ & $\tilde{\tilde{I}}$ & 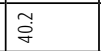 & $\dot{\alpha}$ & $\stackrel{m}{i}$ & 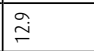 & \begin{tabular}{|l|l|}
$\dot{\sigma o g}$ \\
\end{tabular} & $\frac{\vec{m}}{m}$ & $\bar{\alpha}$ \\
\hline$\Xi$ & 5 & $\bar{\sigma}$ & $\Xi$ & 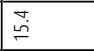 & \begin{tabular}{|l|}
$\dot{m}$ \\
$\dot{m}$
\end{tabular} & 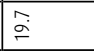 & \begin{tabular}{|l}
$\circ$ \\
$\stackrel{\circ}{\circ}$
\end{tabular} & $\bar{\infty}$ & 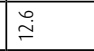 & ฮิ & $\frac{\partial}{\bar{\lambda}}$ & $\stackrel{m}{R}$ & $m$ \\
\hline$\cong$ & \begin{tabular}{|l|}
\multirow{2}{*}{} \\
for
\end{tabular} & $\mid$\begin{tabular}{|l|}
$\tilde{\infty}$ \\
$\infty$ \\
$\infty$
\end{tabular} & \begin{tabular}{|l|}
\multicolumn{2}{|r}{} \\
合
\end{tabular} & 焉 & 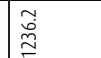 & 势 & 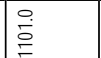 & 总 & 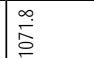 & \begin{tabular}{|l|}
$\tilde{2}$ \\
aू
\end{tabular} & 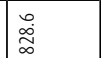 & \begin{tabular}{|l|} 
\\
\end{tabular} & 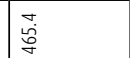 \\
\hline$\approx$ & \begin{tabular}{|l|l}
\multicolumn{2}{|l}{} \\
\end{tabular} & $m$ & \begin{tabular}{|l|l|}
$\infty$ \\
\end{tabular} & $\stackrel{\rho}{\tau}$ & $\tilde{m}$ & $\vec{m}$ & $\bar{\lambda}$ & i & $\stackrel{2}{\sim}$ & $\cong$ & \begin{tabular}{|l|}
$\nsubseteq$ \\
\end{tabular} & $\bar{F}$ & $\bar{\lambda}$ \\
\hline 5 & $\stackrel{I}{\perp}$ & $F$ & $\stackrel{n}{m}$ & 电 & \% & $\stackrel{\circ}{m}$ & $\pi$ & $\begin{array}{ll} \\
\end{array}$ & iे & 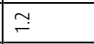 & $m$ & 喟 & $\cong$ \\
\hline$\stackrel{\circ}{\Sigma}$ & ó & $\underset{\sim}{\stackrel{\infty}{\sim}}$ & $\stackrel{\text { q }}{2}$ & 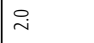 & $\approx$ & $\stackrel{a}{m}$ & $\stackrel{\sim}{i}$ & $\cong$ & $\underset{m}{m}$ & $\stackrel{\infty}{\infty}$ & $\stackrel{\sim}{\sim}$ & $\underbrace{\circ}_{\circ}$ & à \\
\hline$\hat{z}$ & $\stackrel{n}{i}$ & i & \begin{tabular}{|l|} 
\\
\end{tabular} & $\begin{array}{l} \\
\end{array}$ & \begin{tabular}{|l|l|}
$\infty$ \\
\end{tabular} & $\stackrel{n}{n}$ & 年 & $\stackrel{i}{i}$ & \begin{tabular}{|l|l|}
$\infty$ \\
\end{tabular} & $\stackrel{n}{i}$ & \begin{tabular}{|l|}
$\infty$ \\
nin \\
\end{tabular} & in & \begin{tabular}{|l|l|}
$\infty$ \\
\end{tabular} \\
\hline$\approx$ & 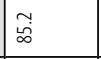 & $\stackrel{2}{i}$ & 总 & 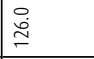 & \begin{tabular}{|l|l} 
\\
.0 \\
0
\end{tabular} & $\frac{m^{2}}{i}$ & 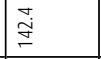 & \begin{tabular}{|l}
$\infty$ \\
0 \\
0
\end{tabular} & \begin{tabular}{|l}
$\infty$ \\
㐫 \\
N.
\end{tabular} & $\check{\infty}$ & 桀 & \begin{tabular}{|l|l}
$\infty$ \\
0 \\
0
\end{tabular} & 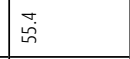 \\
\hline$>$ & \begin{tabular}{|l|}
\multirow{0}{*}{} \\
\end{tabular} & $\stackrel{m}{F}$ & \begin{tabular}{|l|} 
\\
\end{tabular} & \begin{tabular}{|l|}
$\underline{0}$ \\
\end{tabular} & $\underline{\underline{m}}$ & $\exists$ & \begin{tabular}{|l|}
$\infty$ \\
$\stackrel{\infty}{\infty}$
\end{tabular} & \begin{tabular}{|l|}
$\infty$ \\
$\infty$
\end{tabular} & \begin{tabular}{|l|l|}
$\infty$ \\
$\stackrel{0}{\circ}$
\end{tabular} & $\begin{array}{l}\text { Oे } \\
\end{array}$ & \begin{tabular}{|l|} 
\\
\end{tabular} & 녕 & \begin{tabular}{|l|}
$\infty$ \\
$\stackrel{\rho}{\rho}$ \\
\end{tabular} \\
\hline$=$ & $\mid$ & $\mid \begin{array}{l}0 \\
\vdots \\
\vdots \\
6\end{array}$ & 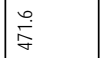 & $\mid \begin{array}{l}\infty \\
\vdots \\
\vdots \\
\end{array}$ & 离 & 雍 & 命 & 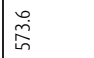 & 前 & 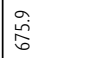 & 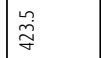 & 产 & 京 \\
\hline$\stackrel{\propto}{\propto}$ & $\bar{m}$ & 番 & $\stackrel{\sim}{\sim}$ & $\bar{f}$ & 总 & $\stackrel{\substack{\xi \\
\dot{\gamma}}}{ }$ & in & $\bar{\rho}$ & $\tilde{F}$ & 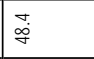 & 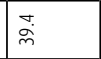 & ذి & 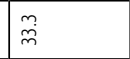 \\
\hline z & \begin{tabular}{|c|} 
Oे \\
\end{tabular} & in & $\underset{\exists}{\not}$ & $\begin{array}{l}\text { gi } \\
\end{array}$ & $\tilde{\tilde{O}}$ & 枲 & $\begin{array}{l}\infty \\
\infty\end{array}$ & نْ & 吕 & $\bar{\Phi}$ & $\stackrel{\infty}{\stackrel{\infty}{\rightleftharpoons}}$ & $\tilde{m}$ & $\frac{m}{q}$ \\
\hline 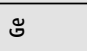 & $\stackrel{2 N}{i}$ & \begin{tabular}{|l|l|} 
\\
$\stackrel{i}{i}$
\end{tabular} & \begin{tabular}{|l|} 
\\
$m$
\end{tabular} & $\bar{m}$ & \begin{tabular}{|l|} 
m \\
\end{tabular} & \begin{tabular}{|l|}
$\infty$ \\
+ \\
+
\end{tabular} & \begin{tabular}{|l|l|}
$\infty$ \\
in
\end{tabular} & 官 & 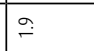 & $\stackrel{9}{2}$ & $\bar{m}$ & \begin{tabular}{|l|}
$\infty$ \\
$\infty$
\end{tabular} & $\frac{\rho}{2}$ \\
\hline$\pi$ & $\check{i}$ & 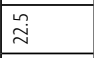 & 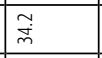 & $\check{i}$ & 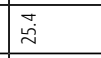 & \begin{tabular}{|l|l|}
0 \\
\end{tabular} & $\begin{array}{l}m \\
I \\
I\end{array}$ & $\tilde{\varkappa}$ & $\bar{a}$ & $\underset{i}{\infty}$ & \begin{tabular}{|l|}
$\infty$ \\
$\infty$
\end{tabular} & $\stackrel{9}{2}$ & \begin{tabular}{|l|}
$\stackrel{\mathscr{\Omega}}{\infty}$ \\
\end{tabular} \\
\hline$\approx$ & 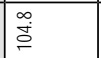 & 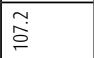 & 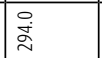 & 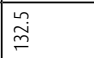 & 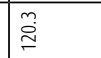 & 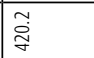 & 点 & 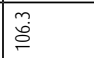 & 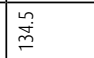 & $\overline{\underline{m}}$ & 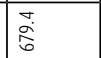 & 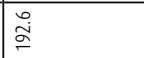 & 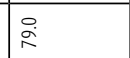 \\
\hline כ & \begin{tabular}{|l|}
$\stackrel{\infty}{\infty}$ \\
$\dot{\infty}$
\end{tabular} & 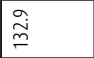 & mon & 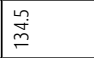 & 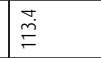 & 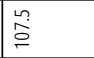 & $\begin{array}{l}\infty \\
\alpha \\
\alpha\end{array}$ & \begin{tabular}{|l|} 
\\
\\
\end{tabular} & $\begin{array}{l} \\
\end{array}$ & 葛 & 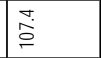 & חె & 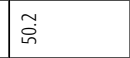 \\
\hline $\bar{z}$ & $\stackrel{\infty}{\sim}$ & \begin{tabular}{|l}
$\infty$ \\
$\stackrel{\infty}{\sim}$
\end{tabular} & $\Xi$ & ì & $\stackrel{m}{\mathscr{2}}$ & $\underset{I}{q}$ & $\underset{f}{\stackrel{f}{m}}$ & $\begin{array}{l}\infty \\
\infty \\
\infty\end{array}$ & $\stackrel{\Perp}{\simeq}$ & 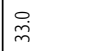 & 总 & 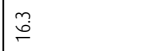 & 家 \\
\hline$\diamond$ & 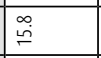 & \begin{tabular}{|l|}
\multirow{2}{*}{} \\
\end{tabular} & \begin{tabular}{|c|}
$\tilde{i}$ \\
\end{tabular} & $\approx$ & \begin{tabular}{|l|}
8 \\
\end{tabular} & \begin{tabular}{|l|}
$\vec{m}$ \\
\end{tabular} & \begin{tabular}{|l|}
\multirow{\infty}{\prime}{} \\
\end{tabular} & $\overline{\tilde{\nu}}$ & \begin{tabular}{|l|}
\multicolumn{2}{|l|}{} \\
$\infty$
\end{tabular} & $\underline{m}$ & \begin{tabular}{|l|l}
$m$ \\
\end{tabular} & \begin{tabular}{|l|}
$\sigma$ \\
\end{tabular} & $\overline{\dot{i}}$ \\
\hline बa & F & $\stackrel{\infty}{\stackrel{\infty}{m}}$ & \begin{tabular}{|}
$\infty$ \\
$\dot{\mid}$ \\
\end{tabular} & a & $\stackrel{2}{2}$ & $\overbrace{j}$ & $\stackrel{2}{2}$ & 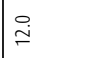 & $\infty$ & $\stackrel{\circ}{\circ}$ & 㒸 & $\stackrel{m}{m}$ & $\widehat{\infty}$ \\
\hline 올 & $\overline{0}$ & $\overline{0}$ & $\tilde{o}$ & $\overline{0}$ & $\overline{0}$ & a & $\overline{0}$ & $\overline{0}$ & $\overline{0}$ & $\overline{0}$ & nू & 5 & $\therefore$ \\
\hline$>$ & $\underset{\partial}{\tilde{D}}$ & 䀦 & $\mid \begin{array}{l}\partial \\
\dot{\rho} \\
\bar{\rho}\end{array}$ & 高 & 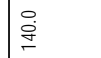 & $\begin{array}{l}\infty \\
\tilde{\tilde{\sigma}} \\
\end{array}$ & 品 & $\stackrel{n}{\stackrel{2}{n}}$ & 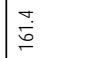 & 岕 & 产 & $\underset{m}{g}$ & 文 \\
\hline 을 & \begin{tabular}{|l|}
$\infty$ \\
$\infty$ \\
\end{tabular} & \begin{tabular}{|l|} 
\\
$\infty$ \\
$\infty$
\end{tabular} & $\bar{i}$ & \begin{tabular}{|l|} 
\\
\end{tabular} & \begin{tabular}{|l|} 
\\
\end{tabular} & $\tilde{m}$ & a & $\begin{array}{l}\infty \\
\infty \\
\end{array}$ & $\equiv$ & $\begin{array}{l}\infty \\
\infty \\
\infty\end{array}$ & f & $\because$ & \begin{tabular}{|l|} 
\\
- \\
\end{tabular} \\
\hline 。্ర & 禺 & $\stackrel{m}{m}$ & \begin{tabular}{|l|}
$\vec{m}$ \\
\end{tabular} & 讨 & o & $\vec{m}$ & $\tilde{m}$ & $\bar{m}$ & i & Pi & $\underset{\sim}{\infty}$ & \begin{tabular}{|l|} 
in \\
\end{tabular} & $\vec{m}$ \\
\hline$\stackrel{0}{0}$ & $\begin{array}{l} \\
\end{array}$ & $\stackrel{m}{2}$ & $\mp$ & \begin{tabular}{|l|} 
\\
\\
\hdashline
\end{tabular} & \pm & 告 & 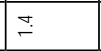 & aे & $\stackrel{\circ}{\div}$ & 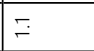 & ${ }_{0}^{\circ}$ & $\begin{array}{l}\infty \\
0 \\
0\end{array}$ & $\begin{array}{ll} \\
\end{array}$ \\
\hline 行 & 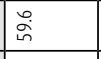 & 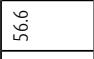 & \begin{tabular}{|l|l}
$m$ \\
$n$
\end{tabular} & 点 & 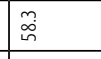 & \begin{tabular}{|l|}
5 \\
$y$
\end{tabular} & \begin{tabular}{|l|l}
$\frac{2}{5}$ \\
5
\end{tabular} & 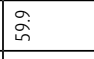 & \begin{tabular}{|l|l|}
\multirow{2}{n}{} \\
\end{tabular} & 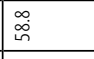 & \begin{tabular}{|l|l|}
$y$ \\
$y$
\end{tabular} & f & \begin{tabular}{|l|l}
$\tilde{\sigma}$ \\
\end{tabular} \\
\hline$\frac{0}{\alpha}$ & 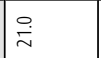 & 咅 & $\ddot{\sim}$ & $\stackrel{\sim}{\underset{N}{*}}$ & 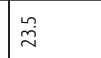 & i্ & $\stackrel{\infty}{\sim}$ & i & i & 品 & 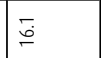 & î̀ & 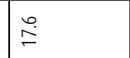 \\
\hline 올 & $\infty$ & $\mp$ & o & $\begin{array}{l}\infty \\
0 \\
0\end{array}$ & $\because$ & $\stackrel{+}{\circ}$ & $\begin{array}{l}\infty \\
\infty \\
\infty\end{array}$ & 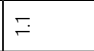 & 号 & i & $\bar{i}$ & $\stackrel{2}{\square}$ & I \\
\hline 屃 & $\stackrel{m}{=}$ & $\stackrel{\infty}{-}$ & ô & $\stackrel{\infty}{\perp}$ & \begin{tabular}{|l|}
$\infty$ \\
-
\end{tabular} & : & 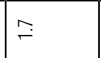 & $\cong$ & aे & a & I & o & $\stackrel{2}{i}$ \\
\hline$\infty$ & \begin{tabular}{|l|} 
वे \\
\end{tabular} & 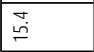 & $\nexists$ & I & $\bar{i}$ & I & $\tilde{\omega}$ & \begin{tabular}{|l}
$m$ \\
m
\end{tabular} & \begin{tabular}{|l|}
$\infty$ \\
$\stackrel{\infty}{\circ}$
\end{tabular} & $\frac{\vec{T}}{\pi}$ & $\begin{array}{l} \\
\end{array}$ & ‡્ & $\underline{\underline{g}}$ \\
\hline $\bar{z}$ & \begin{tabular}{|l|} 
\\
$\stackrel{f}{\infty}$ \\
\end{tabular} & 5 & \begin{tabular}{|l|}
5 \\
मे
\end{tabular} & $\begin{array}{l}n \\
\infty \\
\infty\end{array}$ & $\begin{array}{l}\infty \\
\infty\end{array}$ & $\bar{m}$ & $\infty$ & $\bar{\infty}$ & \begin{tabular}{|l|} 
\\
\end{tabular} & $\stackrel{I}{ \pm}$ & \begin{tabular}{|l|}
$\infty$ \\
$\infty$
\end{tabular} & অ & 2 \\
\hline 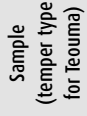 & $\mid$ & 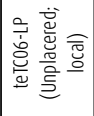 & 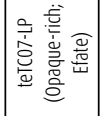 & 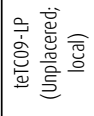 & 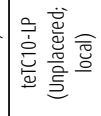 & 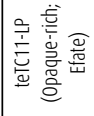 & 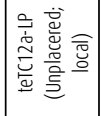 & 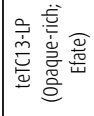 & 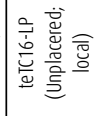 & 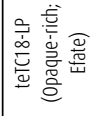 & 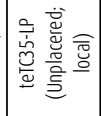 & 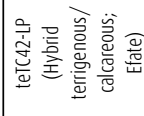 & 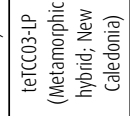 \\
\hline$\stackrel{2}{\tilde{\Sigma}}$ & 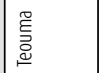 & 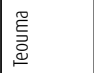 & 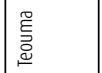 & 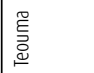 & 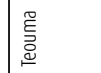 & 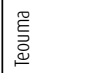 & 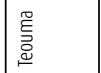 & 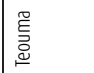 & 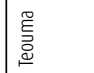 & $\begin{array}{l}\text { 总 } \\
\text { 掌 }\end{array}$ & 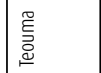 & \begin{tabular}{|l|} 
墨 \\
兑
\end{tabular} & 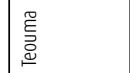 \\
\hline
\end{tabular}




\begin{tabular}{|c|c|c|c|c|c|c|c|c|c|c|}
\hline 0 & $\stackrel{2}{i}$ & $\stackrel{2}{2}$ & 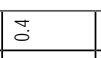 & $\cong$ & $\stackrel{i}{i}$ & \begin{tabular}{|l}
$\circ$ \\
\end{tabular} & $\begin{array}{ll}n \\
b \\
0\end{array}$ & $\stackrel{m}{m}$ & $\cong$ & \begin{tabular}{|l|} 
\\
\end{tabular} \\
\hline$E$ & $\stackrel{\circ}{\circ}$ & $\bar{\gamma}$ & g. & $\stackrel{\infty}{-}$ & $m$ & $\stackrel{2}{\circ}$ & g. & g & o & $\bar{m}$ \\
\hline a & \begin{tabular}{|l|}
$g$ \\
\end{tabular} & $\tilde{\omega}$ & $\begin{array}{l} \\
\end{array}$ & 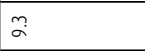 & 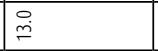 & $\stackrel{\beth}{\stackrel{I}{\sim}}$ & $m$ & 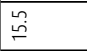 & 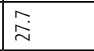 & $\Xi$ \\
\hline 至 & $\stackrel{\rho}{\rho}$ & 我 & స & $\stackrel{\sim}{\sim}$ & $\overrightarrow{\mathrm{i}}$ & $\stackrel{m}{m}$ & $\bar{i}$ & ì & $\stackrel{2}{2}$ & $\stackrel{\infty}{m}$ \\
\hline 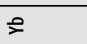 & $\tilde{\lambda}$ & $\stackrel{\infty}{\sim}$ & $\vec{i}$ & \begin{tabular}{|l|l|}
0 \\
\end{tabular} & $g$ & 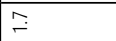 & $\approx$ & $\bar{i}$ & 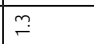 & $\tilde{i}$ \\
\hline \pm & \begin{tabular}{|l|}
4 \\
\end{tabular} & $\bar{i}$ & $\stackrel{g}{g}$ & !n & 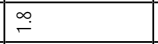 & 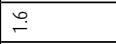 & $\begin{array}{ll}0 \\
\end{array}$ & $\bar{i}$ & $\underline{m}$ & $\stackrel{g}{\sigma}$ \\
\hline $\bar{a}$ & 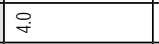 & \begin{tabular}{|l|l}
+9 \\
\end{tabular} & ì & $\underset{\sim}{\infty}$ & iे & $\bar{I}$ & $\stackrel{2}{i}$ & g & $\bar{i}$ & $\underset{\sim}{\infty}$ \\
\hline$\Xi$ & f & F & I & $\dot{\mathcal{H}}$ & $\bar{i}$ & $\stackrel{\infty}{\check{\infty}}$ & $\bar{i}$ & 齐 & $\stackrel{2}{2}$ & I \\
\hline$\Xi$ & \begin{tabular}{|l|}
7 \\
\end{tabular} & ถึ่ & $\stackrel{\mathrm{I}}{\mathrm{I}}$ & $\stackrel{J}{\mathrm{i}}$ & $\underset{\sim}{\infty}$ & $\begin{array}{ll}0 \\
\stackrel{-}{\circ}\end{array}$ & in & $\overline{s i n}$ & 酋 & I \\
\hline $\bar{z}$ & 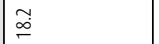 & $\overline{\tilde{z}}$ & $\approx$ & $\hat{a}$ & $\bar{\Xi}$ & fi & $\stackrel{2}{2}$ & $\stackrel{\circ}{g}$ & $\cong$ & g \\
\hline ๘ & $\tilde{z}$ & 集 & $\stackrel{\infty}{\stackrel{\infty}{\rightleftharpoons}}$ & $\stackrel{n}{\beth}$ & $\bar{i}$ & $\bar{\sigma}$ & 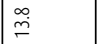 & $\overline{\vec{J}}$ & $\stackrel{i}{i}$ & 吕 \\
\hline$\Xi$ & $\bar{g}$ & $\stackrel{n}{2}$ & 文 & $\stackrel{\infty}{\sim}$ & $\stackrel{\circ}{\stackrel{\circ}{\circ}}$ & $\bar{i}$ & F & $\stackrel{I}{I}$ & 点 & 㛡 \\
\hline$\cong$ & ڤे & 产 & $\stackrel{n}{2}$ & 足 & 店 & 号 & ळ్ & 검 & 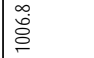 & $\mid \begin{array}{l}\stackrel{\circ}{\infty} \\
\frac{\infty}{\infty}\end{array}$ \\
\hline$\Xi$ & $\vec{i}$ & $\vec{\rho}$ & $\bar{m}$ & $\bar{m}$ & $\frac{9}{8}$ & $\overrightarrow{\mathrm{i}}$ & $\stackrel{\infty}{\infty}$ & $\tilde{\text { I }}$ & 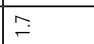 & $\begin{array}{ll}\circ \\
\end{array}$ \\
\hline 5 & $\underline{m}$ & $m$ & $\stackrel{P}{P}$ & 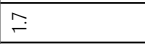 & 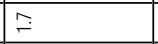 & $\stackrel{?}{\circ}$ & 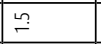 & $\pi$ & $\underset{\sim}{\infty}$ & $\cong$ \\
\hline$\stackrel{\circ}{\gtrless}$ & 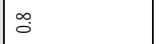 & $\stackrel{\sim}{\sim}$ & : & 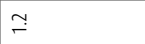 & $\stackrel{ \pm}{\simeq}$ & $\stackrel{\infty}{\infty}$ & $\hat{0}$ & m & 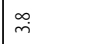 & \begin{tabular}{|l|l}
2 \\
9
\end{tabular} \\
\hline $\bar{z}$ & $\dot{i}$ & 吕 & $\bar{i}$ & i & 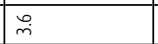 & $\tilde{I}$ & $\mp$ & i & $\bar{s}$ & 2 \\
\hline$\pi$ & 芛 & 总 & $\stackrel{i}{i}$ & 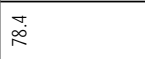 & $\underset{\sim}{\infty}$ & $\bar{Z}$ & 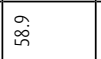 & \begin{tabular}{|l|}
$\dot{\infty}$ \\
$\dot{\infty}$
\end{tabular} & \begin{tabular}{|l|}
$\partial$ \\
$\partial$ \\
\end{tabular} & \begin{tabular}{|l|}
$\stackrel{\circ}{\circ}$ \\
\end{tabular} \\
\hline$>$ & 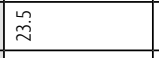 & $\stackrel{\circ}{\stackrel{\circ}{~}}$ & $\bar{\Xi}$ & $\cong$ & \begin{tabular}{|l|}
$\circ$ \\
\end{tabular} & $\overline{\underline{S}}$ & $\bar{\Xi}$ & \begin{tabular}{|l|}
$\stackrel{\infty}{\check{g}}$ \\
\end{tabular} & \begin{tabular}{|l|l}
$g$ \\
$\stackrel{g}{g}$
\end{tabular} & \begin{tabular}{|l|}
$g$ \\
$口$
\end{tabular} \\
\hline$=$ & 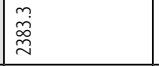 & 商 & ఏి & 今े & 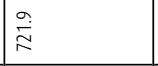 & 足 & 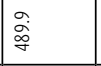 & 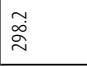 & 总 & 产 \\
\hline 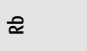 & ల్ & $\underset{m}{\stackrel{m}{m}}$ & $\stackrel{n}{n}$ & \begin{tabular}{|l|l} 
\\
8 \\
\end{tabular} & 苟 & 苂 & $\exists$ & $\frac{m}{m}$ & 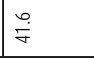 & 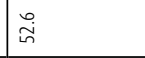 \\
\hline 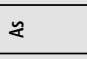 & \begin{tabular}{|l|l|}
$\infty$ \\
$\stackrel{\infty}{\circ}$
\end{tabular} & $\begin{array}{l}\sigma \\
\text { in } \\
\end{array}$ & $\overline{i n}$ & \begin{tabular}{|l|l|}
$a$ \\
\end{tabular} & $\begin{array}{ll} \\
\end{array}$ & \begin{tabular}{|l|} 
\\
\end{tabular} & $\begin{array}{ll}\infty \\
\infty \\
\sigma_{0}\end{array}$ & $\begin{array}{l}\text { gे } \\
\end{array}$ & $\tilde{\Omega}$ & \begin{tabular}{|l|} 
\\
\end{tabular} \\
\hline 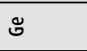 & \begin{tabular}{|l|}
$a$ \\
0
\end{tabular} & $\pi$ & $\stackrel{2}{2}$ & $m$ & \begin{tabular}{|l|l} 
\\
\end{tabular} & $\begin{array}{l}\text { o } \\
\end{array}$ & $m$ & $\infty$ & $\pi$ & 8 \\
\hline$\Xi$ & $\tilde{\tilde{\chi}}$ & 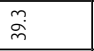 & 点 & 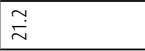 & \begin{tabular}{|l}
2 \\
$\bar{\lambda}$
\end{tabular} & \begin{tabular}{|l|}
$g$ \\
mi
\end{tabular} & वें & 古 & 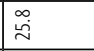 & \begin{tabular}{|l|l|}
\multirow{2}{\infty}{} \\
$\stackrel{\infty}{\sim}$
\end{tabular} \\
\hline$\approx$ & 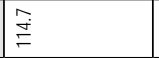 & 总 & $\stackrel{+}{\cong}$ & $\begin{array}{l}\stackrel{\circ}{\circ} \\
\stackrel{2}{\prime}\end{array}$ & 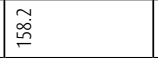 & go & 然 & 总 & 总 & 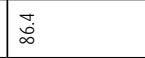 \\
\hline$\Xi$ & $\underset{\sigma}{\sigma}$ & $\stackrel{\vec{\Xi}}{\stackrel{I}{\Xi}}$ & 范 & $\stackrel{m}{\underline{E}}$ & $\stackrel{\Xi}{\Xi}$ & $\bar{E}$ & $\stackrel{m}{g}$ & 曽 & 放 & 京 \\
\hline $\bar{z}$ & $\begin{array}{l}\text { హ. } \\
\end{array}$ & $\bar{z}$ & 怘 & 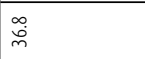 & \begin{tabular}{|l|}
8 \\
8 \\
\end{tabular} & 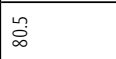 & 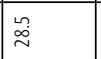 & $\tilde{j}$ & $\Xi$ & के \\
\hline 8 & $\begin{array}{l}a \\
\text { a }\end{array}$ & $\begin{array}{ll}0 \\
\end{array}$ & ্ָলি & $\underline{\Omega}$ & $\stackrel{\partial}{\tilde{i}}$ & 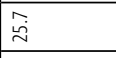 & İ & 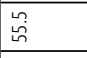 & $\begin{array}{l} \\
\end{array}$ & \begin{tabular}{|l|} 
\\
$\infty$
\end{tabular} \\
\hline ชิ & $\stackrel{\infty}{\propto}$ & 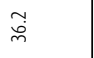 & $\stackrel{\varrho}{\longleftarrow}$ & $\stackrel{\circ}{\circ}$ & $\bar{a}$ & $\stackrel{?}{\rightleftarrows}$ & $\stackrel{n}{=}$ & $\underset{\sim}{\stackrel{\sim}{~}}$ & $\stackrel{8}{9}$ & 市 \\
\hline 올 & 5 & $\begin{array}{l}0 \\
0\end{array}$ & $\overline{0}$ & 5 & 5 & 8 & $\overline{0}$ & 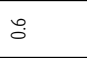 & 5 & 8 \\
\hline$>$ & 足 & $\begin{array}{l}\text { 响 } \\
\stackrel{\overbrace{}}{=}\end{array}$ & 商 & 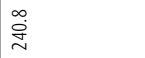 & 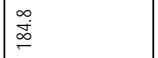 & $\stackrel{m}{\grave{d}}$ & 商 & $\begin{array}{l}\infty \\
\infty \\
\aleph_{0}^{\circ}\end{array}$ & 咅 & 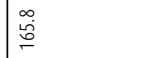 \\
\hline อ̃ & 8 & 苟 & $\stackrel{9}{\circ}$ & $\stackrel{9}{:}$ & 8 & $\stackrel{\infty}{\infty}$ & $\stackrel{\infty}{\infty}$ & 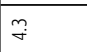 & 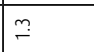 & $\stackrel{\circ}{\circ}$ \\
\hline : & 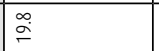 & $\stackrel{i}{i}$ & $m$ & 8 & $\approx$ & b. & 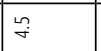 & $\bar{m}$ & \begin{tabular}{|l|}
$j$ \\
$m$
\end{tabular} & \begin{tabular}{|l|}
$\pi$ \\
\end{tabular} \\
\hline$\widehat{\circ}$ & $\stackrel{\circ}{\circ}$ & 4 & 8 & g. & $\infty$ & ?ח & g. & 8 & $\cong$ & \begin{tabular}{|l|}
$\infty$ \\
0 \\
0
\end{tabular} \\
\hline 을 & $\frac{9}{7}$ & $\begin{array}{ll}\infty \\
\stackrel{m}{m} \\
m\end{array}$ & 角 & 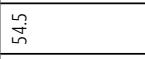 & 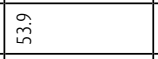 & 足 & 号 & $\begin{array}{l} \\
\end{array}$ & 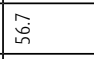 & in \\
\hline ๑n & ¿ें & $\stackrel{m}{g}$ & $\stackrel{\circ}{\circ}$ & $\stackrel{n}{\sim}$ & $\stackrel{\sim}{\pi}$ & 苞 & $\stackrel{\llcorner}{\stackrel{\infty}{\infty}}$ & $\stackrel{n}{m}$ & $\approx$ & $\stackrel{\rho}{\check{r}}$ \\
\hline 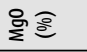 & $=$ & $\stackrel{i}{i}$ & $\stackrel{n}{m}$ & $\stackrel{\infty}{i}$ & $m$ & $\stackrel{P}{q}$ & $\bar{i}$ & $\exists$ & o & $\stackrel{\circ}{\circ}$ \\
\hline ז̃e & na & m & 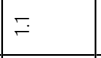 & 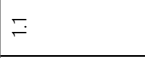 & $\stackrel{\circ}{\circ}$ & $\stackrel{\sim}{i}$ & $\simeq$ & 8 & 2 & $\infty$ \\
\hline$\infty$ & $\underset{d}{J}$ & $\stackrel{I}{I}$ & $\stackrel{\tilde{I}}{\stackrel{2}{I}}$ & $\underline{m}$ & $\stackrel{m}{g}$ & 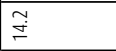 & $\stackrel{i}{i}$ & $\stackrel{.}{\circ}$ & 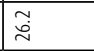 & 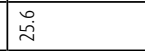 \\
\hline$=$ & $\bar{E}$ & $\bar{m}$ & 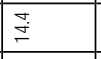 & \begin{tabular}{|l|}
$a$ \\
$\infty$
\end{tabular} & So & \begin{tabular}{|l|} 
\\
\end{tabular} & $\bar{\infty}$ & in & in & $\Phi$ \\
\hline 总高 & 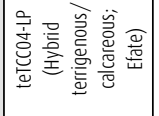 & 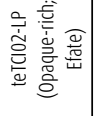 & 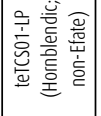 & 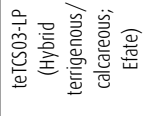 & 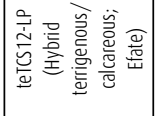 & 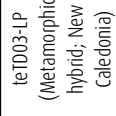 & $\mid$ & 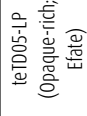 & 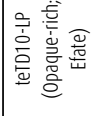 & 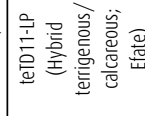 \\
\hline 关 & 总 & $\begin{array}{l}\text { 总 } \\
\text { 总 }\end{array}$ & $\mid$ & 总 & 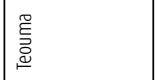 & $\begin{array}{l}\text { 黑 } \\
\text { 总 }\end{array}$ & 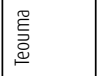 & $\begin{array}{l}\text { 志 } \\
\text { 噌 }\end{array}$ & \begin{tabular}{|l|} 
兽 \\
|
\end{tabular} & 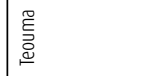 \\
\hline
\end{tabular}




\section{Conclusion}

This study provides a solid data set supporting the idea that important modifications occurred in pottery manufacturing behaviour between Lapita and immediately Post-Lapita phases in Vanuatu. The cessation of the production of dentate-stamped pottery coincided with a modification of the conceptualisation of pottery production as raw material procurement patterns were completely modified. While multiple temper types and a wide compositional variability were identified in decorated Lapita assemblages, immediately Post-Lapita ceramics are predominantly manufactured from a limited range of local raw materials. Given that the decisions related to pottery manufacture were taken based on underlying social values, the resulting variety of technological styles observable in decorated Lapita ceramic assemblages inform about the social aspects of these groups. It is suggested that no political control over the raw materials was exerted within the Lapita groups examined, nor that pottery production was specialised. Rather there was little sociopolitical differentiation among the members of the community in the early phases of Lapita occupation.

The homogenisation of the raw materials in parallel with increasing regionalisation of decoration support the idea of major societal changes. The contrast between Lapita variability and PostLapita homogeneity is so vivid that it is interpreted as a statement marking social difference. The synchronised transformation of compositional, decorative and morphological aspects of Lapita pottery indicates that long-established customary ways of manufacturing pottery were no longer relevant in Vanuatu by c. 2800 BP.

\section{Acknowledgements}

The research and fieldwork were funded by an Australian National University Research Scholarship for International Students and a Doctorate scholarship from the Fonds de Recherche du Québec-Société et Culture. I would also like to acknowledge the support of the College of Asia and the Pacific at The Australian National University for hosting me as a Visiting Fellow during the writing of this chapter. All figures and tables were produced by the author.

\section{References}

Aide, M.T. and C. Aide 2012. Rare earth elements: Their importance in understanding soil genesis. ISRN Soil Science 2012. ID 783876. doi.org/10.5402/2012/783876.

Ambrose, W. 1992. Clays and sands in Melanesian pottery analysis. In J.-C. Galipaud (ed.), Poterie Lapita et peuplement, pp. 169-176. ORSTOM, Nouméa.

Ambrose, W. 1997. Contradictions in Lapita pottery, a composite clone. Antiquity 71:525-538. doi. org/10.1017/S0003598X00085306.

Ambrose, W. 2007. The implements of Lapita ceramic stamped ornamentation. In S. Bedford, C. Sand and S.P. Connaughton (eds), Oceanic explorations: Lapita and Western Pacific settlement, pp. 213-221. Terra Australis 26. ANU E Press, Canberra. doi.org/10.22459/TA26.2007.

Anson, D. 1999. Compositional analyses of dentate-stamped Lapita and nail-incised and applied relief pottery from Watom Island. In J.-C. Galipaud and I. Lilley (eds), The Pacific from 5000 to 2000 BP: Colonisation and transformations, pp. 85-102. IRD Éditions, Paris.

Arnold, D.E. 1992. Commentary on Section II. In H. Neff (ed.), Chemical characterization of ceramic pastes in archaeology, pp. 159-166. Prehistory Press, Madison, WI. 
Arnold, D.E., H. Neff, R.L. Bishop and M.D. Glascock 1999. Testing interpretive assumptions of neutron activation analysis: Contemporary pottery in Yucatán. In E. Chilton (ed.), Material meanings: Critical approaches to the analysis of style, pp. 61-84. University of Utah Press, Salt Lake City.

Bedford, S. 2003. The timing and nature of Lapita colonisation in Vanuatu: The haze begins to clear. In C. Sand (ed.), Pacific archaeology: Assessments and prospects. Proceedings of the conference for the 50th anniversary of the first Lapita excavation, Kone-Nouméa, 2002, pp. 147-158. Les cahiers de l'archéologie en Nouvelle-Calédonie 15. Département Archéologie, Service des Musées et du Patrimoine de Nouvelle-Calédonie, Nouméa.

Bedford, S. 2006a. The Pacific's earliest painted pottery: An added layer of intrigue to the Lapita debate and beyond. Antiquity 80:544-557. doi.org/10.1017/S0003598X00094023.

Bedford, S. 2006b. Pieces of the Vanuatu puzzle: Archaeology of the north, south and centre. Terra Australis 23. Pandanus Books, The Australian National University, Canberra. doi.org/10.22459/ PVP.02.2007.

Bedford, S. 2007. Crucial first steps into Remote Oceania: Lapita in the Vanuatu Archipelago. In S. Chiu and C. Sand (eds), From Southeast Asia to the Pacific: Archaeological perspectives on the Austronesian expansion and the Lapita Cultural Complex, pp. 185-213. Centre for Archaeological Studies, Research Centre of Humanities and Social Sciences. Academia Sinica, Taipei.

Bedford, S. and G. Clark 2001. The rise and rise of the incised and applied relief tradition: A review and assessment. In G.R. Clark, A.J. Anderson and T. Vunidilo (eds), The archaeology of Lapita dispersal in Oceania. Papers from the Fourth Lapita Conference, June 2000, Canberra, Australia, pp. 61-74. Terra Australis 17. Pandanus Books, The Australian National University, Canberra.

Bedford, S. and J.-C. Galipaud 2010. Chain of islands: Lapita in the north of Vanuatu. In C. Sand and S. Bedford (eds), Lapita: Ancêtres Océaniens/Oceanic ancestors, pp. 123-137. Musée du quai Branly and Somogy, Paris.

Bedford, S. and M. Spriggs 2000. Crossing the Pwanmwou: Preliminary report on recent excavations adjacent to and south west of Mangaasi, Efate, Vanuatu. Archaeology in Oceania 35:120-126. doi. org/10.1002/j.1834-4453.2000.tb00465.x.

Bedford, S. and M. Spriggs 2007. Birds on the rim: A unique Lapita carinated vessel in its wider context. Archaeology in Oceania 42(1):12-21. doi.org/10.1002/j.1834-4453.2007.tb00010.x.

Bedford, S., M. Spriggs and R. Regenvanu 2006. The Teouma Lapita site and the early human settlement of the Pacific Islands. Antiquity 80(310):812-828. doi.org/10.1017/S0003598X00094448.

Bedford, S., M. Spriggs, R. Regenvanu, C. Macgregor, T. Kuautonga and M. Sietz 2007. The excavation, conservation and reconstruction of Lapita burial pots from the Teouma site, Efate, Central Vanuatu. In S. Bedford, C. Sand and S.P. Connaughton (eds), Oceanic explorations: Lapita and Western Pacific settlement, pp. 223-240. Terra Australis 26. ANU E Press, Canberra. doi.org/10.22459/TA26.2007.

Bedford, S., M. Spriggs, H.R. Buckley, F. Valentin, R. Regenvanu and M. Abong 2010. A cemetery of first settlement: The site of Teouma, South Efate, Vanuatu. In C. Sand and S. Bedford (eds), Lapita: Ancêtres Océaniens/Oceanic ancestors, pp. 140-161. Musée du quai Branly and Somogy, Paris.

Bedford, S., H.R. Buckley, F. Valentin, N.G. Tayles and N.F. Longga 2011. Lapita burials, a new Lapita cemetery and Post-Lapita burials from Malakula, northern Vanuatu, Southwest Pacific. Journal of Pacific Archaeology 2(2):26-48.

Best, S. 2002. Lapita: A view from the east. New Zealand Archaeological Association Monograph 24. New Zealand Archaeological Association, Auckland. 
Buxeda i Garrigós, J., H. Mommsen and A. Tsolakidou 2002. Alterations of $\mathrm{Na}, \mathrm{K}$ and $\mathrm{Rb}$ concentrations in Mycenaean pottery and a proposed explanation using X-Ray diffraction. Archaeometry 44(2):187-198. doi.org/10.1111/1475-4754.t01-1-00052.

Chiu, S. 2003a. Social and economic meanings of Lapita pottery: A New Caledonian case. In C. Sand (ed.), Pacific archaeology: Assessments and prospects. Proceedings of the conference for the 50th anniversary of the first Lapita excavation, Kone-Nouméa, 2002, pp. 159-182. Les cahiers de l'archéologie en Nouvelle-Calédonie 15. Département Archéologie, Service des Musées et du Patrimoine de NouvelleCalédonie, Nouméa.

Chiu, S. 2003b. The socio-economic functions of Lapita ceramic production and exchange: A case study from site WKO013A, Koné, New Caledonia. Unpublished PhD thesis, University of California, Berkeley.

Chiu, S. 2005. Meanings of a Lapita face: Materialized social memory in ancient house societies. Taiwan Journal of Anthropology 3(1):1-47.

Chiu, S. 2007. Detailed analysis of Lapita face motifs: Case studies from Reef/Santa Cruz Lapita sites and New Caledonia Lapita site 13A. In S. Bedford, C. Sand and S.P. Connaughton (eds), Oceanic explorations: Lapita and Western Pacific settlement, pp. 241-264. Terra Australis 26. ANU E Press, Canberra. doi.org/10.22459/TA26.2007.

Clark, G. 2007. Specialisation, standardisation and Lapita ceramics. In S. Bedford, C. Sand and S.P. Connaughton (eds), Oceanic explorations: Lapita and Western Pacific settlement, pp. 289-299. Terra Australis 26. ANU E Press, Canberra. doi.org/10.22459/TA26.2007.

Conkey, M.W. 1978. Style and information in cultural evolution: Toward a predictive model for the Paleolithic. In C.L. Redman, M.J. Berman, E.V. Curtin, W.T. Langhorne, N.M. Versaggi and J.C. Wanser (eds), Social archaeology: Beyond subsistence and dating, pp. 61-85. Academic Press, New York.

Constantine, A., C. Reepmeyer, S. Bedford, M. Spriggs and M. Ravn 2015. Obsidian distribution from a Lapita cemetery sheds light on its value to past societies. Archaeology in Oceania 50(2):111-116. doi.org/10.1002/arco.5064.

Costin, C.L. and M.B. Hagstrum 1995. Standardization, labour investment, skill, and the organization of ceramic production in Late Prehispanic Highland Peru. American Antiquity 60(4):619-639. doi. org/10.2307/282046.

Dickinson, W.R. 1995. Petrographic report WRD-117. Temper types in prehistoric Vanuatu potsherds indigenous to Efate, Santo, Malekula, and other islands of the New Hebrides Island Arc. Unpublished report.

Dickinson, W.R. 2003. Petrographic report WRD-228. Petrography of temper sands in sherds from Vao in Vanuatu. Unpublished report.

Dickinson, W.R. 2006a. Temper sands in prehistoric Oceanian pottery: Geotectonics, sedimentology, petrography, provenance. Geological Society of America, Special Paper 406. Geological Society of America, Boulder, Colorado. doi.org/10.1130/2006.2406.

Dickinson, W.R. 2006b. Petrographic report WRD-138. Petrography of deep sherds and stream sediment from Efate, Vanuatu. In S. Bedford, Pieces of the Vanuatu puzzle: Archaeology of the north, south and centre, pp. 311. Terra Australis 23. Pandanus Books, The Australian National University, Canberra. doi.org/10.22459/PVP.02.2007.

Dickinson, W.R. 2006c. Petrographic report WRD-180. Petrography of sand tempers in selected Late-style sherds from Malekula in Vanuatu and comparison with other Malekula sherds. In S. Bedford, Pieces of the Vanuatu puzzle: Archaeology of the north, south and centre, pp. 313-315. Terra Australis 23. Pandanus Books, The Australian National University, Canberra. doi.org/10.22459/PVP.02.2007. 
Dickinson, W.R. 2006d. Petrographic report WRD-184. Petrography of two unusual sherds from Erromango and Malekula. In S. Bedford, Pieces of the Vanuatu puzzle: Archaeology of the north, south and centre, pp. 316-317. Terra Australis 23. Pandanus Books, The Australian National University, Canberra. doi.org/10.22459/PVP.02.2007.

Dickinson, W.R., R. Shutler, R. Shortland, D.V. Burley and T.S. Dye 1996. Sand tempers in indigenous Lapita and Lapitoid Polynesian plainware and imported protohistoric Fijian pottery of Haapai (Tonga) and the question of Lapita tradeware. Archaeology in Oceania 31:87-98. doi. org/10.1002/j.1834-4453.1996.tb00351.x.

Dickinson, W.R., S. Bedford and M. Spriggs 2013. Petrography of temper sands in 112 reconstructed Lapita pottery vessels from Teouma (Efate): Archaeological implications and relations to other Vanuatu tempers. Journal of Pacific Archaeology 4(2):1-20.

Dobres, M.-A. and C.R. Hoffman 1994. Social agency and the dynamics of prehistoric technology. Journal of Archaeological Method and Theory 1(3):211-258. doi.org/10.1007/BF02231876.

Earle, T. and M. Spriggs 2015. Political economy in prehistory. A Marxist approach to Pacific sequences. Current Anthropology 56(4):515-544. doi.org/10.1086/682284.

Gaffney, D., G.R. Summerhayes, A. Ford, J.M. Scott, T. Denham, J. Field and W.R. Dickinson 2015. Earliest pottery on New Guinea mainland reveals Austronesian influences in highland environments 3000 years ago. PLoS ONE 10(9):e0134497. doi.org/10.1371/journal.pone.0134497.

Galipaud, J.-C. 1990. The physico-chemical analysis of ancient pottery from New Caledonia. In M. Spriggs (ed.), Lapita design, form and composition. Proceedings of the Lapita Design Workshop, Canberra, December 1988, pp. 134-142. Department of Prehistory, RSPacS, The Australian National University, Canberra.

Garanger, J. 1971. Incised and applied relief pottery, its chronology and development in southeastern Melanesia, and extra areal comparisons. In R.C. Green and M. Kelly (eds), Studies in Oceanic culture history, Volume 2, pp. 53-66. Pacific Anthropological Records 12. Department of Anthropology, Bernice P. Bishop Museum, Honolulu.

Golitko, M., J.V. Dudgeon, H. Neff and J.E. Terrell 2012. Identification of post-depositional chemical alteration of ceramics from the North Coast of Papua New Guinea (Sanduan Province) by timeof-flight-laser-ablation-inductively coupled plasma-mass-spectrometry (TOF-LA-ICP-MS). Archaeometry 54(1):80-100. doi.org/10.1111/j.1475-4754.2011.00612.x.

Gratuze, B. 1999. Obsidian characterization by laser ablation ICP-MS and its application to prehistoric trade in the Mediterranean and the Near East: Sources and distribution of obsidian within the Aegean and Anatolia. Journal of Archaeological Science 26:869-881. doi.org/10.1006/jasc.1999.0459.

Gratuze, B., M. Blet-Lemarquand and J.-N. Barrandon 2001. Mass spectrometry with laser sampling: A new tool to characterize archaeological materials. Journal of Radioanalytical and Nuclear Chemistry 247(3):645-656. doi.org/10.1023/A:1010623703423.

Green, R.C. 1987. Obsidian results from the Lapita sites of the Reef/Santa Cruz Islands. In W.R. Ambrose and J.M.J. Mummery (eds), Archaeometry: Further Australian studies, pp. 239-249. Occasional Papers in Prehistory 14, Department of Prehistory, RSPacS, The Australian National University, Canberra.

Green, R.C. 1991. The Lapita Cultural Complex: Current evidence and proposed models. Bulletin of the Indo-Pacific Prehistory Association 11:296-305. doi.org/10.7152/bippa.v11i0.11393.

Green, R.C. 2000. Lapita and the cultural model for intrusion, integration and innovation. In A.J. Anderson and T. Murray (eds), Australian archaeologist: Collected papers in honour of Jim Allen, pp. 372-392. Coombs Academic Publishing, The Australian National University, Canberra. 
Green, R.C. and D. Anson 1991. The Reber-Rakival Lapita site on Watom. Implications of the 1985 excavations at the SAC and SDI localities. In J. Allen and C. Gosden (eds), Report of the Lapita Homeland Project, pp. 170-181. Department of Prehistory, RSPacS, The Australian National University, Canberra.

Green, R.C. and P.V. Kirch 1997. Lapita exchange systems and their Polynesian transformations: Seeking explanatory models. In M.I. Weisler (ed.), Prehistoric long-distance interaction in Oceania: An interdisciplinary approach, pp. 19-37. New Zealand Archaeological Association Monograph 21. New Zealand Archaeological Association, Auckland.

Harding, S. and R. Payne 2011. A guide to multivariate analysis in GenStat ${ }^{\circ}$. VSN International, Hemel Hempstead.

Hegmon, M. 1998. Technology, style, and social practices: Archaeological approaches. In M.T. Stark (ed.), The archaeology of social boundaries, pp. 264-280. Smithsonian Institution Press, Washington DC.

Hunt, T.L. 1989. Lapita ceramic exchange in the Mussau Islands, Papua New Guinea. Unpublished PhD thesis, University of Washington, Seattle.

Kirch, P.V. 1988. Long-distance exchange and island colonization: The Lapita case. Norwegian Archaeological Review 21(2):103-117. doi.org/10.1080/00293652.1988.9965475.

Kirch, P.V. 1991. Prehistoric exchange in Western Melanesia. Annual Review of Anthropology 20:141165. doi.org/10.1146/annurev.an.20.100191.001041.

Kirch, P.V. 1997. The Lapita peoples: Ancestors of the Oceanic world. Blackwell, Cambridge.

Kirch, P.V. 2000. On the road of the winds: An archaeological history of the Pacific Islands before European contact. University of California Press, Berkeley.

Kirch, P.V., T.L. Hunt, M.I. Weisler, V.L. Butler and M.S. Allen 1991. Mussau Islands prehistory: Results of the 1985-86 excavations. In J. Allen and C. Gosden (eds), Report of the Lapita Homeland Project, pp. 144-163. Occasional papers in Prehistory 20. Department of Prehistory, RSPacS, The Australian National University, Canberra.

Lechtman, H. 1977. Style in technology—Some early thoughts. In H. Lechtman and R.S. Merrill (eds), Material culture: styles, organization and dynamics of technology, pp. 3-20. West, St Paul, MN.

Leclerc, M. 2016. Investigating the raw materials used for Lapita and Post-Lapita pottery manufacturing: A chemical characterisation of ceramic collections from Vanuatu. Unpublished $\mathrm{PhD}$ thesis, The Australian National University, Canberra.

Leclerc, M., K. Taché, S. Bedford, M. Spriggs, A. Lucquin and O.E. Craig 2018. Organic residue analysis documents the peculiar use of Lapita dentate-stamped vessels. Journal of Archaeological Science: Reports 17:712-722.

Leclerc, M., E. Grono, S. Bedford and M. Spriggs 2019. Assessment of the technological variability in decorated Lapita pottery from Teouma, Vanuatu, by petrography and LA-ICP-MS: Implications for Lapita social organisation. Archaeological and Anthropological Sciences 2019:1-17. doi.org/10.1007/ s12520-019-00862-z.

Lemonnier, P. 1993. Introduction. In P. Lemonnier (ed.), Technological choices: Transformation in material cultures since the Neolithic, pp. 1-35. Routledge, London. doi.org/10.4324/9781315887630.

Marshall, Y. 1985. Who made the Lapita pots? A case study in gender archaeology. Journal of the Polynesian Society 94(3):205-233. 
Mead, S.M. 1975. The decorative system of the Lapita potters of Sigatoka, Fiji. In S.M. Mead, L. Birks, H. Birks and E. Shaw (eds), The Lapita pottery style of Fiji and its associations, pp. 19-43. The Polynesian Society Memoir No. 38. Polynesian Society, Wellington.

Mitchell, A.H.G. 1966. Geology of South Malekula. New Hebrides Condominium Geological Survey Regional Report, Port Vila.

Mitchell, A.H.G. 1971. Geology of Northern Malekula. New Hebrides Condominium Geological Survey Regional Report, Port Vila.

Neff, H. 2003. Analysis of Mesoamerican plumbate pottery surfaces by laser ablation-inductively coupled plasma-mass spectrometry (LA-ICP-MS). Journal of Archaeological Science 30:21-35. doi. org/10.1006/jasc.2001.0801.

Nunn, P.D. and F. Petchey 2013. Bayesian re-evaluation of Lapita settlement in Fiji: Radiocarbon analysis of the Lapita occupation at Bourewa and nearby sites on the Rove Peninsula, Viti Levu Island. Journal of Pacific Archaeology 4(2):21-34.

Peacock, D.P.S. 1970. The scientific analysis of ancient ceramics: A review. World Archaeology 1(3):375389. doi.org/10.1080/00438243.1970.9979454.

Petchey, F., M. Spriggs, S. Bedford, F. Valentin and H.R. Buckley 2014. Radiocarbon dating of burials from the Teouma Lapita cemetery, Efate, Vanuatu. Journal of Archaeological Science 50:227-242. doi. org/10.1016/j.jas.2014.07.002.

Petchey, F., M. Spriggs, S. Bedford and F. Valentin 2015. The chronology of occupation at Teouma, Vanuatu: Use of a modified chronometric hygiene protocol and Bayesian modeling to evaluate midden remains. Journal of Archaeological Science: Reports 4:95-105. doi.org/10.1016/j. jasrep.2015.08.024.

Picon, M. 1991. Quelques observations complémentaires sur les altérations de la composition chimique des céramiques au cours du temps. Revue d'Archéométrie 15:117-122. doi.org/10.3406/ arsci.1991.1263.

Reepmeyer, C. 2008. Characterising volcanic glass sources in the Banks Islands, Vanuatu. Archaeology in Oceania 43:120-127. doi.org/10.1002/j.1834-4453.2009.tb00038.x.

Reepmeyer, C., M. Spriggs, S. Bedford and W. Ambrose 2010. Provenance and technology of lithic artifacts from the Teouma Lapita site, Vanuatu. Asian Perspectives 49(1):205-225. doi.org/10.1353/ asi.2010.0004.

Reepmeyer, C., M. Spriggs, Anggraeni, P. Lape, L. Neri, W.P. Ronquillo, T. Simanjuntak, G.R. Summerhayes, D. Tanudirjo and A. Tiauzon 2011. Obsidian sources and distribution systems in Island Southeast Asia: New results and implications from geochemical research using LA-ICPMS. Journal of Archaeological Science 38:2995-3005. doi.org/10.1016/j.jas.2011.06.023.

Rice, P.M. 1991. Specialisation, standardisation, and diversity: A retrospective. In R.L. Bishop and F.W. Lange (eds), The ceramic legacy of Anna O. Shepard, pp. 257-279. University Press of Colorado, Boulder.

Rye, O.S. 1976. Keeping your temper under control: Materials and the manufacture of Papuan pottery. Archaeology and Physical Anthropology in Oceania 11(2):106-137.

Sand, C. 2007. Looking at the big motifs: A typology of the central band decorations of the Lapita ceramic tradition of New Caledonia (Southern Melanesia) and preliminary regional comparisons. In S. Bedford, C. Sand and S.P. Connaughton (eds), Oceanic explorations: Lapita and Western Pacific settlement, pp. 265-287. Terra Australis 26. ANU E Press, Canberra. doi.org/10.22459/TA26.2007. 
Sand, C. 2010. Lapita Calédonien. Archéologie d'un premier peuplement insulaire Océanien. Société des Océanistes, Paris. doi.org/10.4000/books.sdo.1128.

Sand, C. and S. Bedford 2010. Lapita: Ancêtres Océaniens/Oceanic ancestors. Musée du quai Branly and Somogy, Paris.

Sand, C., J. Bolé and A.-J. Ouetcho 2011. A revision of New Caledonia's ceramic sequence. Journal of Pacific Archaeology 2(1):56-68.

SAS Institute Inc. 2015. JMP 12 Multivariate Methods. SAS Institute Inc., Cary, NC.

Sheppard, P.J. 1993. Lapita lithics: Trade/exchange and technology. A view from the Reefs/Santa Cruz. Archaeology in Oceania 28(3):121-137. doi.org/10.1002/j.1834-4453.1993.tb00303.x.

Sheppard, P.J. 2011. Lapita colonization across the Near/Remote Oceania boundary. Current Anthropology 52(6):799-840. doi.org/10.1086/662201.

Sheppard, P.J., S. Chiu and R. Walter 2015. Re-dating Lapita movement into Remote Oceania. Journal of Pacific Archaeology 6(1):26-36.

Sillar, B. and M.S. Tite 2000. The challenge of 'technological choice' for materials science approaches in archaeology. Archaeometry 42(1):2-20. doi.org/10.1111/j.1475-4754.2000.tb00863.x.

Siorat, J.-P. 1990. A technological analysis of Lapita pottery decoration. In M. Spriggs (ed.), Lapita design, form and composition: Proceedings of the Lapita Design Workshop, Canberra, December 1988, pp. 59-82. Occasional Papers in Prehistory 19. Department of Prehistory, RSPacS, The Australian National University, Canberra.

Skelly, R., B. David, F. Petchey and M. Leavesley 2014. Tracking ancient beach-lines inland: 2600-yearold dentate-stamped ceramics at Hopo, Vailala River region, Papua New Guinea. Antiquity 88(340):470-487. doi.org/10.1017/S0003598X00101127.

Speakman, R.J. and H. Neff 2005. The application of laser ablation-ICP-MS to the study of archaeological materials-An introduction. In R.J. Speakman and H. Neff (eds), Laser ablation ICPMS in archaeological research, pp. 1-14. University of New Mexico Press, Albuquerque.

Specht, J., T. Denham, J. Goff and J.E. Terrell 2014. Deconstructing the Lapita Cultural Complex in the Bismarck Archipelago. Journal of Archaeological Research 22(2):89-140. doi.org/10.1007/s10814013-9070-4.

Spriggs, M. 1990. The changing face of Lapita: Transformation of a design. In M. Spriggs (ed.), Lapita design, form and composition: Proceedings of the Lapita Design Workshop, Canberra, December 1988, pp. 83-122. Occasional Papers in Prehistory 19. Department of Prehistory, RSPacS, The Australian National University, Canberra.

Spriggs, M. 2002. They've grown accustomed to your face. In S. Bedford, C. Sand and D. Burley (eds), Fifty years in the field: Essays in honour and celebration of Richard Shutler Jr's archaeological career, pp. 51-57. New Zealand Archaeological Association Monograph 25. New Zealand Archaeological Association, Auckland.

Spriggs, M. 2003. Post-Lapita evolutions in Island Melanesia. In C. Sand (ed.), Pacific archaeology: Assessments and prospects. Proceedings of the conference for the 50th anniversary of the first Lapita excavation, Kone-Nouméa, 2002, pp. 205-212. Les cahiers de l'archéologie en NouvelleCalédonie 15. Département Archéologie, Service des Musées et du Patrimoine de NouvelleCalédonie, Nouméa. 
Spriggs, M. and S. Bedford 2001. Arapus: A Lapita site at Mangaasi in central Vanuatu? In G.R. Clark, A.J. Anderson and T. Vunidilo (eds), The archaeology of Lapita dispersal in Oceania: Papers from the Fourth Lapita Conference, June 2000, Canberra, Australia, pp. 93-104. Terra Australis 17. Pandanus Books, The Australian National University, Canberra.

Spriggs, M. and S. Bedford 2013. Is there an incised Lapita phase after dentate-stamped pottery ends? Data from Teouma, Efate Island, Vanuatu. In G.R. Summerhayes and H. Buckley (eds), Pacific archaeology: Documenting the past 50,000 years, pp. 148-156. University of Otago Studies in Archaeology 25. University of Otago, Dunedin.

Summerhayes, G.R. 1997. Losing your temper: The effect of mineral inclusions on pottery analyses. Archaeology in Oceania 32(1):108-117. doi.org/10.1002/j.1834-4453.1997.tb00376.x.

Summerhayes, G.R. 2000a. Lapita interaction. Terra Australis 15. Department of Archaeology and Natural History and the Centre for Archaeological Research, The Australian National University, Canberra.

Summerhayes, G.R. 2000b. What's in a pot? In A.J. Anderson and T. Murray (eds), Australian archaeologist. Collected papers in honour of Jim Allen, pp. 291-307. Coombs Academic Publishing, The Australian National University, Canberra.

Summerhayes, G.R. and J. Allen. 2007. Lapita writ small? Revisiting the Austronesian colonisation of the Papuan South Coast. In S. Bedford, C. Sand and S.P. Connaughton (eds), Oceanic explorations: Lapita and Western Pacific settlement, pp. 97-122. ANU E Press, Canberra. doi.org/10.22459/ TA26.2007.

Terrell, J.E. and R.L. Welsch. 1997. Lapita and the temporal geography of prehistory. Antiquity 71:548572. doi.org/10.1017/S0003598X0008532X.

Tryon, D. 1996. Dialect chaining and the use of geographical space. In J. Bonnemaison, K. Huffman, C. Kaufmann and D. Tryon (eds), Arts of Vanuatu, pp. 170-181. Crawford House, Bathurst.

Valentin, F., E. Herrscher, S. Bedford, M. Spriggs and H.R. Buckley 2014. Evidence for social and cultural change in Central Vanuatu between 3000 and 2000 BP: Comparing funerary and dietary patterns of the first and later generations at Teouma, Efate. The Journal of Island and Coastal Archaeology 9(3):381-399. doi.org/10.1080/15564894.2014.921958.

Valentin, F., F. Détroit, M. Spriggs and S. Bedford 2016. Early Lapita skeletons from Vanuatu show Polynesian cranifacial shape: Implications for Remote Oceania settlement and Lapita origins. Proceedings from the National Academy of Sciences 113(2):292-297. doi.org/10.1073/ pnas. 1516186113.

van der Leeuw, S.E., D.A. Papousek and A. Coudart 1991. Technical traditions and unquestioned assumptions: The case of pottery in Michoacan. Techniques et Culture 17-18:145-173. doi. org/10.4000/tc.691.

White, A.W., T.H. Worthy, S. Hawkins, S. Bedford and M. Spriggs 2010. Megafaunal meiolaniid horned turtles survived until early human settlement in Vanuatu, Southwest Pacific. Proceedings from the National Academy of Sciences 107(35):15512-15516. doi.org/10.1073/pnas.1005780107.

Wobst, M.H. 1977. Stylistic behavior and information exchange. In C.E. Cleland (ed.), For the director: Research essays in honor of James B. Griffin, pp. 317-342. Anthropological Papers 61. Museum of Anthropology, University of Michigan, Ann Arbour.

Worthy, T.H., S. Hawkins, S. Bedford and M. Spriggs 2015. Avifauna from the Teouma Lapita site, Efate Island, Vanuatu, including a new genus and species of megapode. Pacific Science 69(2):205254. doi.org/10.2984/69.2.6. 
This text is taken from Debating Lapita: Distribution, Chronology, Society and Subsistence, edited by Stuart Bedford and Matthew Spriggs, published 2019 by ANU Press,

The Australian National University, Canberra, Australia.

doi.org/10.22459/TA52.2019.17 